\title{
Differential rotation in Sun-like stars from surface variability and asteroseismology
}

\author{
Dissertation \\ zur Erlangung des mathematisch-naturwissenschaftlichen Doktorgrades \\ "Doctor rerum naturalium" \\ der Georg-August-Universität Göttingen \\ im Promotionsprogramm PROPHYS \\ der Georg-August University School of Science (GAUSS)
}

vorgelegt von

Martin Bo Nielsen

aus Aarhus, Dänemark

Göttingen, 2016 
Betreuungsausschuss

\section{Prof. Dr. Laurent Gizon}

Institut für Astrophysik, Georg-August-Universität Göttingen

Max-Planck-Institut für Sonnensystemforschung, Göttingen, Germany

\section{Dr. Hannah Schunker}

Max-Planck-Institut für Sonnensystemforschung, Göttingen, Germany

\section{Prof. Dr. Ansgar Reiners}

Institut für Astrophysik, Georg-August-Universität, Göttingen, Germany

$\underline{\text { Mitglieder der Prüfungskommision }}$

Referent: Prof. Dr. Laurent Gizon

Institut für Astrophysik, Georg-August-Universität Göttingen

Max-Planck-Institut für Sonnensystemforschung, Göttingen

Korreferent: Prof. Dr. Stefan Dreizler

Institut für Astrophysik, Georg-August-Universität, Göttingen

2. Korreferent: Prof. Dr. William Chaplin

School of Physics and Astronomy, University of Birmingham

Weitere Mitglieder der Prüfungskommission:

Prof. Dr. Jens Niemeyer

Institut für Astrophysik, Georg-August-Universität, Göttingen

PD. Dr. Olga Shishkina

Max Planck Institute for Dynamics and Self-Organization

Prof. Dr. Ansgar Reiners

Institut für Astrophysik, Georg-August-Universität, Göttingen

Prof. Dr. Andreas Tilgner

Institut für Geophysik, Georg-August-Universität, Göttingen 
Tag der mündlichen Prüfung: 



\section{Contents}

1 Introduction $\quad 11$

1.1 Evolution of stellar rotation rates . . . . . . . . . . . . . . . 11

1.1.1 Rotation on the pre-main-sequence . . . . . . . . . . . 11

1.1.2 Main-sequence rotation . . . . . . . . . . . . . . . . 13

1.1.2.1 Solar rotation . . . . . . . . . . . 15

1.1.3 Differential rotation in other stars . . . . . . . . . . . 17

1.1.3.1 Latitudinal differential rotation . . . . . . . . . . 17

1.1.3.2 Radial differential rotation . . . . . . . . . . . . . 18

1.2 Measuring stellar rotation with Kepler . . . . . . . . . . . . . . . . . 19

1.2.1 Kepler photometry . . . . . . . . . . . . . . . . . 19

1.2.2 Photometric time series analysis . . . . . . . . . . 20 20

1.2.2.1 The Lomb-Scargle periodogram . . . . . . . . . . . . 20

1.2.3 Measuring rotation from surface variability . . . . . . . . . 22

1.2.3.1 Measuring differential rotation with surface variability . 23

1.2.4 Measuring rotation from asteroseismology . . . . . . . . . 23

1.2.4.1 Measuring differential rotation with asteroseismology . 24

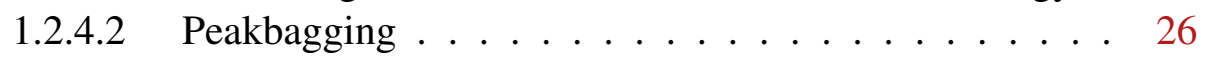

1.3 Applications of rotation measurements . . . . . . . . . . . . . . 34

1.3.1 Gyrochronology . . . . . . . . . . . . . . . . 34

1.3.2 Impact of rotation on stellar evolution . . . . . . . . . . . 36

1.3.3 Solar and stellar dynamos . . . . . . . . . . . . 36

2 Paper I - Rotation periods of 12000 main-sequence Kepler stars 39

2.1 Summary of Paper I . . . . . . . . . . . . . . . . . . . . . . . 39

2.2 Introduction . . . . . . . . . . . . . . . . . . 39

2.3 Measuring stellar rotation . . . . . . . . . . . . . . . . . 40

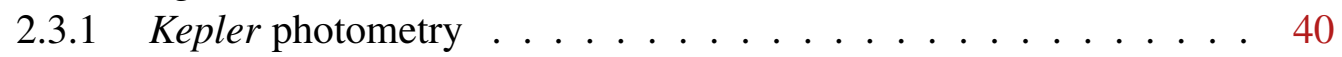

2.3.2 Detecting rotation periods ................ 40

2.3.3 Selecting stable rotation periods . . . . . . . . . . . . . 41

2.4 Consistency with $v \sin i$ measurements . . . . . . . . . . . . . . . . . . 43

2.5 Rotation of late type stars . . . . . . . . . . . . . . . . 45

2.6 Conclusions . . . . . . . . . . . . . . . . . 46

3 Paper II - Rotational splitting as a function of mode frequency for six Sun$\begin{array}{ll}\text { like stars } & 49\end{array}$

3.1 Summary of Paper II . . . . . . . . . . . . . . . . . . . . . . . . 49 
3.2 Introduction . . . . . . . . . . . . . . . . . . . 49

3.3 Analysis . . . . . . . . . . . . . . . . 50

3.3.1 Observations ........................ 51

3.3 .2 Power spectrum model . . . . . . . . . . . . . . . 51

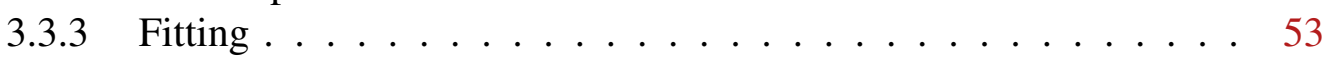

3.4 Rotation and inclination as a function of frequency $\ldots \ldots \ldots 5$

4 Paper III - Constraining differential rotation of Sun-like stars from astero$\begin{array}{lr}\text { seismic and starspot rotation periods } & 59\end{array}$

4.1 Summary of Paper III . . . . . . . . . . . . . . . . . . . . . . . 59

4.2 Introduction . . . . . . . . . . . . . . . . . . . 59

4.3 Measuring rotation periods . . . . . . . . . . . . . . . 60

4.3.1 Asteroseismic rotation periods . . . . . . . . . . . . 60

4.3.2 Surface variability periods . . . . . . . . . . . . . 62

4.4 Comparing asteroseismic and surface variability periods . . . . . . . . 64

4.5 Gyrochronology . . . . . . . . . . . . . . . . . 67

4.6 Conclusions . . . . . . . . . . . . . . . . . . . 68

5 Discussion: constraining interior rotational shear 71

5.1 Modeling radial differential rotation . . . . . . . . . . . . . 71

5.1.1 Computing the mode set splittings . . . . . . . . . . . . 72

5.1.2 Using only seismic data to constrain the radial shear . . . . . . . 74

5.1.3 Using prior information from surface variability . . . . . . . . 76

5.2 Conclusion . . . . . . . . . . . . . . . . 81

$\begin{array}{ll}\text { Bibliography } & 83\end{array}$

$\begin{array}{ll}\text { A Clusters used in Fig. 1.1 } & 103\end{array}$

B Detrending and corrections in PDC/msMAP data 105

$\begin{array}{ll}\text { C Measuring rotation with spectroscopy } & 107\end{array}$

$\begin{array}{lr}\text { Publications } & 109\end{array}$

$\begin{array}{ll}\text { Acknowledgements } & 111\end{array}$

$\begin{array}{lr}\text { Curriculum vitae } & 113\end{array}$ 


\section{Summary}

Thanks to helioseismology we know the internal rotation of the Sun as a function of radius and latitude. The presence of a shear at the base of the solar convection zone is thought to play a key role in the generation of magnetic fields. So far however, the internal rotation profiles of stars other than the Sun are unknown, and placing constraints on models of rotation and magnetic dynamos is therefore difficult. The NASA Kepler mission has provided high-quality photometric data that can be used to study the rotation of stars with two different techniques; asteroseismology and surface activity.

First, we developed an automated method for measuring the rotation of stars using surface variability. This was initially applied to $\sim 12000$ stars across the main sequence in the Kepler field, providing robust estimates of the surface rotation rates and the associated errors. We compared these measurements to spectroscopic $v \sin i$ values and found good agreement for F-,G- and K-type stars.

Second, we performed an asteroseismic analysis of six Sun-like stars, where we were able to measure the rotational splitting as a function of frequency in the $p$-mode envelope. The measured splittings were all consistent with a constant value, indicating little differential rotation.

Third, we compared the asteroseismic rotation rates of five Sun-like stars to their surface rotation rates. We found that the values were in good agreement, indicating little differential rotation between the regions where the two methods are most sensitive.

Finally, we discuss how the surface rotation rates may be used as a prior on the seismic envelope rotation rate, allowing us to find upper limits on the radial differential rotation in Sun-like stars. We find that the rotation rates of the radiative interior and convective envelope likely do not differ by more than $\sim 50 \%$. 



\section{Zusammenfassung}

Dank Helioseismologie kennen wir die innere Rotation der Sonne als Funktion des Radius und des Breitengrades. Es wird angenommen, dass die Anwesenheit einer Scherung an der Basis der Konvektionszone der Sonne eine wichtige Rolle bei der Erzeugung von Magnetfeldern spielt. Bisher allerdings sind die inneren Rotationsprofile von anderen Sternen als der Sonne unbekannt. Daher ist es schwierig, Modelle der Rotation und des magnetischen Dynamos einzugrenzen. Die Kepler Mission der NASA hat qualitativ hochwertige photometrische Daten bereitgestellt, die benutzt werden können um die Rotation von Sternen mit zwei verschiedenen Methoden zu erforschen: Asteroseismologie und Oberflächenaktivität.

Zuerst haben wir eine automatische Methode entwickelt um die Rotation von Sternen über ihre Oberflächenvariabilität zu messen. Diese Methode wurde zunächst auf 12 000 Sterne entlang der Hauptreihe im Kepler Feld angewandt. Dies ermöglichte zuverlässige Abschätzungen der Oberflächenrotationen und der dazugehörigen Fehler. Wir haben diese Messungen mit durch Spektroskopie bestimmten $v \sin i$ Werten verglichen und dabei gute übereinstimmungen für Sterne der Spektraltypen F,G und K gefunden.

Als zweites haben wir sechs sonnenähnliche Sternen mit Asteroseismologie analysiert. Hier konnten wir die Aufspaltung der Moden aufgrund der Rotation als Funktion der Frequenz für $p$-Moden messen. Die gemessenen Aufspaltungen waren alle konsistent mit einem konstanten Wert, was auf nur geringe differentielle Rotation hindeutet.

Als drittes haben wir für fünf sonnenähnliche Sterne die mittels Asteroseismologie bestimmten Rotationsraten mit den Oberflächenrotationsraten verglichen. Wir haben herausgefunden, dass die Werte gut übereinstimmen, was auf eine nur geringe differentielle Rotation zwischen den Bereichen hindeutet, wo die beiden Methoden am empfindlichsten sind.

Schlies̈lich diskutieren wir, wie die Oberflächenrotationsraten als erste Näherung für die mit Asteroseismologie bestimmten Rotationsraten der konvektiven Hülle verwendet werden können. Dies ermöglicht es, eine obere Grenze für die radiale differentielle Rotation in sonnenähnlichen Sternen zu finden. Unseren Ergebnissen zur Folge unterscheiden sich die Rotationsraten der Strahlungszone und der konvektiven Hülle wahrscheinlich um nicht mehr als $\sim 50 \%$ voneinander. 



\section{Introduction}

\subsection{Evolution of stellar rotation rates}

Stars rotate, but the transport of angular momentum (AM) has been a long standing problem for the study of rotation in stars. The rotation rate of a star can vary on scales of 0.1 - 100 days over the course of their evolution, but the open questions are still how and why it changes. This section is a brief overview of the rotation of stars on: the premain-sequence (PMS); the main sequence (MS), focusing on the detailed picture of solar rotation; and finally on what is currently known about radial and latitudinal differential rotation in other stars. This last section also covers rotation on the post-main-sequence as these are some of the few stars for which we have clear signs of radial differential rotation.

\subsubsection{Rotation on the pre-main-sequence}

The rotation of a star on the PMS sets the stage for all further evolution of its rotation rate on the MS and beyond. The exact nature however, of the AM evolution during these very early phases of stellar evolution is still poorly understood. This is in part because the only available indicator of the AM of a star is its surface rotation rate. For PMS stars this is measured by either spectroscopic $v \sin i$ (Vogel and Kuhi 1981, Hartmann et al. 1986, see also Appendix C) or observation of photometric variability from surface features (Rydgren and Vrba 1983, Herbst et al. 1987, see also Sect. 1.2.3). Many PMS stars appear to have strong surface magnetic fields, observed as spots on the stellar surface which can cover up to $\sim 30 \%$ of the star. They are generally considered fast rotators, making such observations relatively easy compared to older stars like the Sun. However, during the very early stages of the PMS evolution the star is shrouded in gas and dust, making direct probes of rotation very difficult (Herbst et al. 2007). The circumstellar material will eventually either be accreted onto the star, expelled by stellar winds and photon pressure, or form a disk, at which point the central star becomes visible.

Figure 1.1 shows the general trends of rotation as a function of age for a selection of open clusters stars available in the literature. The sequence starts with the very young Orion Nebula Cluster on the PMS, moving toward the zero-age-main sequence (ZAMS), and then onto the MS where the Sun is located. The ZAMS age for stars with masses in the range $0.5-1.5 \mathrm{M}_{\odot}$ is shown as the shaded region. For the majority of cluster stars (cool, low-mass stars) this point is reached somewhere between 100 - $200 \mathrm{Myr}$ after they initially reach hydrostatic quasi-equilibrium at the base of the Hayashi track.

During the PMS a star undergoes gravitational contraction, its moment of inertia decreases, and so from basic principles one expects the rotation rate to increase. From Fig. 


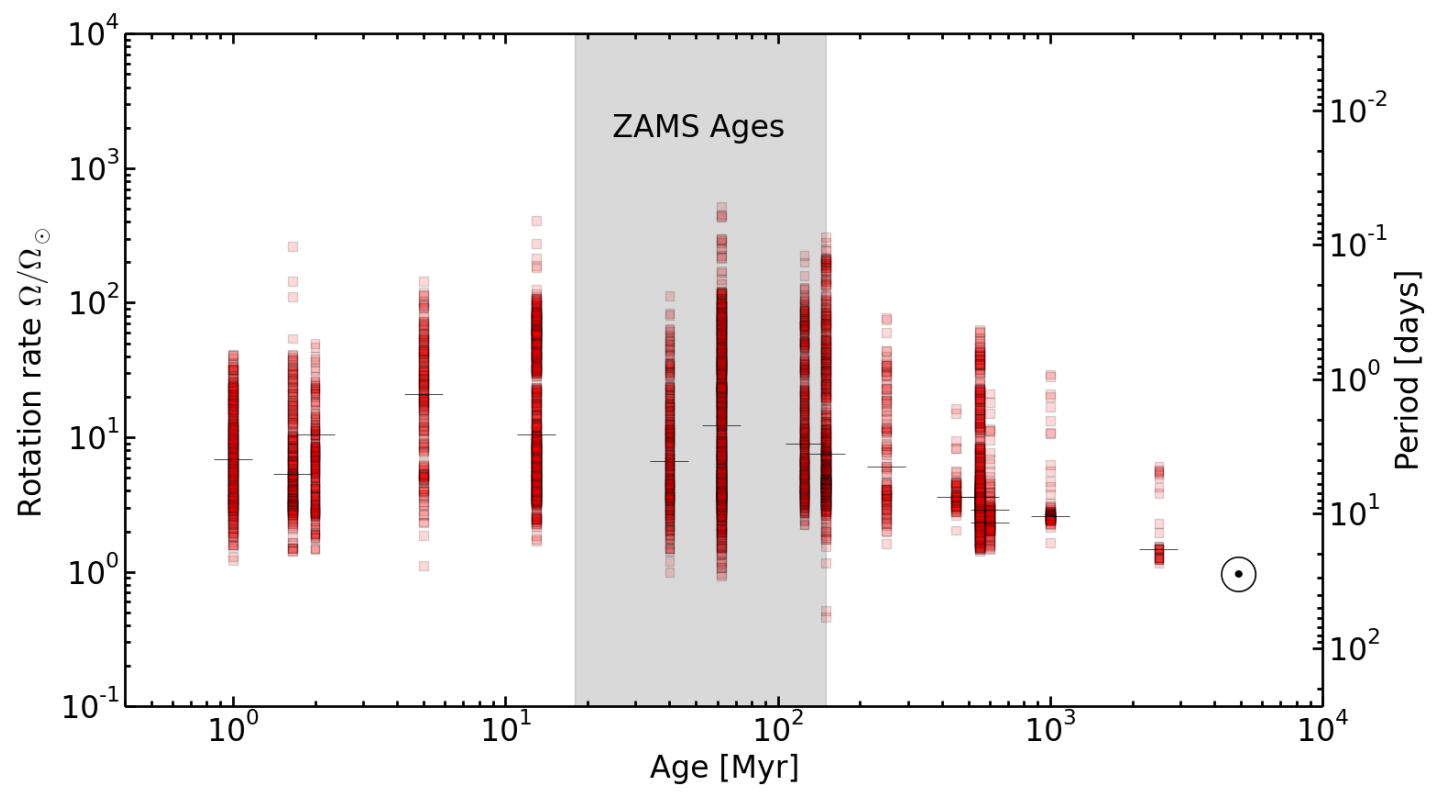

Figure 1.1: A compilation of rotation periods from open clusters of various ages, stretching from the 1 Myr Orion Nebula Cluster to the 2.5 Gyr NGC6811 cluster in the Kepler field. The medians of the distributions are shown in black, and the zero-age-main sequence ages for the mass range $0.5-1.5 \mathrm{M}_{\odot}$ are indicated by the shaded region. A list of clusters and their ages is shown in Table A.1.

1.1 we see that there is indeed a trend of increasing rotation rates from the early PMS toward the region where the majority of stars hit the ZAMS. However, simple conservation of AM would lead to stars rotating with $\lesssim 1$ day periods at the ZAMS (Bouvier et al. 2014), but as shown by Fig. 1.1 the average rotation rate is much slower than this. To achieve such a wide distribution of rotation periods the spin-up rate of the star must be reduced by some additional mechanisms of AM transport. The timescales at which these mechanisms are important and their exact details are poorly understood (see review by Bouvier et al. 2014), but they can be broadly attributed to three different phenomena:

Disk interaction: AM transport away from the star is thought to be able to take place via the so-called 'disk-locking' mechanism (Camenzind 1990, Koenigl 1991, Long et al. 2005). In such a scenario the star's magnetic field is coupled to that of its surrounding proto-planetary disk, whereby AM is transferred outward into the disk. An uncoupled disk would tend toward Keplerian motion which is slower than the stellar surface, and so establishing a coupling leads to the inner disk material being spun-up. Conversely this leads to a reduction of the spin-up already being experienced by the star from simple contraction. This picture has been supported by an observed correlation between the presence of accretion disk indicators (e.g., near infrared excess, Herbst et al. 2007) in the slowly rotating population, while the fast rotators show less evidence for circumstellar material. 
Stellar winds: Stars like the Sun exhibit a stellar wind which consists of charged particles that are thermally and centrifugally liberated from their coronae (Parker 1958). These particles are initially locked to the magnetic field lines out to a radius where the field strength becomes less than the kinetic gas pressure (Weber and Davis 1967, Mestel 1968). At this point (the Alfvén radius) the stellar wind material is released, taking with it AM from the stellar surface and corona. Stars on the PMS are also magnetically active, and so are expected to have stellar winds.

The torque by a stellar wind must be proportional to the rotation rate of the star and the mass-loss rate through a sphere of radius equivalent to the Alfvén radius. Many models currently exist that attempt to put this torque in terms of the fundamental stellar properties: mass $M$, radius $R$ and rotation rate $\Omega$ of the star (Kawaler 1988, Reiners et al. 2012, Matt et al. 2012), so that they may be related to observations of rotation at various stages of stellar evolution. This is complicated however, by the fact that the magnetic field strength and configurations that determine the Alfvén radius are poorly understood. Furthermore, mass-loss rate estimates are very variable, spanning several orders of magnitude for stars of similar spectral type (Wood 2004), making a comparison to model predictions difficult.

Internal redistribution of angular momentum: Little is known about the internal rotation profile of stars on the Hayashi track. Barnes et al. (2005) showed that cool, fully convective MS stars have very weak surface differential rotation. Stars on the Hayashi track have similar characteristics, and so may also show the same behavior. In addition, the convective motions inside the star are able to transport AM radially throughout the star. An angular momentum drain at the surface from, e.g., a stellar wind, which would otherwise lead to differential rotation, would be felt by the stellar interior on the relatively short convective time scales.

Once the star reaches the base of the Hayashi track it will start to develop a radiative interior, at which point convection can no longer transport AM in the stellar core. This prompts the so-called double-zone model, where the radiative core and convective envelope rotate as solid bodies, but at different rates. This is a rough approximation to the solar rotation profile, where the convective envelope on average rotates slightly slower than the core (discussed further in 1.1.3).

Complete decoupling would mean that an AM drain at the surface only has to spin down the envelope, while complete coupling (solid-body rotation) would mean the AM drain has to spin down the entire star. Varying degrees of coupling strength between the two zones could then potentially explain some of the scatter in the rotation rates seen near the ZAMS. However it is unclear what mechanism facilitates such an exchange of AM. Potential explanations range from magnetic interaction (Maeder and Meynet 2004), to transport through internal gravity waves (see, e.g., Marques et al. 2013).

\subsubsection{Main-sequence rotation}

Once a solar-mass star arrives on the MS it will have initiated hydrogen burning in the core, and will also have established a radiative interior with a thick convective envelope. The gravitational collapse will have ceased and the structure of the star will not change remarkably for the remainder of the MS lifetime (Kippenhahn et al. 2012). This means that the spin-up seen on the PMS will stop. However, the magnetically driven stellar wind 


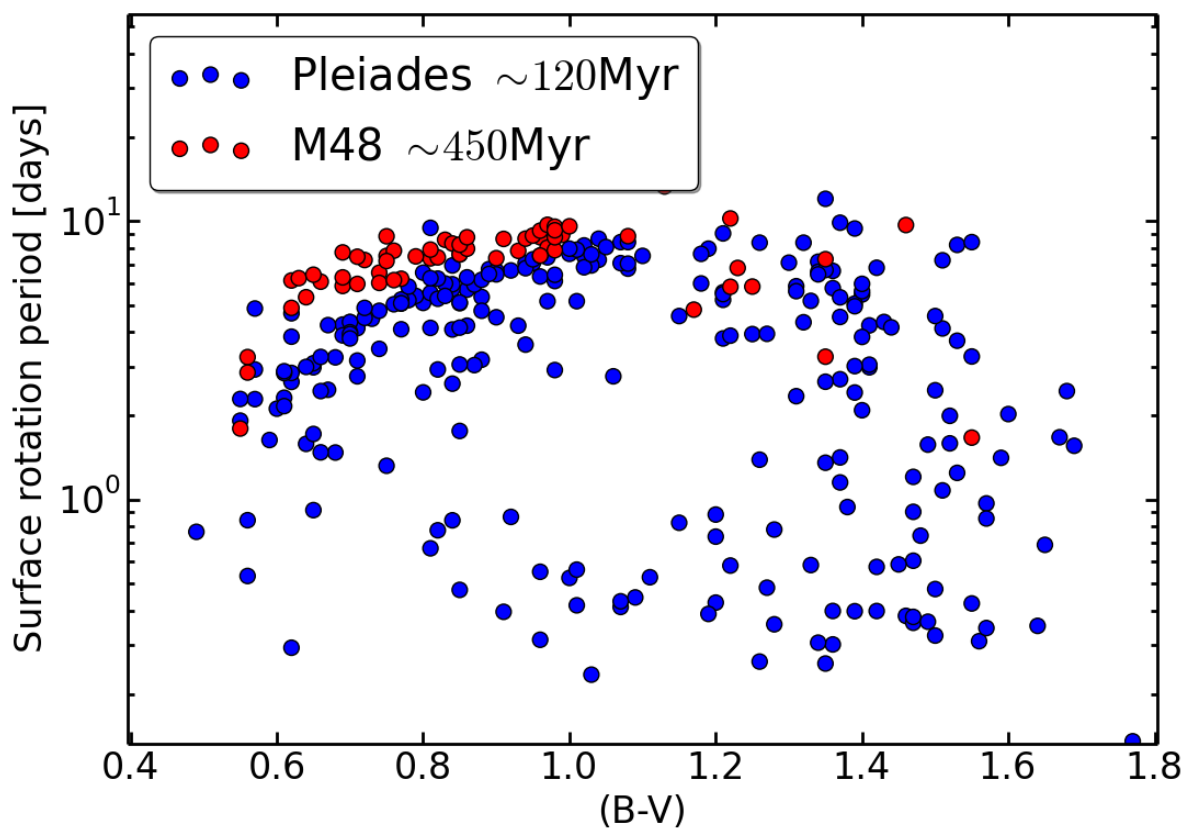

Figure 1.2: Rotation periods as a function of (B-V) color index for the Pleiades (Hartman et al. 2010) and M48 (Barnes et al. 2015) clusters.

which has been present during the majority of the PMS lifetime will keep draining AM from the star, causing the surface rotation rate to slowly decrease. At the ZAMS the stars are broadly distributed around periods $\lesssim 15$ days, but Fig. 1.1 shows that the rotation rates begin to converge onto a single decay law only a few hundred Myr after this.

This convergence toward a main rotation sequence can also be seen in Fig. 1.2, which shows the rotation periods as a function of $(\mathrm{B}-\mathrm{V})$ color index ${ }^{1}$ of the Pleiades and M48 clusters. Here, the younger Pleiades cluster has a large scatter in rotation periods, but with a sequence of stars grouped at long periods. The M48 cluster is much older and predominantly consists of stars that have converged onto the main rotation sequence. These same features are seen in other clusters of various ages (Barnes 2003). The cluster members move toward a single well-defined main rotation sequence as the cluster ages. The more massive stars appear to reach the main rotation sequence before their less massive counterparts. Barnes (2003, and subsequent publications) suggested the existence of two distinct timescales that are relevant for the stellar spin-down of stars near and past the ZAMS. The initial spin-down takes place over a few tens of Myr, increasing with a decrease in the mass of the star. Once the star reaches the main rotation sequence the relevant timescale for the spin-down increases to several Gyr. This could imply two different mechanisms that dominate the early spin-down of a star (Barnes 2010), although the exact nature of such mechanisms are unknown.

Another clear feature in Fig. 1.2 is that the average rotation periods generally decrease when moving toward lower $(B-V)$ values, corresponding to hotter, more massive stars.

\footnotetext{
${ }^{1} \mathrm{~A}$ proxy for mass, increasing toward lower $(\mathrm{B}-\mathrm{V})$ values
} 
For $(B-V) \lesssim 0.5$ the rotation periods decrease dramatically ${ }^{2}$. Past this narrow range in mass, known as the 'Kraft break' (Kraft 1970) the stars appear to lose their ability to spin down. This coincides with a thinning of the surface convection zone, which eventually disappears completely. The presence of a convective envelope has been thought to be one of the main drivers for generating strong magnetic fields (see, e.g., Schatzman 1962), and thus a magnetically driven stellar wind.

\subsubsection{Solar rotation}

Our knowledge of the solar rotation profile is the most detailed picture of rotation that we have for any star. Some of the first observations of solar rotation with sunspots include those by Johannes Fabricius, Galileo Galilei, and, Christopher Scheiner in the 17th century. Since then the study of solar rotation has advanced considerably, using photometric imaging, spectroscopy and spectropolarimetry.

The methods used to study the rotation of the Sun can be broadly divided into three groups, which are also in some variation applicable to stars. These are: spectroscopic radial velocity measurements, tracing of surface features across the solar disk, and helioseismology. The two former methods are predominantly sensitive to the surface, while helioseismology probes rotation in the deep solar interior. Notable observatories dedicated to helioseismology include the space based missions SoHO (Domingo et al. 1995), SDO (Pesnell et al. 2012), and the ground based observation networks GONG (Harvey et al. 1988) and BiSON (Chaplin et al. 1996).

Surface rotation: The first observations of surface differential rotation on the Sun were performed by measuring the rotation of sunspots as they cross the solar surface. The rotation rate of spots near the equator was seen as being faster than those at higher latitudes. Today, the variation of the solar rotation with latitude is typically represented by $\Omega(\theta) / 2 \pi=A+B \sin ^{2} \theta+C \sin ^{4} \theta$ (Howard and Harvey 1970), where $\theta$ is the solar latitude, $A$ is the equatorial rotation rate and $B$ and $C$ define the rate of decrease with latitude. There have been multiple studies of rotation (reviewed by Beck 2000). Interestingly, each method returns different values of the $\Omega(\theta)$ coefficients, e.g., the equatorial rotation rate $A=453.75 \mathrm{nHz}$ (25.51 days) as measured by spectroscopy and $A=473.01 \mathrm{nHz}(24.47$ days) as measured by surface tracers (Snodgrass and Ulrich 1990). This might reflect the sensitivity to rotation of each method varying with depth in the Sun. The spectroscopic methods are primarily sensitivity to depths at which the surface plasma becomes optically thick at a given wavelength (the photosphere), while the surface tracers may be rooted deeper inside the Sun, and so feel the rotation at this depth. The surface tracer rotation rates appear to match those observed via helioseismology at depths immediately below the photosphere (Beck 2000, see Fig. 8), but may well be a weighted average of a broader range of depths.

Differential rotation in the convection zone: Helioseismology is the only tool available for probing the rotation of the solar interior. Briefly, helioseismology studies the acoustic oscillations of modes that propagate through the solar interior. These waves are

\footnotetext{
${ }^{2}$ Note that the lack of points in this region of Fig. 1.2 is a selection bias in the chosen catalogs.
} 


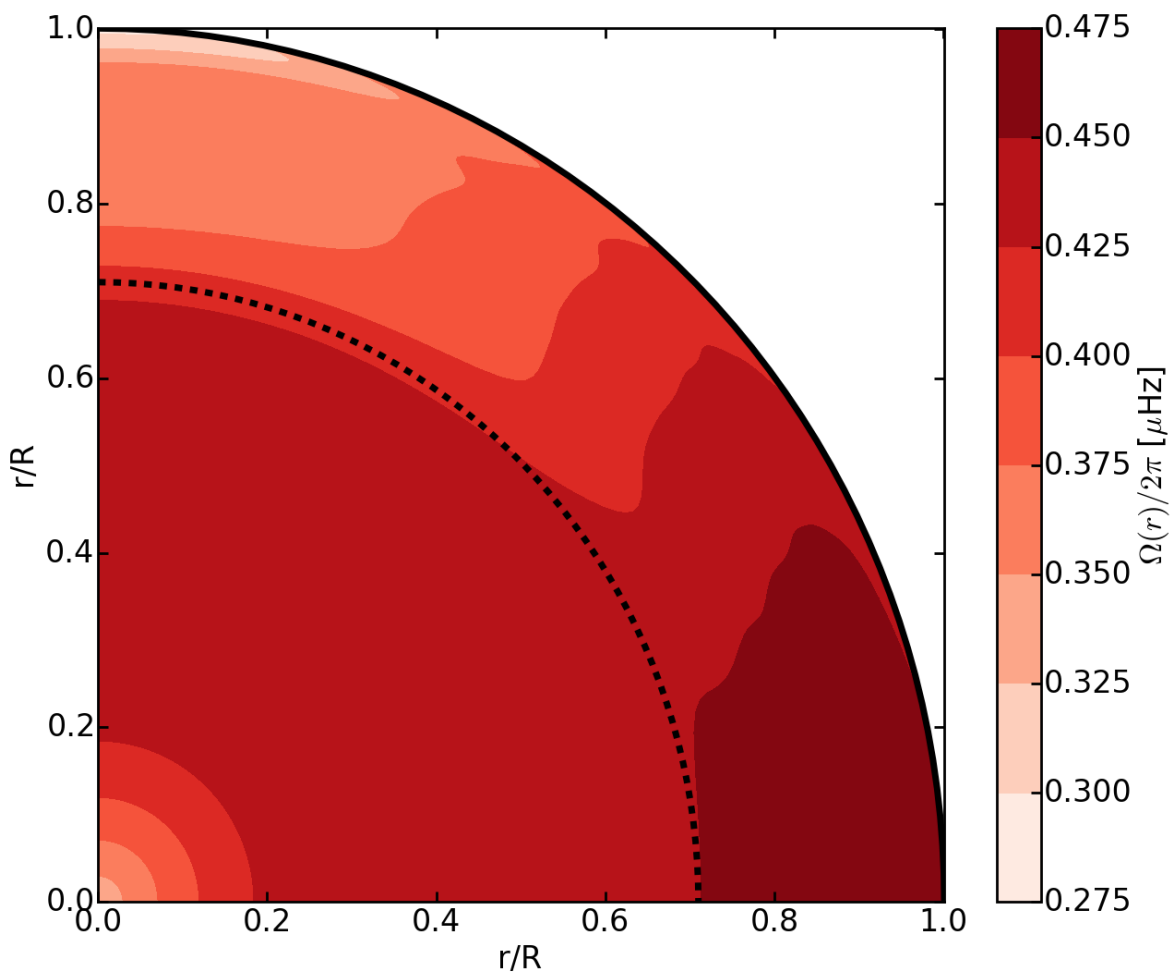

Figure 1.3: The 2D solar rotation profile derived from helioseismic inversions (data adapted from Larson and Schou (2015)). The profile is symmetric around the equator and does not vary with longitude. The measurements are insensitive to the high polar latitudes $\left(>75^{\circ}\right)$ and the deep core (below $r \approx 0.3 R_{\odot}$ ), the values shown here should therefore not be taken as representative of the true rotation rates in these regions. The dashed line denotes the base of the convection zone.

perturbed by rotation, and so reveal the internal rotation rates at various radii and latitudes. The principles and concepts of this method are largely identical to those of asteroseismology $^{3}$, which will be discussed in further detail in Sect. 1.2.4.

Figure 1.3 shows the 2D solar rotation profile (Schou et al. 1998), which is the result of helioseismic inversion of the perturbations to the acoustic oscillations. The convection zone, located between the surface and the dashed curve, shows a strong latitudinal variation similar to that seen by surface measurements. A relatively thin shear layer exists near the surface which spans only a few percent of the outer solar radius. In this layer the rotation rate increases inward for latitudes below $\sim 70^{\circ}$, and above this latitude the shear initially becomes very weak and then changes sign at higher latitudes (see, e.g., Barekat et al. 2014).

Below the near-surface shear layer the rotation rate becomes approximately constant with radius, having only a small radial gradient. The latitudinal gradient similar to that of the surface is maintained throughout a large part of the convection zone. Just below the convection zone lies a stronger shear layer. This layer, called the tachocline, is believed

\footnotetext{
${ }^{3}$ Traditionally helioseismology only refers to seismology on the Sun, whereas asteroseismology refers to seismology of other stars.
} 
to be one of the main drivers of the solar magnetic dynamo (see Sect. 1.3.3). Basu and Antia (2003) find that the tachocline is located at a radius of $0.69 \mathrm{R}_{\odot}$ and has a width of $0.02 \mathrm{R}_{\odot}$, but speculate that it might move slightly outward in radius and become somewhat narrower at higher latitudes. It should be noted that the inversion technique used to produce Fig. 1.3 introduces a degree of smoothing to sharp features (Beck 2000), so the transition to solid body rotation may be sharper than it appears. As is seen in Fig. 1.3 the scale of the tachocline shear varies with latitude. Below $\sim 35^{\circ}$ latitude the rotation of the envelope is marginally faster than that of the interior, whereas for higher latitudes the rotation gradient in the shear layer changes sign, producing a slower rotating pole. However, the average rotation rate in the convective envelope is about $50 \mathrm{nHz}$ slower than in the radiative interior.

Rotation in the deep interior: Beneath the tachocline the rotation profile transitions from the differentially rotating envelope, into the solidly rotating radiative interior. The helioseismic measurements indicate no gradients in the rotation profile down to $\sim 0.3 \mathrm{R}_{\odot}$, at which point the measurement uncertainties increase dramatically. At this point the acoustic oscillations used to measure rotation in the convection zone have lost much of their sensitivity to rotation, hence the large uncertainties in the rotation rate. However, the solid-body rotation profile is thought to continue down to the solar core (Howe 2009).

Driving differential rotation: Much work is currently being done to understand the exact mechanisms for establishing and maintaining the differential rotation profile seen in the Sun (see the review by Miesch 2005, and references therein). The angular momentum is assumed to be redistributed in four ways: meridional circulation which is a circulatory flow in the radial and latitudinal direction, convective motion in the radial direction, magnetic forces, and viscous diffusion (see Eq. 9-16 in Thompson et al. 2003). The location and role of each of these terms however is still uncertain. While the magnetic and viscous diffusion terms are likely small in the convection zone, not much is known about the conditions in or near the tachocline where the magnetic term may become more important.

\subsubsection{Differential rotation in other stars}

Naturally, since the Sun rotates differentially one must expect that other stars can do the same. In the past the majority of work done on stellar rotation has focused simply on measuring the mean surface rotation rate, and furthermore assuming solid-body rotation. However, recently a few studies have managed to find evidence for differential rotation. The methods involved typically have very stringent measurement criteria, and so no single star has a complete description of both its radial and latitudinal rotation profile. In the following these two components are therefore reviewed separately.

\subsubsection{Latitudinal differential rotation}

The majority of work done on stellar differential rotation has focused on the surface rotation rates. Early work by Donati and Collier Cameron (1997) imaged surface features crossing the stellar disk using variations in spectral lines (called Doppler imaging). The 
presence of spots on the stellar surface creates perturbations to the spectroscopic line profiles depending on the latitude and longitude of the spot. Doppler imaging can be used to gauge the rotation rate at various latitudes, and subsequently estimate the amount of differential rotation (see the studies reviewed by Barnes et al. 2005). However, Collier Cameron (2007) notes that the errors associated with this method are often difficult to quantify, and in some cases are underestimated.

Another method for estimating the surface differential rotation is through direct modeling of the effect of rotation of spots across the stellar disk on the integrated light curves (see, e.g., Fröhlich et al. 2009, Walkowicz and Basri 2013, and also Sect. 1.2.3.1). However, this method assumes that all variability is caused by well-defined surfaces spots, and typically relies on several degenerate parameters such as the spot area and temperature contrast with the photosphere, or the latitude of the spot and the stellar inclination angle.

Reiners and Schmitt (2002) developed a Fourier-transform based method for estimating the latitudinal shear solely by the spectroscopic line shape. They calculated the deviation of the line profiles from that of a rigidly rotating surface, and use this to estimate the scale of the radial shear. The benefit of this method is that it does not require long observing campaigns or that the star shows any spot variability. However, this method is only applicable to fast rotators $(\gtrsim 10 \mathrm{~km} / \mathrm{s})$ where the lines are broadened enough to be well resolved.

Using the above mentioned Fourier method and collating the results of Barnes et al. (2005), Reiners (2006) confirmed that the surface latitudinal shear increases with increasing temperature, as predicted by stellar mean field modelling (Küker and Rüdiger 2005). However, they also note that even this combined sample is biased toward the young, rapidly rotating, magnetically active cool stars, and the hot rapidly rotating F-stars; specifically lacking stars of similar age, rotation rate and spectral type as the Sun.

\subsubsection{Radial differential rotation}

The internal rotation profile of stars can only be probed by seismology. This was first done on massive B-type stars by Aerts et al. (2003), Pamyatnykh et al. (2004) and Briquet et al. (2007). More recently, using data from the NASA Kepler mission (Borucki et al. 2010), Beck et al. (2012), Mosser et al. (2012) and Deheuvels et al. (2012) were able to measure the core rotation of several red giant stars, at various stages along the red giant branch. At the end of the MS lifetime of a Sun-like star, the convection zone expands while the radiative core contracts. In principle this would lead to a rapidly rotating core and a slowly rotating envelope. These studies showed however, that while the core rotation rate of red giants is still much faster than the envelope, it is approximately an order of magnitude slower than what would be anticipated by current models for rotation evolution on the post-main-sequence (Eggenberger et al. 2012, Cantiello et al. 2014).

The picture of internal rotation has lately been further complicated by other studies. Kurtz et al. (2014) found a pulsating F-type star which showed evidence of an envelope rotating faster than interior, in contrast to what we see in the Sun. Moreover, Triana et al. (2015) measured the core rotation of a young B-type star where a counter-rotating core and envelope configuration appear the most likely scenario.

The number of stars that have been studied with asteroseismology currently number in the thousands, but only a handful have measurements of internal rotation. Furthermore, 
these are all more evolved or more massive than the Sun (see the review by Aerts 2015), which makes constraints of stellar rotation models along the MS very difficult.

\subsection{Measuring stellar rotation with Kepler}

There are several ways to measure the rotation of a star. This section is an introduction to the data and methods used in the following chapters. These are: the use of photometric variability caused by surface features, and measuring the effects of rotation on the oscillation frequencies with asteroseismology.

\subsubsection{Kepler photometry}

The Kepler mission was designed to measure the occurrence rate of earth like planets around Sun-like stars ${ }^{4}$ (Borucki et al. 1997). This requires observation of the same star field $^{5}$ over several years, since an Earth-like planet would by definition have a 1 year orbital period. The nominal mission spanned approximately 4 years from 2009 to 2013 , at which point two of the on-board reaction wheels had failed, rendering the spacecraft unable to maintain high precision pointing on the Kepler field.

The observations from the Kepler satellite are photometric measurements in a wavelength band from approximately 4000-9000A. The observing campaign was divided into 3 month segments (quarters), with a short break for spacecraft reorientation in between. During each quarter a star was assigned a pixel mask, where the flux values of each pixel are stored at each cadence. The exposure and readout time for the CCDs is 6.54 seconds. The spacecraft observed in two modes: a short cadence mode (SC) where the exposures were binned up to $\sim 58$ seconds, and a long cadence (LC) mode binned to 29.45 minutes. Approximately 150, 000 stars were observed in LC mode during each quarter, while only up to 512 stars were observed in SC mode because of data constraints. Each quarter the target list was modified according to scientific requirements, eventually leading to approximately 200, 000 stars being observed.

Kepler targets are labeled with KIC (Kepler Input Catalog), followed by a nine digit number string. In principle this number string refers exclusively to a single star, however, in some cases close binary systems or background stars may also be captured in the photometric aperture (Appourchaux et al. 2015).

The main data product of the mission consists of simple aperture photometry time series, where the apertures within the pixel masks are automatically computed to optimize chances of detecting exoplanet transits. An example of a light curve from KIC006106415 is shown in Fig. 1.4. The data from Kepler is publicly available ${ }^{6}$ in two formats: the uncorrected simple aperture photometry, and the automatically corrected PDC_MAP/msMAP photometry (see Stumpe et al. 2012, Smith et al. 2012, for details and discussion on the correction pipeline, as well as Appendix B). In the following work we predominantly used the corrected photometry, since many now well-known systematic effects are removed. In chapter 2 we used the LC data for all the available stars in the Kepler catalog, while in

\footnotetext{
${ }^{4}$ Sometimes denoted the $\eta$-Earth parameter

${ }^{5}$ Centered on $\alpha=19 h 22 m 40 s \delta=+44^{\circ} 30^{\prime} 00^{\prime \prime}$, covering part of the Cygnus constellation.

${ }^{6}$ https://archive.stsci.edu/kepler/
} 


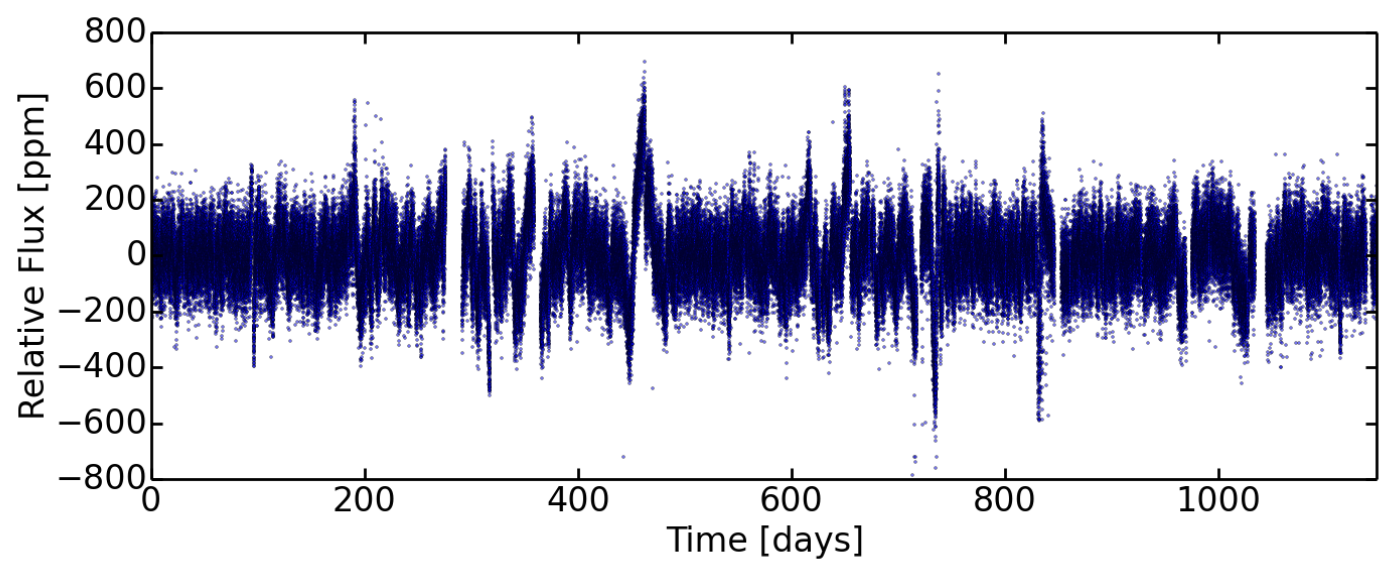

Figure 1.4: Short cadence light curve from KIC006116048 over the duration of the nominal Kepler mission.

chapters 3 and 4 we used both the LC and SC data for six hand-picked stars. See table 4.1 for the characteristic parameters of these stars.

\subsubsection{Photometric time series analysis}

In the following work the methods for measuring rotation are based on asteroseismology and periodic variability in stellar light curves. Both of these are in turn based on treating the frequency content of the time series by computing its power spectrum. It is therefore appropriate to first discuss the general properties of time series analysis and the way in which a power spectrum is constructed.

The minimum observation time $T$ required to identify a periodicity is its period $P$; or conversely one can only completely determine periods when $P \leq T$. Similarly the Nyquist criterion specifies the shortest period that can be correctly identified given a certain sampling. Attempting to reconstruct a sine wave from a set of samples at intervals $\Delta t \ll P$ will result in many samples per period. Reducing the sampling to $2 \Delta t=P$, however, provides only two samples per period, at which point it no longer becomes possible to define the original sine wave by the sampling points, i.e., one would be sampling at the same phase of each successive oscillation, making the measurements indistinguishable from a series of constant values.

In frequency this corresponds to having a range of physically meaningful frequencies from $v_{0}=0$ to $v_{\text {Nyquist }}=1 /(2 \Delta t)$, with a resolution of $1 / T$.

\subsubsection{The Lomb-Scargle periodogram}

The primary tool for time series analysis used in the following work is the Lomb-Scargle (LS) method for spectral analysis (Lomb 1976, Scargle 1982). The LS method functions much like a discrete Fourier transform in that it allows one to construct a spectrum of the frequency content of a time series. The variant of the LS method used here is that of Frandsen et al. (1995) which is based on $\chi^{2}$ minimization, where for a time series consisting of data $D$ at times $t$ the model consists of a sine wave $m=A \sin 2 \pi v t+\delta$. The 


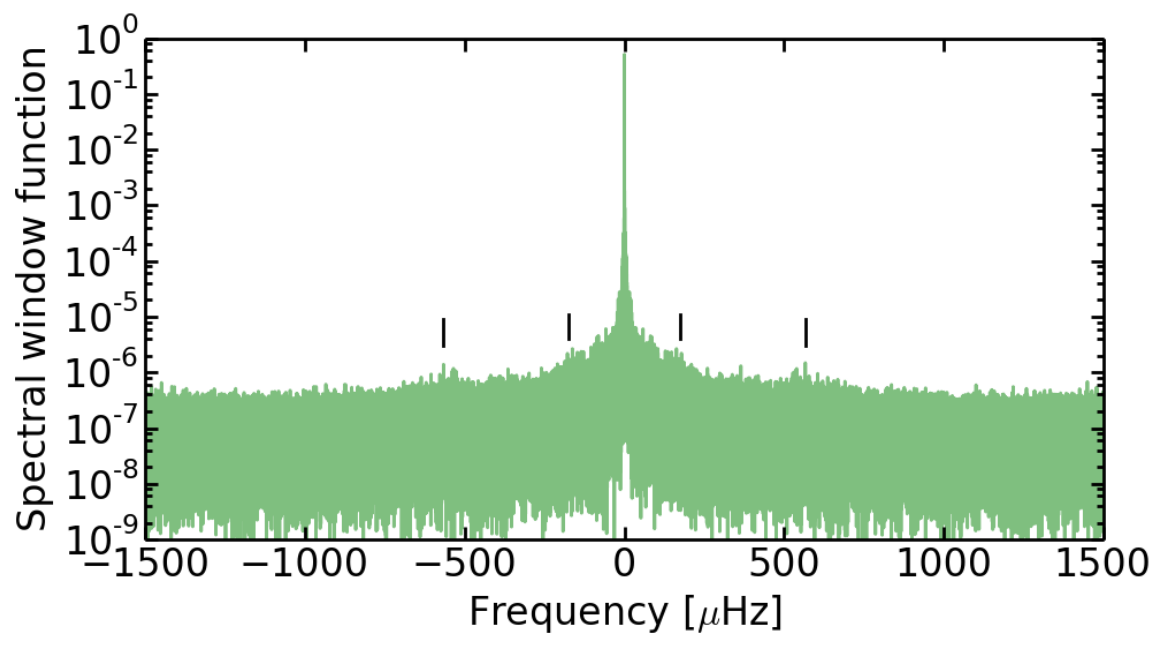

Figure 1.5: Spectral window function of the time series from KIC006116048. For a perfect window function this should be a function proportional to $\sin ^{2}(v) / v^{2}$ (or $\sin ^{2} v$ ). The most pronounced deviations from a $\operatorname{sinc}^{2}$ function are the side-lobes at $\pm 174 \mu \mathrm{Hz}$ and $\pm 566 \mu \mathrm{Hz}$ indicated in black. The former is an artifact of the reaction wheel heating cycle, and the latter is the frequency of the long cadence sampling. This is likely caused by the on-board electronics (Christiansen et al. 2013). Because of their scale relative to the central peak they are deemed inconsequential.

details of the derivations and calculation of the power spectrum are shown in Kjeldsen (1992).

In the case that $D$ is a regularly sampled time series (i.e., $\Delta t$ is fixed) the above power spectrum becomes equivalent to that computed by using the discrete Fourier transform, or the more commonly used fast Fourier transform (FFT). The FFT is generally preferable because of its speed and easy implementation, but requires equidistant sampling of the time series. In real-world scenarios where observations may be interrupted or delayed because of, say, clouds, technical failures, or sleepy astronomers, the sampling rate will naturally vary. The Kepler data used here are almost equidistant, but a small change in the cadence times is apparent over the course of the mission lifetime, making the LS method the preferred means of computing the power spectrum.

Gaps in the time series also impact the shape of the power spectrum. The effect of randomly missing cadences is to simply decrease the signal-to-noise ratio $(S / N)$. Periodic gaps however are more troublesome in that they produce alias peaks surrounding the true periodicity. These peaks are separated from the frequency of the real variability by the frequency of the gaps. This has important implications for multi-night ground-based observing campaigns, since they will not be able to observe periods of $\sim 1$ day periods $(11.57 \mu \mathrm{Hz})$, or easily distinguish multiple periods with this separation. The effect of gaps can be estimated by the spectral window function, which corresponds to the spectrum of a sine wave given the sampling rate of the time series. The window function for the SC time series of KIC006106415 is shown in Fig 1.5.

One last notable complication when studying non-sinusoidal variability like starspots is the presence of harmonics in the power spectrum. The LS method assumes the signal 

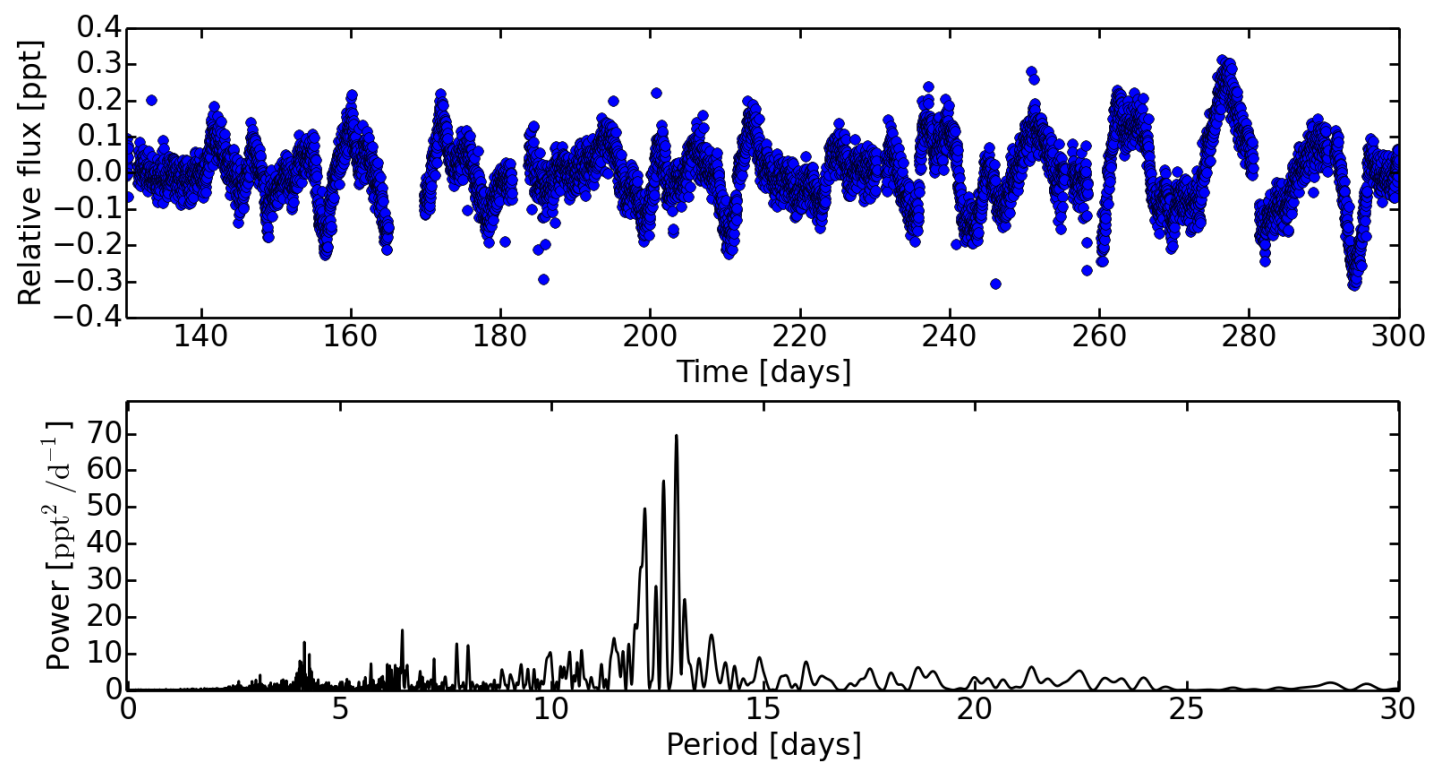

Figure 1.6: Top: Section of a 1450 day time series from KIC010963065, showing the variability from surface features as the long period oscillation. Bottom: Lomb-Scargle periodogram of the time series, showing the rotation period as the primary peak at $\sim 12$ days and a harmonic at $\sim 6$ days.

shape is that of a sinusoid, and so it cannot perfectly fit non-sinusoidal variability. Nonsinusoidal signals will therefore show multiple peaks in the power spectrum at harmonic frequencies of the true rotation period. In the Kepler data this is an issue when dealing with the corrected time series, since long periods tend to be strongly reduced by the PDC pipeline. Harmonic peaks (at shorter periods) may then appear to be the dominant period.

\subsubsection{Measuring rotation from surface variability}

Tracing the movement of spots and other surface features remains one of the simplest and also oldest methods for studying stellar rotation. The idea is simply that as a star rotates, any features fixed to the stellar surface will rotate along with the star, thus periodically dimming or brightening the star slightly as it passes in and out of view. If the period of this variability can be measured the stellar rotation rate is then known.

On the Sun the most prominent visible features that may be used to measure rotation are sunspots. These consist of localized regions of strong magnetic fields in the solar photosphere. The magnetic pressure suppresses convective motions and thus the total outward energy transport, making that local region appear cooler and darker compared to the rest of the photosphere (e.g. Rempel and Schlichenmaier 2011).

Other stars however, only appear as point sources, and variability can in principle not automatically be attributed to active regions. Examples of variability that may appear similar to spot variability are: long period pulsations in hot stars like $\beta$ Cepheid variables and slowly pulsating B stars (see e.g. Aerts et al. 2010, Chapter 2.), or local changes of surface opacities in hot stars (Wraight et al. 2012). These types of variability are however only prevalent in hot stars that do not have surface convection zones. Cool, MS stars 
do not have pulsations on timescales similar to the rotation periods, and are not usually expected to show variability than can appear similar to that from active regions.

Figure 1.6 shows a section of the light curve (top) from KIC010963065, where the variability from the surface features is very clear, but the mean period is not particularly evident because of the lifetime of the variability. Surface features appear randomly in latitude and time, first growing and then decaying with some characteristic timescale. To measure a reliable rotation period from surface variability the lifetime of what causes the variability must be on the order of a few rotation periods or more. On the Sun the spot lifetimes are usually less than a single rotation period, and the rotation period is therefore not evident from the integrated light curves. The power spectrum of KIC010963065 in the lower panel of Fig. 1.6 clearly shows the mean rotation period, where the width of the peak is in part caused by evolution of the signal.

\subsubsection{Measuring differential rotation with surface variability}

Measuring the surface differential rotation of a star from integrated light is straightforward provided the surface features are very persistent. If two or more surface features appear on the star, either simultaneously or at different times, one can in principle measure two or more rotation periods. The difference between the rotation periods then gives the degree of surface shear between the latitudes at which the spots are located. However, this distance in latitude is usually an unknown quantity, making it difficult to estimate the total shear across surface of the star.

In the power spectrum surface differential rotation would produce several closely spaced peaks, which may appear as a single broadened peak. However, this same effect may appear if the average lifetime of the surface features is short, i.e., a localized signal in the time series produces a wide peak in Fourier space. This is also illustrated in the bottom frame of Fig. 1.6, where the power at the rotation period is spread over an interval of $\sim 1$ day. Obviously this complicates the detection of differential rotation. The hare-and-hound study by Aigrain et al. (2015) compared several different methods of analyzing simulated light-curves designed to mimic Kepler data. They found that none of the investigated methods (adaptations of those by Reinhold and Reiners 2013, McQuillan et al. 2014, García et al. 2014, Lanza et al. 2014) could accurately distinguish the evolution of the surface features from the surface differential rotation. This means that any broadening of the peaks in the power spectrum must be assumed to be some average of the signal lifetime and differential rotation.

\subsubsection{Measuring rotation from asteroseismology}

Asteroseismology is to date the only means we have of peering into stellar interiors. This has been done with great success in the Sun, where it is used to study everything from convective motions and magnetic fields near the surface to ionization regions and rotation in the deep interior. Because of its proximity the solar surface can be spatially resolved, showing millions of oscillation modes, providing an extremely detailed picture of the solar interior. Other stars however are only point sources, and so we only see the diskintegrated light. This dramatically reduces the number of visible modes. 
The oscillation pattern on the surface of a star can be decomposed into a sum of spherical harmonic functions, where each oscillation is defined by an angular degree $l$ and the azimuthal order $m$, as well as an additional number for the radial order $n$. The radial order defines the number of nodal points of the oscillation in the radial direction, while $l$ and $m$ give the nodal lines in the latitudinal and azimuthal directions.

In a Sun-like star the pulsations are stochastically damped harmonic oscillations, where the driving and damping mechanism is the convective motion in the outer envelope. For these modes the restoring force is the local gas pressure and so they are typically denoted as $p$-modes or acoustic modes. These are distinguished from the modes excited in the deep stellar interior, called $g$-modes, where the restoring force is gravity. The amplitude of the $g$-modes are strongly damped in a convectively unstable medium, and so they are not typically visible in Sun-like stars, i.e., they never reach the surface. There is currently no clear evidence for $g$-mode pulsations in the Sun.

Figure 1.7 shows an oscillation spectrum of KIC006116048. The $p$-modes appear in a regularly spaced pattern of different radial orders. The left inset shows a zoom on a set of modes with $(n, l=2),(n+1, l=0),(n, l=3),(n, l=1)$. For $l>0$ the peaks are multiplets of $2 l+1$ azimuthal orders.

Modes with $|m|>0$ are traveling waves that move around the star in prograde and retrograde directions. When the star rotates the frequencies of these modes become Doppler shifted. The frequencies of these modes are therefore perturbed, or 'split', from that of the $m=0$ mode by an amount proportional to the rotation rate of the star. For the particular case shown in Fig. 1.7 the rotational splitting is only very slight, and so the multiplets only appear as broadened peaks as seen in the right inset. The rotational splitting can be measured by fitting a model to the oscillation spectrum, thereby revealing the rotation of the star.

Modes with degree $l>3$ experience strong cancellation when viewed in integrated light; as one part of the stellar surface moves outward another corresponding part moves inward, giving almost a net zero change in the emitted light. While the few visible modes are still enough to obtain a large amount of information about the stellar structure and evolutionary state, it makes discerning differential rotation difficult.

\subsubsection{Measuring differential rotation with asteroseismology}

As was seen in Fig. 1.3 the rotation rate inside a star may vary with both radius and latitude. The oscillation modes in principle feel all of the interior of the star, but with varying degrees of sensitivity in both radius and latitude. The frequency of a given mode $v_{n l m}$ in the oscillation spectrum can be written as

$$
v_{n l m}=v_{n l}+m \int_{0}^{\pi} \int_{0}^{R} K_{n l m}(\theta, r) \Omega(\theta, r) d r d \theta,
$$

where $v_{n l}$ is the mean frequency of the multiplet ${ }^{7}$, and $\Omega(\theta, r)$ is the rotation profile of the star. $K_{n l m}(\theta, r)$ is the rotation sensitivity kernel which is computed from the stellar structure model by

\footnotetext{
${ }^{7}$ Often assumed to be the frequency of the $m=0$ mode
} 


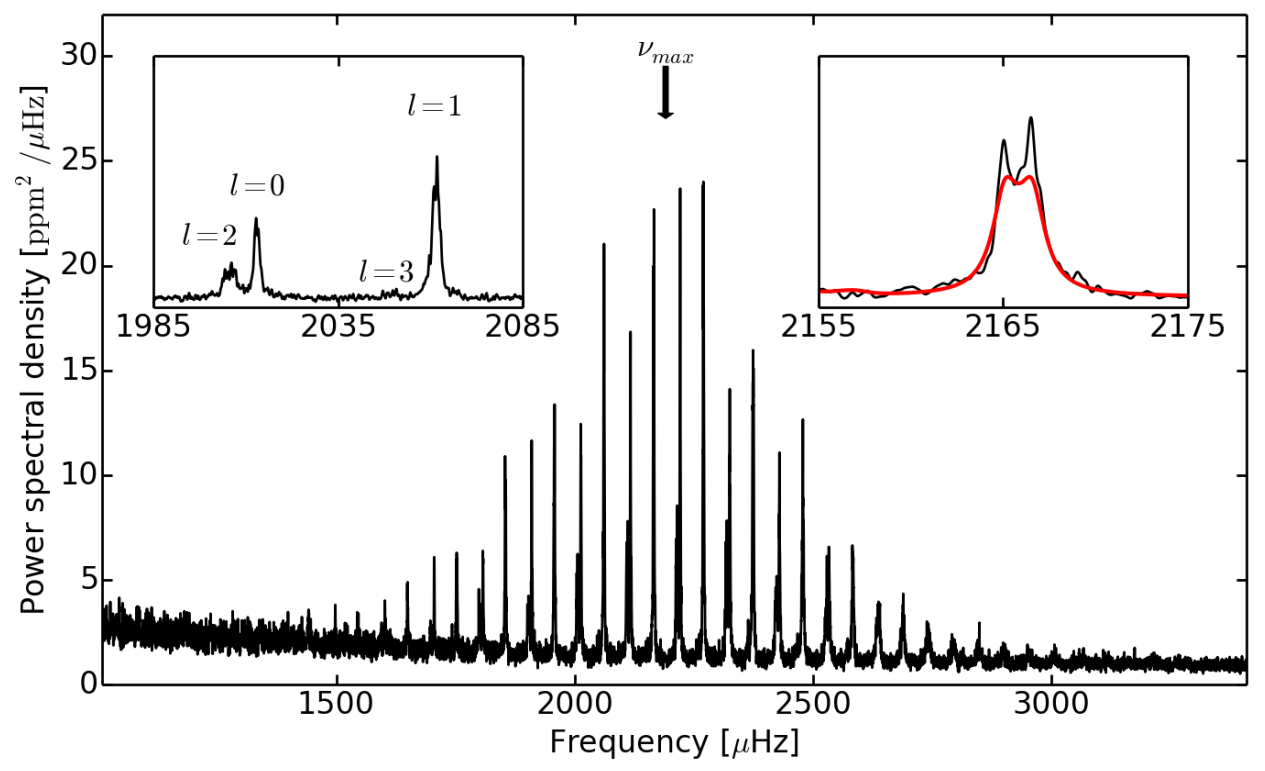

Figure 1.7: Power spectrum of KIC006116048 smoothed with a $0.1 \mu \mathrm{Hz}$ Gaussian kernel, centered at the $p$-mode envelope. The Gaussian shape of the mode heights is evident, with the height of the different angular degrees modulated by the respective visibilities. The frequency of maximum oscillation power is denoted by $v_{\max }$, and is typically estimated by fitting a Gaussian to the envelope. The left inset shows a zoom on a set of $p$-modes, illustrating the relative positions of modes with $(n, l=2),(n+1, l=0),(n, l=3),(n, l=1)$. The right inset shows a zoom on an $l=1$ multiplet where the splitting is more apparent, with a model shown in red for clarity.

$$
K_{n l m}(\theta, r)=I_{n l}^{-1}\left\{\left(\xi_{r}^{2}-2 \xi_{r} \xi_{h}\right) \mathcal{P}^{2}+\xi_{h}^{2}\left[\left(\frac{d \mathcal{P}}{d \theta}\right)^{2}+\frac{2 m}{\sin ^{2} \theta} \mathcal{P}^{2}-2 \mathcal{P} \frac{d \mathcal{P}}{d \theta} \frac{\cos \theta}{\sin \theta}\right]\right\} \rho r^{2} \sin \theta
$$

where $\xi_{r} \equiv \xi_{r}(r)$ and $\xi_{h} \equiv \xi_{h}(r)$ are the radial and horizontal displacements of the oscillations, $\rho \equiv \rho(r)$ is the mass density, and $\mathcal{P} \equiv P_{l}^{m}(\cos \theta)$ is the associated Legendre polynomial of order $m$ and degree $l$. The mode inertia $I_{n l}$ is given by

$$
I_{n l m}=\frac{2}{2 l+1} \frac{(l+|m|) !}{(l-|m|) !} \int_{0}^{R}\left[\xi_{r}^{2}+l(l+1) \xi_{h}^{2}\right] \rho r^{2} d r .
$$

Figure 1.8 shows the rotation sensitivity kernels for the modes of a single radial order that are visible in a solar-like oscillator. Knowing the mode frequencies and computing $K_{n l m}(\theta, r)$ would in principle allow one to invert for the two dimensional rotation profile of the star. However, the relatively low visibility of the $l=3$ modes limits the majority of latitudinal sensitivity to a region of $\sim 40$ degrees in latitude around the equator, and the radial sensitivity drops off very quickly when moving toward the core.

Because of this insensitivity the rotation profiles of a Sun-like star are often simply assumed to be constant $\Omega(\theta, r)=\Omega=$ const. Equation 1.1 then only contains integrals 

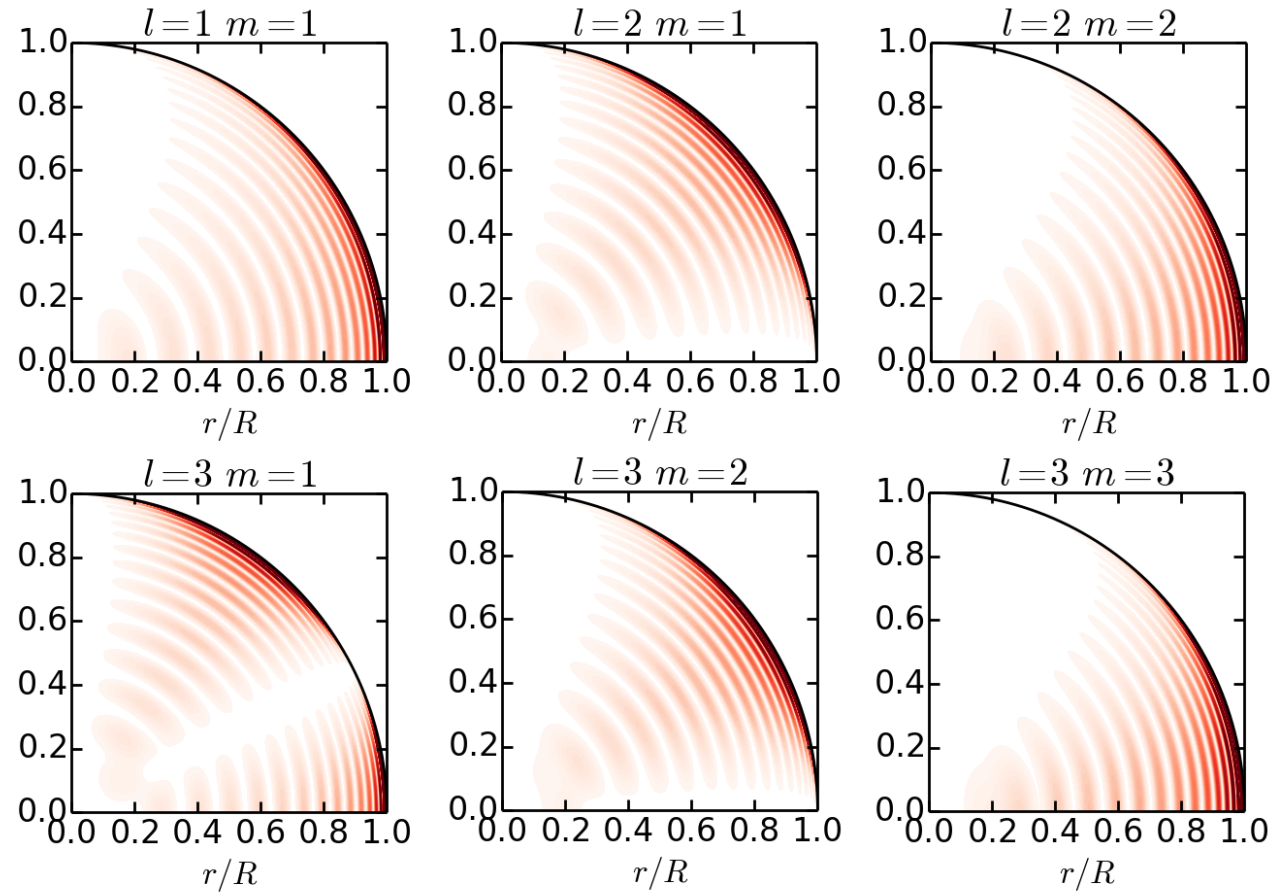

Figure 1.8: Rotation sensitivity kernels $K_{n l m}(\theta, r)$ for the modes of a single radial order that are typically visible in Kepler observations of Sun-like stars. The kernels have no azimuthal variation and are symmetric around the equator (abscissa). The decrease in sensitivity with radius is largely similar for all the different modes. The latitudinal sensitivity differs most noticeably for the $l=3$ modes, but given their relatively low $S / N$ compared to the $l=1$ and $l=2$ modes, the average sensitivity is primarily focused around the equator.

over the $K_{n l m}$. For a Sun-like star where the observed modes are of radial order $n \approx 15$ and above, the integrals over the kernels are approximately equal to unity. Therefore, for a slowly rotating Sun-like star we may make the approximation that

$$
v_{n l m} \approx v_{n l}+m \frac{\Omega}{2 \pi}=v_{n l}+m \delta v,
$$

where $\Omega / 2 \pi$ is equivalent to the rotational splitting which is often denoted by $\delta v$. In most studies this is what is used when fitting oscillation spectra in order to measure the mean rotation of stars (Bazot et al. 2007, Appourchaux et al. 2008, Gizon et al. 2013, Lund et al. 2014a, Davies et al. 2015).

\subsubsection{Peakbagging}

The process of extracting the rotational splitting is often called peakbagging ${ }^{8}$. In essence peakbagging is simply fitting a model to the oscillation power spectrum.

\footnotetext{
${ }^{8}$ The etymology of the term is unknown but likely connected to the mountaineering pursuit of counting the number of climbed or 'bagged' peaks. The term is often falsely credited to Dr. J. Schou who, despite being an avid mountaineer, denies having conceived of the term, but has merely proliferated it.
} 
In the limit of averaging over an infinite number of noise realizations, the peaks in the power spectrum tend toward Lorentzian profiles (Anderson et al. 1990). This is typically known as the limit spectrum. The objective of peakbagging is then to find the set of parameters that best approximate the limit spectrum.

A fit to the spectrum would in principle include three parameters per Lorenztian profile, namely the mode frequency, height, and width, all of which are in principle dependent on $n, l$, and $m$. In Kepler data the typical number of observed radial orders is $\sim 5-10$, with angular degrees potentially up to $l=3$ and for each of those there are $2 l+1$ azimuthal orders. Already, the dimensionality if the parameter space is approaching $\sim 100$, and so without some form of parameterization, attempting to fit the spectrum precisely becomes very difficult and time consuming. The benefit of using a parameterization for a particular set of parameters is that one utilizes information from the entire spectrum to constrain the low $S / N$ parts of the fit. On the other hand this also means that all the relevant parameters become correlated, making interpretation of the errors more difficult.

Mode frequency: The parameterization of the mode frequencies has already been partially covered above. Using a single splitting for all the modes in the spectrum removes the necessity of fitting for the individual $m$-components of a multiplet, drastically reducing number of fitting parameters. This only leaves the central $m=0$ frequencies as free parameters.

In Sun-like stars the separation between modes of the same degree $l$ and consecutive radial orders $n$ varies smoothly with frequency. Stahn (2010) explored using this in order to parameterize the central mode frequencies $(m=0)$ in terms of a low order polynomial. For low $S / N$ this method is very effective for getting robust estimates of the mode frequencies. For high $S / N$ stars like those studied in chapters 3 and 4 however, such a parameterization scheme is unnecessary. Moreover, parameterizing the mode frequencies imposes a 'model' on the spectrum; one that potentially does not allow for higher order variation in the mode frequencies. An example of these are the so-called acoustic glitches which arise from sharp structure changes in the star such as the HII ionization zone and the transition from the convection zone to the radiative interior (see, e.g., Mazumdar et al. 2014). These features often appear as periodic modulations of the mode frequencies with amplitudes on the order of $0.1-1 \mu \mathrm{Hz}$, and so may not be adequately captured by fitting a simple polynomial.

Mode height: The maximum power of a peak in the power spectrum is a combination of many different factors, but can in a broad sense be thought of in terms of the scales involved with the characterizing mode numbers $n, l$ and $m$. Over the frequency range spanned by multiple radial orders, the mode amplitudes $A$ describe the overall power of the peaks and is only dependent on the mode frequency $v_{n l m}$. This is typically seen as a Gaussian variation of the peak power with frequency, with a maximum at a frequency $v_{\max }$ (see Fig 1.7). This Gaussian-like shape is sometimes simply called the $p$-mode envelope. At frequencies much lower than $v_{\text {max }}$ the excitation mechanism is not efficient enough to produce visible modes; while at frequencies higher than $v_{\max }$ the damping of the modes increases strongly. The signal from a strongly damped mode has a short lifetime, corresponding to a broad profile in the power spectrum, leading to lower $S / N$ ratios of these modes. 
Secondly there is the visibility $V_{l}$ of the mode, which decreases with increasing $l$ because of the partial cancellation as described briefly above. The visibility is typically assumed to be a constant value, calculated based on an assumed limb-darkening law and the spectral response function of the observing instrument. The visibility of a particular $l$ is often expressed relative to the visibility of the $l=0$ mode, i.e., $V_{l=1} / V_{l=0} \sim 1.5$, $V_{l=2} / V_{l=0} \sim 0.5$ etc., rapidly decreasing for higher $l$. Given the Gaussian-like shape of the $p$-mode envelope and the ratios of the visibilities, it is possible to parameterize the mode heights in terms of a single Gaussian fit to, e.g., the $l=0$ modes (Stahn 2010). Again this was not deemed necessary for the stars studied in the later chapters given the exceptionally high $S / N$ ratios.

The final effect on the peak power is the geometric modulation $\mathscr{E}_{l m}(i)$, which describes the visibility of the individual $m$-components of a given multiplet due to the viewing angle of the observer, and is given by Gizon and Solanki (2003) as

$$
\mathscr{E}_{l m}(i)=\frac{(l-m) !}{(l+m) !}\left[P_{l}^{|m|}(\cos i)\right]^{2} .
$$

Importantly this gives us the ability to measure the angle of inclination $i$ of the stellar rotation axis, a parameter which, prior to the wide-spread application of asteroseismology, would at best be poorly constrained and often completely unknown.

All in all the peak power may be written as

$$
P_{n l m}=\frac{2 A_{n l m}^{2} V_{l}}{\pi \Gamma_{n l m}} \mathscr{E}_{l m}(i)
$$

where $\Gamma$ is the full width at half maximum of the Lorentzian profile (see below). In the following work the visibility and mode amplitude are not parameters of interest, and together with $\Gamma$ are therefore lumped into a single parameter $S_{n l m}$, so that Eq. 1.6 becomes $P_{n l m}=S_{n l m} \mathscr{E}_{l m}(i)$.

Mode width: The mode width $\Gamma$ is the final parameter needed to describe the Lorentzian. In general the width of a peak in the power spectrum is determined by the duration of the signal in the time series. This can be limited by either the length of the time series or the lifetime of the physical process producing the signal. The Kepler data spans approximately four years, while the typical mode lifetime in a Sun-like star is on the order of a few days. The width of the peaks in the power spectrum is therefore dominated by the mode lifetime.

The mode lifetime decreases with increasing frequency with a shallow local minimum near $v_{\text {max }}$. This variation is approximately smooth and can therefore by parameterized in terms of a low order polynomial. Figure 1.9 shows the line widths (black) of the solar $l=0$ modes as a function of frequency. A low order polynomial (red) is fit to the line widths. The choice of polynomial order is not motivated by any physical quantity, but rather chosen such that it represents the mode widths well. The polynomial is centered around $v_{\text {max }}$ in order to minimize correlation between the polynomial coefficients and thus the mode widths themselves. For a slow rotator the rotational splittings are often on the order of or smaller than the line widths at frequencies higher than $v_{\max }$. This makes it difficult to distinguish the two if only a single mode is analyzed. In the following chapters we therefore opt to use a polynomial parameterization to represent the line widths. 


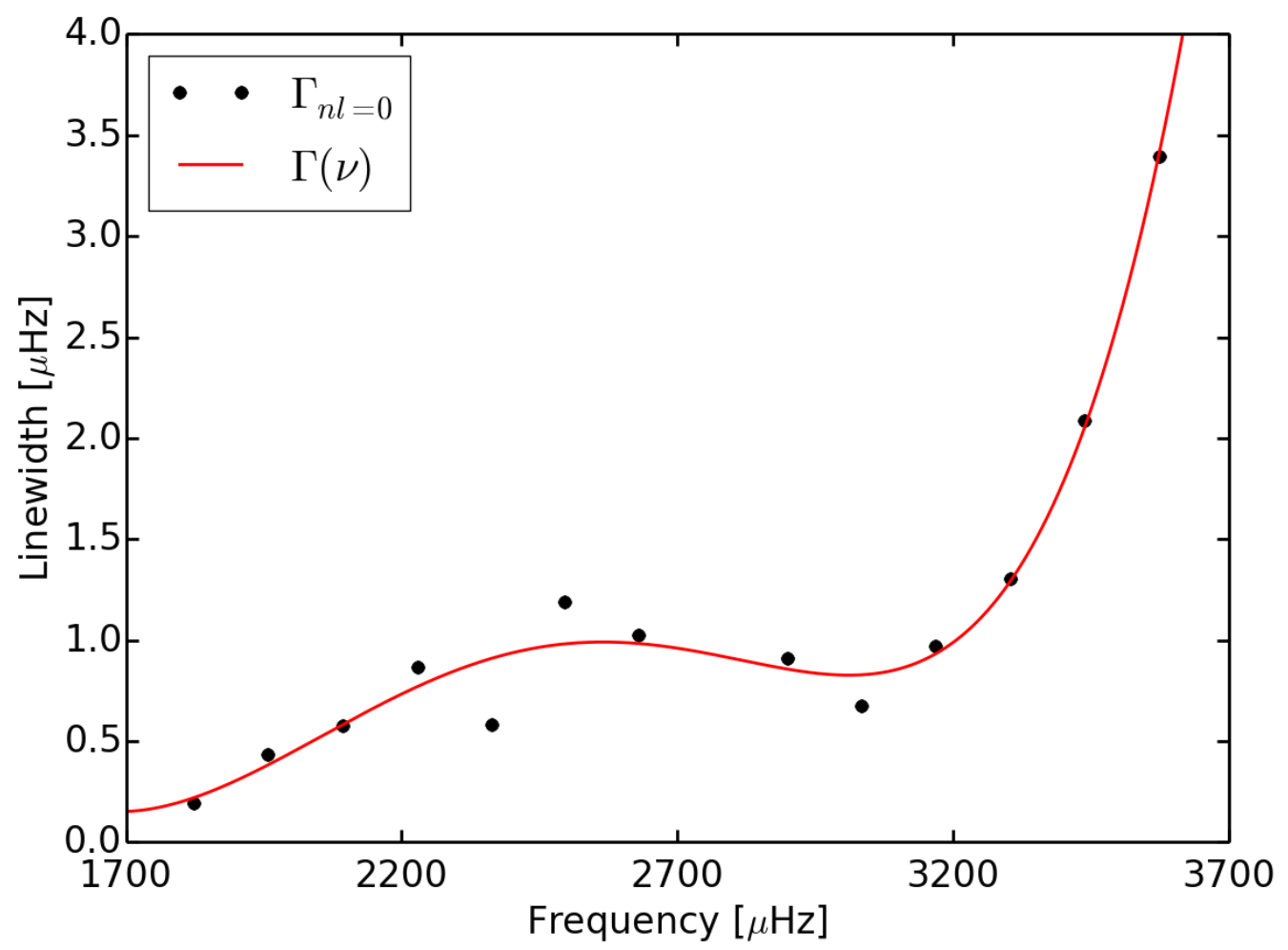

Figure 1.9: Linewidths of the solar $l=0$ modes (black) as a function of frequency, as observed by the Michelson Doppler Imager (J. Schou priv. com.). The points may be fit using a low order polynomial in frequency (red), to obtain a parameterized model of the linewidth as function of frequency.

Background noise: Observations of stellar flux contain a wide range of variability and depending on the purpose of the analysis, different parts of the spectrum may be considered noise. For peakbagging one typically defines noise as anything that interferes with the fitting of the oscillation peaks. The noise spectrum consists of three components: the frequency independent (white) photon noise; one frequency dependent component from brightness variations caused by granulation; and another frequency dependent term stemming from long-term variability such as activity or instrumental effects. Fig. 1.10 shows a spectrum of KIC006116048 in black, where a model for the background is shown in solid red and the individual background terms are shown in dashed red.

In the limit of large numbers of incident photons on the CCD the shot noise in the time series is distributed according to a Gaussian. This appears as a frequency independent level of noise in the power spectrum, and is typically modeled simply by a constant. The significance of the white noise relative to the oscillation modes can be mitigated by observing brighter targets or with long observation periods at high cadence.

At frequencies immediately below the $p$-mode envelope the granulation noise begins to dominate. This is caused by the granulation pattern on the stellar surface, which in turn is caused by convection cells reaching the photosphere. The auto-covariance of the granulation signal can be closely approximated by an exponentially decaying function 


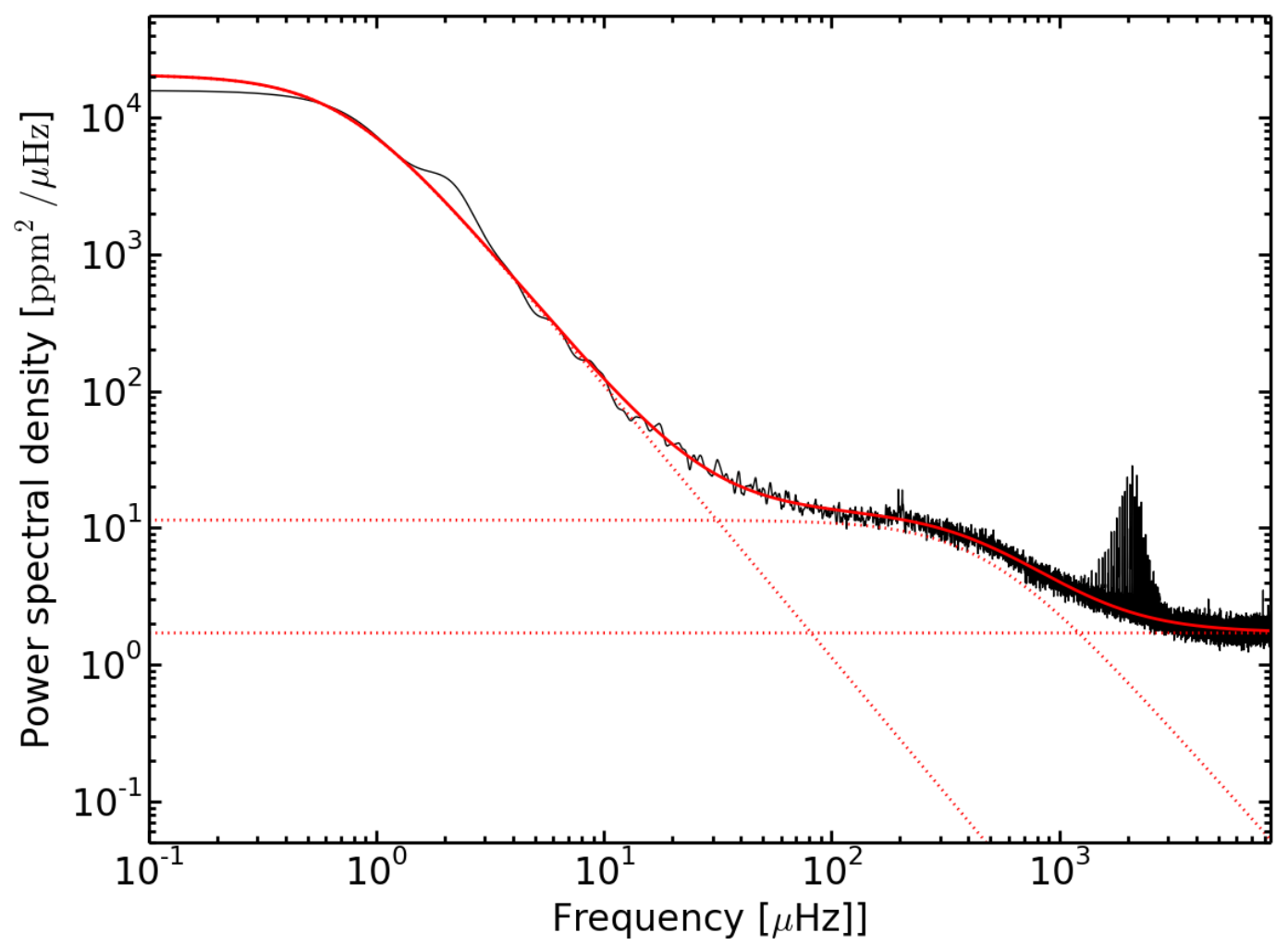

Figure 1.10: The smoothed power spectrum of KIC006116048 shown in black. The background model is shown in solid red, with the individual components of the model in dashed red.

with an $e$-folding time of $\tau$, which leads to a Lorentzian shape in the power spectrum with a width of $2 \pi \tau$ (Harvey 1985). This Lorentzian is centered around $v=0$, and its integral in frequency is proportional to the brightness variations in the time series caused by the granulation.

The last noise term at low frequencies stems from various sources. Like the granulation signal this is typically also modeled by a Lorentzian profile centered on $v=0$, however the characteristic timescale is much longer, on the order of the rotation period of the star or more. This noise term is therefore sometimes attributed to activity on the stellar surface, but may also contain the signatures of super-granulation (Vázquez Ramió et al. 2002). A significant component however, is likely also uncorrected instrumental noise, which has multiple characteristic frequencies.

The total background noise can be modeled by

$$
B(v)=\sum_{k=1}^{2} \frac{A_{k} \tau_{k}}{1+\left(2 \pi v \tau_{k}\right)^{\alpha_{k}}}+W
$$

where $W$ is the white noise and the sum is over the frequency dependent Lorentzian-like noise terms, with power $A_{k}$ and characteristic timescale $\tau_{k}$. The slopes $\alpha_{k}$ of the power decay with frequency is often simply set to $\alpha_{1}=\alpha_{2}=2$, as for Lorentzian profiles. However, there is currently some speculation that the slope of the profiles transitions to 
a steeper slope, i.e. $\alpha>2$ at high frequencies (Kallinger et al. 2010, Karoff et al. 2013, Kallinger et al. 2014). For the present work however, we have simply let $\alpha$ be a free parameter, which typically produces a values of $\alpha \approx 2$.

Maximum likelihood estimation: The noise in the power spectrum is distributed according to $\chi^{2}$ distribution with 2 degrees of freedom with a mean value equivalent to the limit spectrum (Abramowitz and Stegun 1972).

From the previous sections the complete model spectrum can be constructed by

$$
M(\boldsymbol{\theta}, v)=\sum_{n} \sum_{l=0}^{3} \sum_{m=-l}^{l} \frac{S_{n l} \mathscr{E}_{l m}(i)}{1+\left(2 / \Gamma_{n l m}\right)^{2}\left(v-v_{n l m}\right)^{2}}+\sum_{k=1}^{2} \frac{A_{k} \tau_{k}}{1+\left(2 \pi v \tau_{k}\right)^{\alpha_{k}}}+W
$$

where $\boldsymbol{\theta}=\left(v_{n l m}, \Gamma_{n l m}, S_{n l}, i, A_{k}, \tau_{k}, W\right)$ denotes the fitting parameters.

The probability that the power at a frequency $v_{j}$ takes a particular value $P_{j}$ is given as (Woodard 1984, Duvall and Harvey 1986, Appourchaux 2003)

$$
f_{j}\left(P_{j}\right)=\frac{1}{M\left(\boldsymbol{\theta}, v_{j}\right)} \exp \left(-\frac{P_{j}}{M\left(\boldsymbol{\theta}, v_{j}\right)}\right) .
$$

This allows us to calculate the probability of observing the power spectrum, given a particular model $M(\boldsymbol{\theta}, v)$. The objective is then to find the set of parameters $\boldsymbol{\theta}$ with the highest probability of explaining the observed power spectrum.

This is done by maximizing the likelihood $L$ given as the joint probability of Eq. 1.9 for all frequency bins $v_{j}$, and for a particular model $M\left(\theta, v_{j}\right)$. Typically the logarithm of the likelihood $\mathrm{L}$ is computed for better numerical stability, so that one must maximize

$$
\ln L(\boldsymbol{\theta})=\ln \prod_{j}^{N} f_{j}\left(P_{j}\right)=-\sum_{j}^{N} \ln M\left(\boldsymbol{\theta}, v_{j}\right)+\frac{P_{j}}{M\left(\boldsymbol{\theta}, v_{j}\right)} .
$$

It should be noted that this is not strictly correct if there are gaps in the time series since the frequency bins in the spectrum become correlated. The model spectrum should in principle be convolved with the spectral window function, after which the likelihood may be calculated. Stahn (2010) showed that at a high duty cycle this effect becomes minimal, and considering the duty cycle of the Kepler data $(\sim 91 \%)$ this correction was deemed unnecessary and time consuming in the computation of the joint probability.

Priors: Equation 1.10 easily lends itself to the application of prior information about the probability density function (PDF) of a given parameter. Provided the functional form of the PDF or an approximation of this is known it can be added to Eq. 1.10.

A prior biases one or more parameters toward a part of parameter space where one expects the true value of the parameter to lie. Obviously one should construct the prior carefully since it may incorrectly bias the relevant parameter, but may also skew correlated parameters. For very low $S / N$ data the PDF of the biased parameter will tend toward the prior. On the other hand, for very high $S / N$ the prior becomes almost meaningless if the data suggest something different. 
One obvious example of a prior is that of a uniform PDF, which is constant in some specified interval, and 0 everywhere else. This is sometimes called an ignorance prior, since it does not yield any information except by placing a boundary on the parameters.

Examples of priors relevant for peakbagging in asteroseismology are explored in Handberg and Campante (2011). These include: uniform priors on the frequencies, which may be used to avoid overlap between two closely spaced peaks such as the closely spaced $l=0$ mode and $l=2$ multiplet; a PDF which is uniform in logarithmic amplitude, which is typically used for scale parameters that span several orders of magnitude.

Lastly there is also the parameterizations that have been discussed above. These also act as priors, since they impose some functional form of the parameters based on some prior knowledge of their behavior.

Markov chain Monte Carlo sampling: To maximize $\ln L$ a suitable optimization algorithm must be chosen. The choice of algorithm is often motivated by the computation time of $\ln L$, as it may be necessary to explore a large section of parameter space and thus evaluate the likelihood many times. The aim is to find the global maximum in as few steps as possible. Such methods include the down-hill simplex method (Nelder and Mead 1965), gradient ascent/descent method, Powell's method (Powell 1964) and many more. However, such algorithms suffer from the possibility of getting stuck in a local maximum.

Alternatively one can randomly or pseudo-randomly sample the parameter space. This is the basis for Markov Chain Monte Carlo sampling. There are many different sampling techniques (see e.g. Metropolis et al. 1953, Hastings 1970, Geman and Geman 1984), however we opt to use the recently developed affine invariant sampling which tends to perform faster than other MCMC algorithms. The details of this sampler are described by Goodman and Weare (2010) and Foreman-Mackey et al. (2013). This method functions by invoking an ensemble of samplers, or 'walkers'. Each new position for a walker is a linear combination of its current position and that of another randomly chosen walker in the ensemble. This move in parameter space is then stretched by a randomly chosen factor. The only tuning parameters in this method are the number of walkers and the distribution from which the stretch factor is drawn. Both of these only impact the time it takes for the walkers to converge on the posterior distribution, and do not influence the result. This algorithm has been incorporated into the MCMC sampler package known as $\mathrm{EMCEE}^{9}$ for Python, which is used extensively in the following work.

The motivation for using this particular sampler is that because of the randomly chosen 'stretch move', which ensures that there is always some probability that the walkers will move out of a local maximum that they might have gotten stuck in. It is also easily implemented into the peakbagging process in a parallelized way, which is important given the size of the parameter space that must be sampled. The sampler yields robust estimates of the most likely fit solution, as well as estimates of the associated errors for even strongly correlated parameters in a high dimensionality parameter space. The down-side is that the sampler needs to run for an exceedingly long time in order to find the global maximum. The total run-time is the product of the time to evaluate $\ln L$, the number of walkers and the number of steps that they have to take to converge, which usually equates to several days.

\footnotetext{
${ }^{9} \mathrm{http}: / /$ dan.iel.fm/emcee/current/
} 

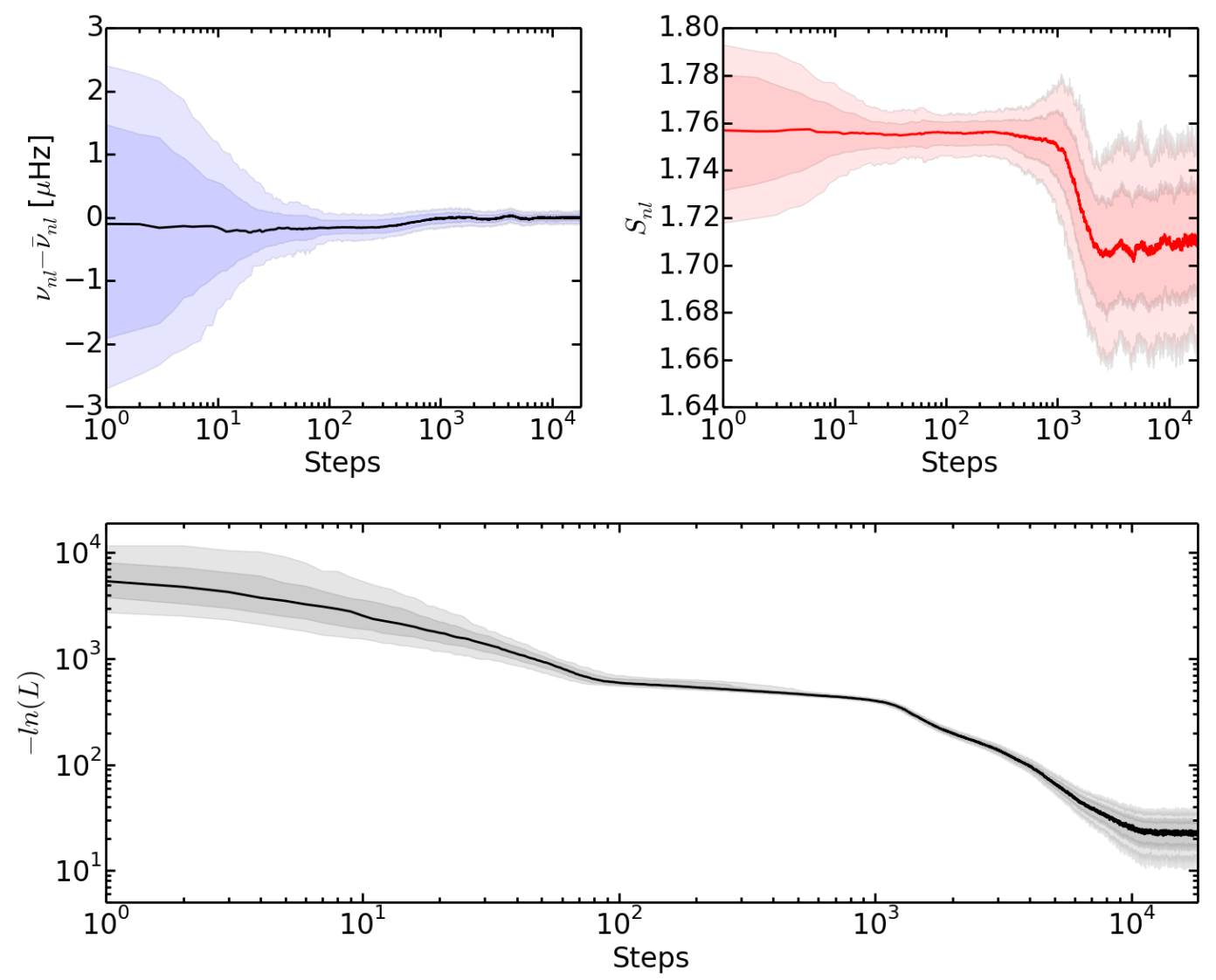

Figure 1.11: The positions of the walkers at each step in the chain are represented by contours containing the 95 (light shaded) and 68 (dark shaded) percentiles of the walker distribution, along each axis. The median of the distribution is shown in solid color. The top left frame shows the mean subtracted frequency of a mode in the oscillation spectrum of KIC006106415, along with the corresponding amplitude of the mode. The bottom frame shows the distribution of likelihoods in a similar fashion. Note that $-\ln L$ is shown on a logarithmic scale for clarity.

The majority of the samples taken early in the run, known as the 'burn-in' phase, are discarded since they represent very low likelihood locations in parameter space, and so are not meaningful when describing the posterior distribution. Once the walkers have converged on the global maximum their location in parameter space will be a good representation of the posterior distribution. The mode of the marginalized posterior distribution of a given parameter represents the best-fit value. Often the median and the 16th and 84th percentiles of the marginalized posterior are used as representative values. In the case of a Gaussian posterior these values would represent the mean and the $1 \sigma$ confidence interval.

It can be difficult to estimate when the MCMC chains have converged. Several metrics and methods exist that attempt to quantify convergence (reviewed by Cowles and Carlin 1996). However, it is often most instructive to simply look at the walker positions as a function of step number (called the trace). The top two frames in Fig. 1.11 show the traces of two parameters of a peakbagging run of KIC006106415. The density of walkers 
is represented by the contour regions, with the median location of the walkers shown in solid color. After an initial period of wandering through parameter space the walkers settle into a localized region and stay there, indicating that this is the global maximum.

Similarly, one can also estimate convergence by the likelihood of each walker position as a function of step through parameter space. This is shown in the bottom panel of Fig. 1.11. The likelihood should go asymptotically toward the global maximum. Once the change over time becomes approximately zero the chains are likely burnt in.

\subsection{Applications of rotation measurements}

The measurements of rotation have applications mainly in three areas of astrophysics which are briefly discussed in the following section. These include the study of how rotation evolves over time, which in turn may be applied to using rotation as an age estimator when other methods are not applicable. Rotation also influences the mixing of elements inside stars, which affects their nuclear burning rates and is particularly relevant for the hot, massive stars that tend to be fast rotators. For stars with solar-like magnetic activity, differential rotation is thought to play a major role in the generation of global magnetic fields through the stellar dynamo.

\subsubsection{Gyrochronology}

In Fig. 1.1 we saw that the median rotation rate of clusters past the ZAMS steadily decreases. This was also visible in Fig. 1.2 where the main rotation sequence of M48 is located at longer periods than that of the Pleiades. Skumanich (1972) initially measured this spin-down over time through observations of the Pleiades and Hyades clusters, and the Ursa Major group. The rotation rates of the clusters were found to decrease as $\Omega \propto A^{-1 / 2}$, where $A$ is the age of the star. Based on a relation between the magnetic field strength and the stellar rotation rates by Mestel (1984), Kawaler (1988) determined that this was consistent with a spin-down caused by the wind driven mass-loss. Furthermore, Fig. 1.2 also showed that the rotation period of the star on the main rotation sequence is a function of mass. This suggests that the rotation period and mass of a star can be used as a means of estimating its age, often called 'gyrochronology'. Stars that are known to be coeval with nearby stars, such as other cluster members, can be dated quite precisely through isochrone fitting in a color-magnitude diagram. Field stars on the other hand do not typically have companion objects that can be used as references. Moreover, on the MS their fundamental properties, e.g., mass, temperature, luminosity, radius etc. hardly change. Any observable parameters that measure these quantities therefore will not change remarkably either, making age estimates of a single star extremely difficult ${ }^{10}$. Relative to the structural properties of the star the rotation rate changes dramatically. A relation between the mass, rotation period, and age would therefore be a particularly useful tool for determining the age of a field star. The rotation period and color index of the

\footnotetext{
${ }^{10}$ Only recently through the use of asteroseismology, has it become possible to precisely determine the age of a star. This technique requires very high quality data however, and is also not applicable to very active stars.
} 


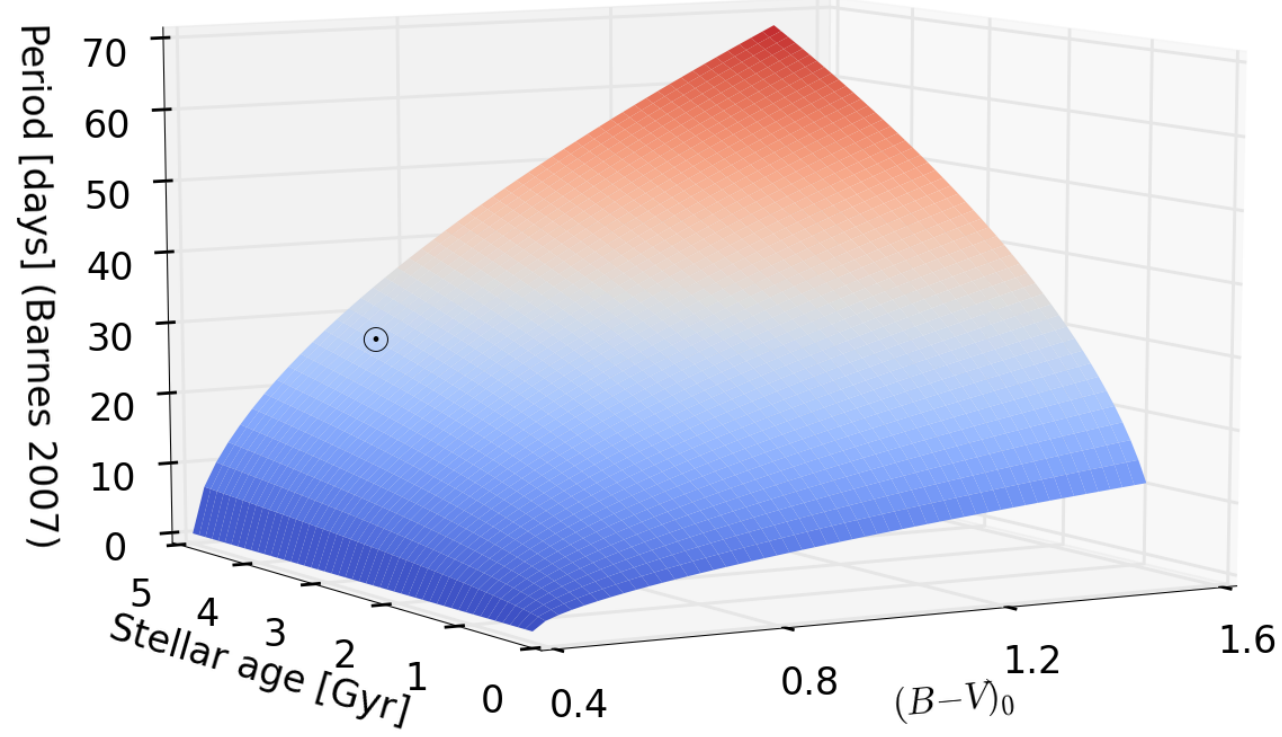

Figure 1.12: The period-mass-age plane (Eq. 1.11) for the relation by Barnes (2003). In this study solar values and those from several young open clusters were used as a calibrators.

star are very easily measurable, and so through these two quantities it would be possible to determine the stellar age.

Typically the gyrochronology relation takes the form of an empirically determined power law (Barnes 2003, 2007, Mamajek and Hillenbrand 2008, Angus et al. 2015)

$$
P=A^{n} a\left((B-V)_{0}-c\right)^{b},
$$

where $P$ is the mean rotation period of the star, $A$ is the stellar age, and $(B-V)_{0}$ is the extinction corrected $\mathrm{B}-\mathrm{V}$ color index acting as a proxy for the stellar mass. The remaining variables $n, a$, and $b$ are calibration constants. Figure 1.12 shows this relation using the constants derived by Barnes (2007), which were obtained by fitting to the age and rotation rates of the Sun and several young cluster stars. Finding the precise value of these calibration constants has however proven to be difficult and a subject of much debate (Karoff et al. 2013, Metcalfe et al. 2014, García et al. 2014, see the above references as well as). It should be stressed, that this relation is purely empirical, and so the form of the relation and the calibration constants are not physically motivated.

Cluster stars have so far been the favored means of calibrating these relations because of their precise age measurements. Along with color photometry and rotation period measurements, the above relation may be calibrated. Unfortunately the majority of cluster ages are only a few hundreds of Myr, and so are poor calibrators for stars of similar age to the Sun. The oldest cluster that has been used so far for this calibration is NGC6819 
(Meibom et al. 2015), which is only 2.5Gyr. Moreover, the applicability of such a simple relation for evolved MS stars has recently been called into question (van Saders et al. 2016, see also Sect. 4.5). Using independent measures of stellar age from asteroseismology, these stars appear to rotate too fast, compared to what is predicted by a simple spin-down law like Eq. 1.11.

\subsubsection{Impact of rotation on stellar evolution}

Rotation affects the structure of a star and therefore its evolution. The effect is first and foremost to introduce a centripetal force term into the equation of hydrostatic equilibrium. For slowly rotating stars like the Sun this is typically neglected (Maeder and Meynet 2000). The effect of rotation can be gauged by the ratio of the centripetal acceleration and the gravitational acceleration; for the Sun this ratio is on the order of $10^{-5}$, whereas for the larger, more massive and much faster rotating star Vega this ratio is on the order 0.5.

For these massive, fast rotating stars the effects of rotation become visible primarily through the so-called gravity-darkening. By the von Zeipel theorem (von Zeipel 1924) the local radiative flux throughout a star is proportional to the local effective gravity. Because of the centripetal acceleration the local gravity at the equator must be lower than at the poles, and so the radiative flux is higher at the poles than at the equator.

For a massive star the gravity-darkening has two main effects. First it induces a poleward meridional flow at the surface of the star, which has a return flow deep in the interior. This acts as a form of chemical mixing, where remnants from fusion burning in the core are replaced by fresh material from closer to the surface, prolonging the MS lifetime of these stars (see, e.g., Brandt and Huang 2015). Secondly, for a star with gravity-darkening, the viewing angle of the observer now becomes an important factor when determining the stellar brightness. Because of the intrinsic random scatter in the inclination angle of the stars relative to the observer, this will lead to increased scatter in the observed magnitudes of hot stars. These effects have important implications when attempting to estimate the ages of young clusters by way of the color-magnitude diagram (CMD). It increases the spread in the CMD at the MS turn-off, which is an important reference point for the age determination of clusters (Meynet et al. 2009). Clearly this impacts studies that rely on precise cluster ages, like gyrochronology.

\subsubsection{Solar and stellar dynamos}

The Sun is a magnetic star. It is observed to go through an 11 year cycle of high and low magnetic activity (often simply called solar maximum and minimum), where the global dipole changes polarity from cycle to cycle. Despite a wealth of observational constraints, there is no consensus on the physical causes behind the solar dynamo. A simplified picture of the magnetic cycle is as follows: During solar maximum the strength of the poloidal magnetic field is at a minimum, and it is thought that the toroidal component of magnetic field is at its maximum (Charbonneau 2010). At this point the polarity of the poloidal field is reversed. During this time large numbers of active regions, sunspots and other magnetic phenomena are present on the solar surface. This is followed by a period of relative inactivity, where the poloidal field grows in strength. Once the next maximum 
is reached the polarity of the poloidal field is once again reversed, and the process starts over.

Many models currently exist that attempt to describe the solar dynamo (see the review by Charbonneau 2010). Each one differs in the detailed description of how the transition between the poloidally and toroidally dominated field structures, however most of models rely on differential rotation to act as a source term for one or both of the components. This is either in the form of the surface shear layer or tachocline (Miesch 2005).

Observations of magnetic fields on other stars have allowed for the extension of dynamo models into new regimes of parameter space, such as varying magnetic field strength, field configuration, rotation rate, as well as thin convection versus full convection. Observations range from simple estimates of spot coverage from, e.g., photometric time series and Doppler imaging to activity index measurements from dedicated long period surveys (Wilson 1968, Baliunas et al. 1995, Karoff et al. 2013). Such long period surveys show both stars that have cyclic and secular variations in activity, as well as some that do not show any variation at all. Böhm-Vitense (2007) found that a selection of the stars with periodic variability in activity fell into two distinct sequences of correlation between cycle period and rotation period. This was interpreted as the presence of two different dynamos operating in the two groups of stars as also suggested by Vaughan and Preston (1980) and Durney et al. (1981). Detailed inversions of the surface magnetic field structure with spectropolarimetry have also shown different behaviors of the magnetic dynamos of other stars, from non-cyclic behavior (Jeffers et al. 2011, Donati et al. 2003) to the same field reversal behavior as the Sun (Donati et al. 2008, Fares et al. 2009).

These studies all measure the physical effects of stellar dynamos which are manifested at the surface. They are limited to speculation about the root cause of the dynamos based only on these surface effects and the global physical parameters of the star. Clearly measuring the internal angular momentum distribution can help in this regard, since it will be possible to test the effects of differential rotation in dynamo models, to see if they are able to reproduce similar levels of magnetic activity given the observed surface activity. However, so far this has not been done for any stars on the MS except the Sun. 



\section{Paper I - Rotation periods of 12000 main-sequence Kepler stars}

This chapter has been published in 2013 in A\&A vol. 557, page L10. The work was carried out and written by myself, under the supervision of L. Gizon, H. Schunker from the Max Planck Institute for Solar System Research and in collaboration with C. Karoff from Aarhus University, Denmark.

\subsection{Summary of Paper I}

We aim to measure the starspot rotation periods of active stars in the Kepler field as a function of spectral type and to extend reliable rotation measurements from F-, G-, and K-type to M-type stars. Using the Lomb-Scargle periodogram we searched more than 150000 stellar light curves for periodic brightness variations. We analyzed periods between 1 and 30 days in eight consecutive Kepler quarters, where 30 days is an estimated maximum for the validity of the PDC_MAP data correction pipeline. We selected stable rotation periods, i.e., periods that do not vary from the median by more than one day in at least six of the eight quarters. We averaged the periods for each stellar spectral class according to B-V color and compared the results to archival $v \sin i$ data, using stellar radii estimates from the Kepler Input Catalog. We report on the stable starspot rotation periods of 12151 Kepler stars. We find good agreement between starspot velocities and $v \sin i$ data for all F-, G- and early K-type stars. The 795 M-type stars in our sample have a median rotation period of 15.4 days. We find an excess of M-type stars with periods less than 7.5 days that are potentially fast-rotating and fully convective. Measuring photometric variability in multiple Kepler quarters appears to be a straightforward and reliable way to determine the rotation periods of a large sample of active stars, including late-type stars.

\subsection{Introduction}

Measuring stellar rotation as a function of age and mass is essential to studies of stellar evolution (Maeder 2009) and stellar dynamos (Böhm-Vitense 2007, Reiners et al. 2012). Rotation can be measured at the surface of individual stars using either spectroscopy (e.g., Royer et al. 2004) or periodic variations in photometric light curves due to the presence of starspots (Mosser et al. 2009). On the Sun, sunspots and plage regions modulate the solar irradiance with periods close to the solar rotation period. Brightness variations are also seen in other stars and are commonly attributed to the presence of magnetic activity 
in the case of main-sequence cool dwarfs (e.g. Berdyugina 2005). Sunspots and solar active regions have lifetimes of days to weeks (rarely months) (Solanki 2003) and are reasonably good tracers of solar surface rotation at low latitudes. Starspots have been observed to persist for even longer periods (e.g. Strassmeier 2009), and they appear at high latitudes as well.

The Kepler mission (Borucki et al. 2010) has been monitoring the light emitted by more than $10^{5}$ stars since its launch in 2009. Treating such an enormous number of stars obviously demands an automated approach. In this paper we present the results of a straightforward automated method for analyzing Kepler time series and detecting amplitude modulation due to stellar activity, with the aim of determining stellar rotation periods.

\subsection{Measuring stellar rotation}

\subsubsection{Kepler photometry}

We used white light time series with a cadence of 29.42 minutes from the NASA Kepler satellite. The data are released in segments of $\sim 90$ days (quarters) through the Mikulski Archive for Space Telescopes ${ }^{1}$ for a total sample of stars currently numbering $\sim 190000$. We used quarters 2 through 9 (two years of observations in total). The data was processed for cosmic rays and flat fielding prior to release. We used the version of the data that was corrected by the PDC_MAP pipeline (Smith et al. 2012). The PDC_MAP correction attempts to detect and remove systematic trends and instrumental effects, which are common to a large set of adjacent stars on the photometer. In addition, we used the most recent data from the msMAP correction pipeline (Thompson et al. 2013, where 'ms' stands for multi-scale) to check for consistency with PDC_MAP.

From an initial sample of 192668 stars, we discarded targets that are known eclipsing binaries (Matijevič et al. 2012), planet host stars as well as planet candidate host stars, and Kepler objects of interest (all lists are available through the MAST portal). We do this to reduce the possibility of false positive detections, since these types of variability may be mistaken for transits of starspots.

\subsubsection{Detecting rotation periods}

Provided active regions or starspots are present over several rotations of the star, a peak will appear in the periodogram of the time series. Assuming starspots trace surface rotation, this provides a way to measure the rotation rate of the star at the (average) latitude of the starspots. Simulations by Nielsen and Karoff (2012) show that selecting the peak of maximum power is a suitable method for recovering the stellar rotation period.

We analyzed the Kepler observations as follows:

1. We compute a Lomb-Scargle (LS) periodogram (see Frandsen et al. 1995) for each star in each quarter for periods between 1 and 100 days, using PDC_MAP data.

2. We find the peak of maximum power in this period range and record its period.

\footnotetext{
${ }^{1} \mathrm{http}: / /$ archive.stsci.edu/kepler/
} 
3. If the period of the peak falls between 1 and 30 days, we consider it due to stellar variability and not instrumental effects.

4. The peak height must be at least four times greater than the white noise estimated from the root mean square (RMS) of the time series (Kjeldsen and Bedding 1995).

The lower bound in periods of one day is set to avoid the hot g-mode pulsators with frequencies of a few cycles per day (see Aerts et al. 2010, chap. 2). Some contamination from g-mode pulsations is expected for F- or earlier-type stars; however, we have not investigated how to automatically differentiate these pulsations from stellar activity variability. The upper bound in period of 30 days is the estimated limit for which the PDC_MAP pipeline does not overcorrect the light curve, to the extent that it completely removes the intrinsic stellar signal (Thompson et al. 2013). We calculated periods up to 100 days to ensure that any peak found below 30 days is not a potential side lobe of a dominating long-term trend ( $>30$ days).

\subsubsection{Selecting stable rotation periods}

Further, we require the that the measured periods are stable over several Kepler quarters. Specifically,

5. we determine the median value of the measured periods over all eight quarters;

6. we select stars for which the median absolute deviation (MAD) of the measured periods is less than one day, i.e., MAD $<1$ day ; The MAD is defined as MAD = $\left\langle\left|P_{i}-\left\langle P_{i}\right\rangle\right|\right\rangle$ (where \langle\rangle is the median).

7. From these, we select stars with six or more (out of eight) measured periods within 2 MAD of the median period;

8. we repeat this method using the msMAP data and flag stars that do not satisfy the above criteria.

We use the MAD since it is less sensitive to outliers than the standard deviation (Hoaglin et al. 2000). Requiring that a particular variation for a star is visible in multiple quarters reduces the risk of the detection coming from low-frequency noise from, say, instrumental effects. The LS periodogram is calculated at $\sim 1300$ linearly spaced frequencies between $1.2 \times 10^{-2} \mathrm{mHz}$ and $3.9 \times 10^{-4} \mathrm{mHz}\left(0.03 \mathrm{~d}^{-1}\right.$ and $\left.1 \mathrm{~d}^{-1}\right)$. The MAD limit of one day (point 6), along with the signal attenuation introduced by the PDC_MAP correction, leads to a selection bias towards stars with shorter rotation periods. Examples of periods detected for three stars are shown in Fig. 2.1.

We applied the scaling relation by Kjeldsen and Bedding (2011) to find timescales for p-mode pulsations in cool main sequence stars and red giants. We found that stars with $\log g \lesssim 2$ have pulsation periods that can potentially overlap with the range investigated in this work. We opted for a conservative approach and discarded stars with $\log g<3.4$ to remove red giants from the sample. Following the scaling relation, the main sequence stars were found to have pulsation timescales from minutes to hours, far below our lower period limit. Once all the above criteria are met, the rotation period, $P_{\text {rot }}$, is defined as the 


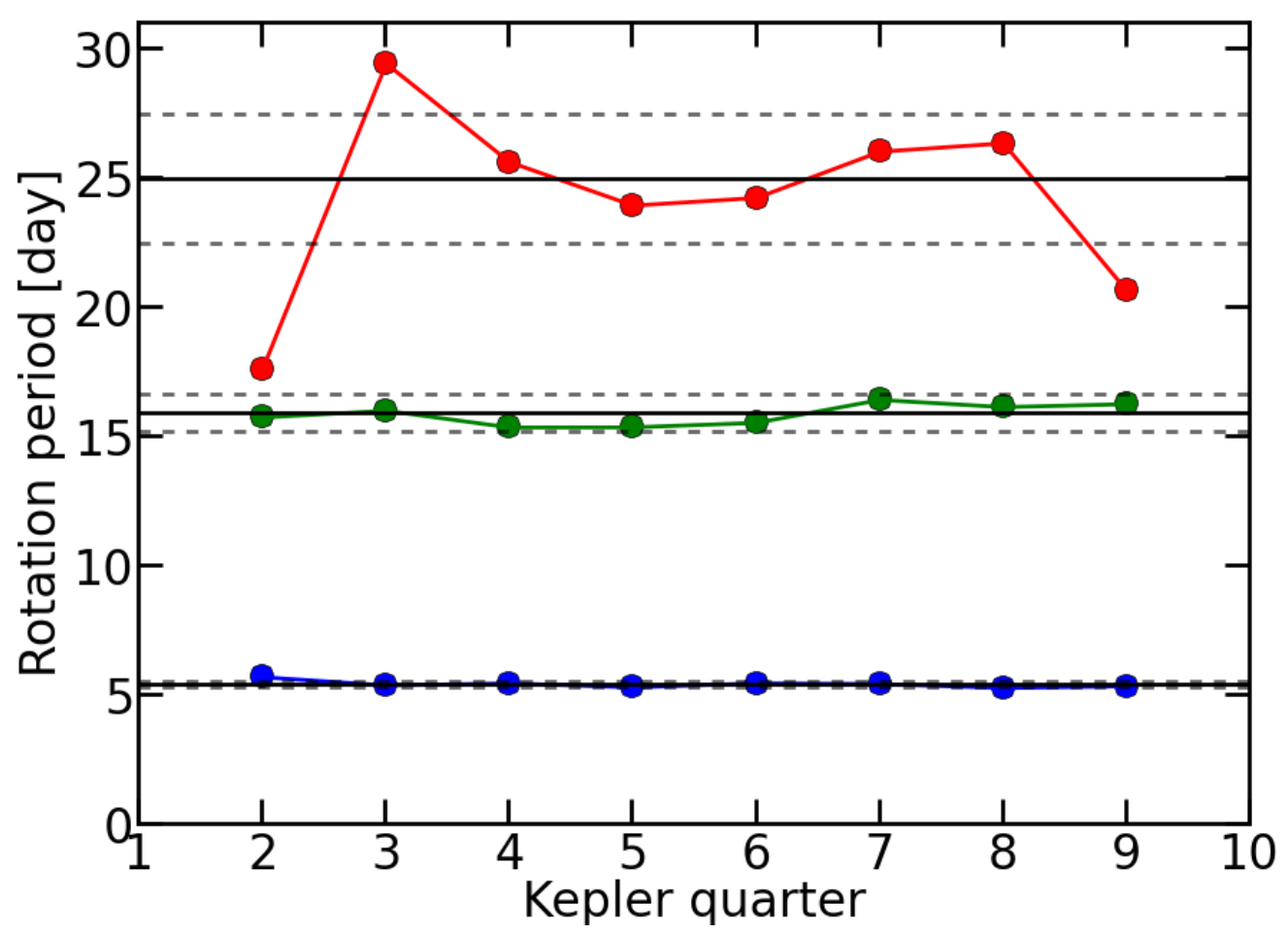

Figure 2.1: Measured periods in each quarter of observation for three Kepler stars (red, green and blue). The solid line is the median period over all quarters for each star. The dashed horizontal lines indicate two median absolute deviations (MAD) from the median period. The long period target (red) is discarded by the algorithm due to high scatter in the period measurements $(\mathrm{MAD}=1.26$ days). The green and blue target are examples of stars that meet the selection criteria.

median of the valid periods. This selection process leaves us with 12151 stars out of the original sample of 192668 . When using the msMAP data, we find that $\sim 80 \%$ of these stars satisfy the above criteria as well. Of these, $0.9 \%$ differ from the PDC_MAP results by more than one resolution element, predominantly because the msMAP data shows the first harmonic instead of the fundamental period of the variability. The msMAP pipeline treats the long periods $\left(P_{\text {rot }} \lesssim 15\right.$ days) differently than the PDC_MAP (see Thompson et al. 2013).

The results for all 12151 stars are shown in Table 1, which is provided as online material through the CDS. Column 1 gives the Kepler Input Catalog (KIC) name of the star, cols. 2 and 3 are the rotation period and scatter (MAD), and cols. 4 to 8 give the $g-r$ color, $E(B-V)$, radius, $\log g$, and $T_{\text {eff }}$, respectively, all of which are KIC values. Column 9 is a flag indicating whether each msMAP-corrected data set satisfies the criteria of section 2.3. Column 10 gives the msMAP period of the stars where we find rotation rates from the two data sets that differ by more than one resolution element. 


\subsection{Consistency with $v \sin i$ measurements}

We performed a rudimentary spectral classification of the stars based on their $B-V$ color indices. The KIC provides $g-r$ values that we converted to $B-V$ using the relation $B-V=0.98(g-r)+0.22$ given by Jester et al. (2005) for stars with R-I color indices $<1.15$. The $B-V$ values are dereddened using the $E(B-V)$ values from the KIC (see Brown et al. 2011, for details on their derivation). The calculated $B-V$ colors are mapped to a spectral type as per Gray (2005, Appendix B).

We compared the periods found in this work with $v \sin i$ values compiled by Glebocki and Gnacinski (2005). This list contains $v \sin i$ values and spectral types for $\sim 30000$ cluster stars almost isotropically distributed in galactic coordinates. Using our approximate spectral classification we compared the median equatorial velocities of this sample with our results from the Kepler targets. From the $v \sin i$ sample we select stars with apparent $\mathrm{V}$ magnitude from 6 to 15, roughly equivalent to the magnitude range of the Kepler targets. We discard any stars from the $v \sin i$ sample that have been ambiguously labeled as 'uncertain'. Lastly, we select only dwarf stars since this is the main constituent of the stars selected by our method, reducing the $v \sin i$ sample to be comparable in number to our Kepler target list.

For each spectral type (s.t.) in our sample of Kepler targets we calculate the median equatorial rotational velocity by $\bar{v}($ s.t. $)=2 \pi\left\langle R_{\mathrm{KIC}} / P_{\text {rot }}\right\rangle$ where \langle\rangle denotes the median over stars with spectral type s.t., $R_{\mathrm{KIC}}$ is the KIC stellar radius, and $P_{\text {rot }}$ is the rotation period determined by our algorithm. The KIC radii are notoriously bad estimates in some cases; however, by comparing these with characteristic radii given in Gray (2005, Appendix B) we find that the median values in the region of $\mathrm{F} 0$ to $\mathrm{K} 0$ agree within $\sim 15 \%$. Outside this range of spectral types the radii are initially overestimated, but become strongly underestimated for A-type stars and earlier.

Since we have such a large statistical sample of $v \sin i$ measurements within each spectral type, a random distribution of inclinations of rotation axes gives $\langle\sin i\rangle=\pi / 4$ (e.g., Gray 2005). Therefore the median equatorial velocity for a given spectral type can be approximated by $(4 / \pi)\langle v \sin i\rangle$, which is directly comparable to the $\bar{v}($ s.t. $)$ computed for the Kepler targets.

Panel A in Fig. 2.2 shows clear agreement, from late A-type to early G-type stars, between the median equatorial velocities of the $v \sin i$ sample and those we derived from our measured Kepler rotation periods. For additional comparison we included the $v \sin i$ measurements from Reiners and Mohanty (2012), where the horizontal bar represents 201 stars with velocities below $4 \mathrm{~km} / \mathrm{s}$. Panel B shows our measured rotation periods $P_{\text {rot }}$, as a function of spectral type, which we used to calculate the equatorial velocities. We also calculated the median periods of the Kepler stars found in Debosscher et al. (2011) and McQuillan et al. (2013) that are present in our sample. These are shown in comparison to our results in panel $\mathrm{C}$. We find that $\sim 96 \%$ and $\sim 97 \%$ of their measured rotation rates fall within one frequency resolution element $\left(1 / 90 \mathrm{~d}^{-1}\right)$ of our values for the corresponding stars. 

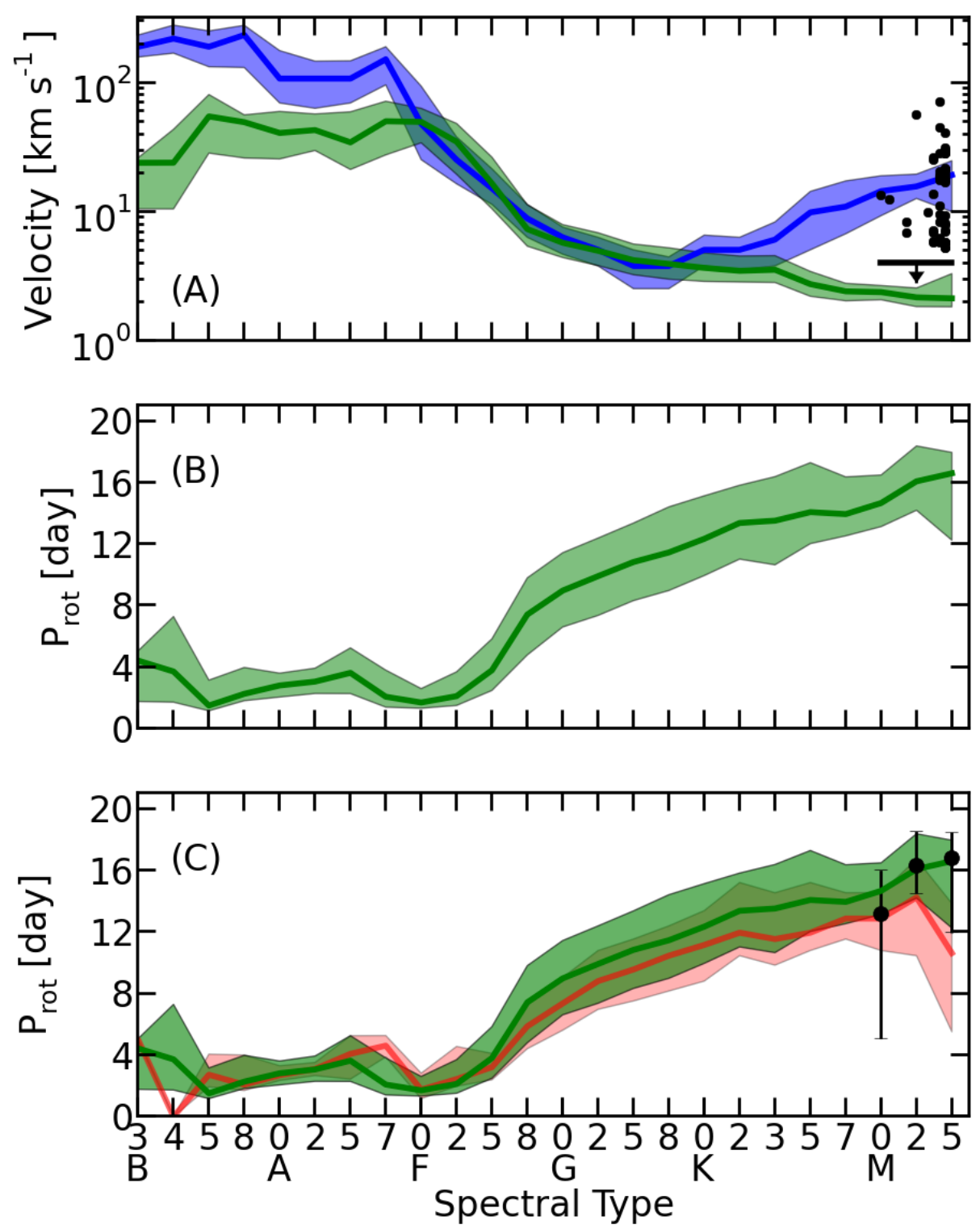

Figure 2.2: Panel A: The blue curve is the median equatorial velocity $(4 / \pi)\langle v \sin i\rangle$ for each spectral type from Glebocki and Gnacinski (2005). The green curve shows the equatorial velocity of the Kepler targets, $\bar{v}$ (s.t.), derived from the measured rotation periods and the KIC radii. The black points show measurements by Reiners and Mohanty (2012). In this sample 201 stars have an upper $v \sin i$ limit of $4 \mathrm{~km} / \mathrm{s}$ (due to instrumental limitations), these stars are represented by the solid bar. Panel B: The rotation periods $P_{\text {rot }}$ of the stars in our sample, averaged within each spectral type. Panel C: The same as panel $\mathrm{B}$, but for comparison we show the median of the rotation periods measured by McQuillan et al. (2013) (black points with errorbars), for the stars overlapping with our sample. Similarly, the red curve shows the median of the rotation periods found by Debosscher et al. (2011). Shaded areas and error bars span the upper and lower $34^{\text {th }}$ percentile values from the median. 


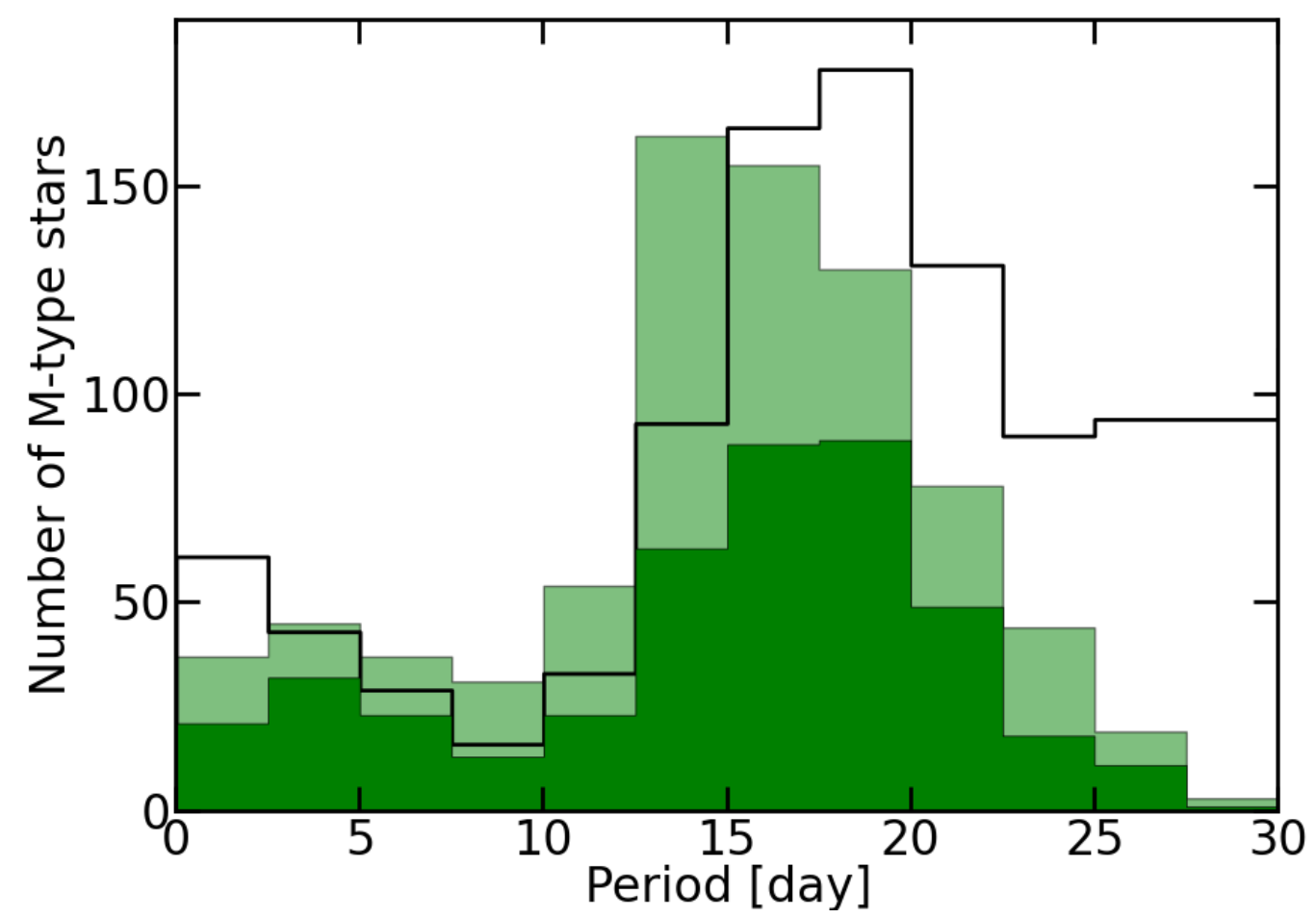

Figure 2.3: The light green histogram shows the distribution of rotation periods for the 795 M-type stars in our sample (median, 15.4 days). For comparison the black line shows the results by McQuillan et al. (2013) for periods less than 30 days. The dark-green histogram shows the distribution of rotation periods measured by McQuillan et al. (2013) for the stars in common with our sample.

\subsection{Rotation of late type stars}

Owing to their small radius and long rotation period, the $v \sin i$ measurements of late type stars are limited by spectral resolution. The $v \sin i$ measurements of Reiners and Mohanty (2012) have a lower limit at $\sim 4 \mathrm{~km} / \mathrm{s}$. The KIC radii are overestimated for stars later than K0 so our velocities are therefore upper limits, but we still systematically find rotation slower than $\sim 4 \mathrm{~km} / \mathrm{s}$. This places our measurements at or below the lower limit for spectroscopically determining rotation for these types of stars.

The late M-type stars are of particular interest since they represent the transition from solar-like convective envelopes to fully convective interiors. Figure 2.3 shows the distributions of stars that have been classified as M-type, found in this work and those by McQuillan et al. (2013). The median of the distribution in our sample is 15.4 days. There appears to be good agreement with the result of McQuillan et al. (2013). We note an excess of fast rotators with $P<7.5$ days. The study by Reiners and Mohanty (2012) shows that the number of magnetically active M-type dwarfs increases approximately after spectral subtype M3, which appears correlated with an increase in the number of fast rotators (see top panel of Fig. 2.2), likely marking the transition to full convection. Our sample contains 795 M-type stars, where $\sim 15 \%$ have periods less than 7.5 days; i.e., these are potentially fast-rotating, fully convective M-type dwarfs, such as those found by Reiners 
and Mohanty (2012). As a result of the KIC $\log g$ having errors of approximately \pm 0.4 dex (Brown et al. 2011), the sample probably still contains some cool giants. A visual inspection of the power spectra of the M-type stars, with periods $P<7.5$ days, indicated that 3 out of 119 stars had p-mode pulsations that could potentially be misidentified as periodic activity. Thus the contamination by p-mode pulsations appears to be negligible. A more rigorous determination of $\log g$ and spectral type for these stars is required to distinguish stellar evolutionary stages. However, even a fraction of our sample of M-type dwarfs still significantly complements the existing literature on this critical region for understanding differences in stellar dynamos.

\subsection{Conclusions}

We developed a straightforward, automated method for detecting stable rotation periods for a large sample of Kepler targets. A total of 12151 stars ranging from spectral type B3 to M5 all show recurring periods in at least six of the eight quarters of analyzed Kepler data. This, along with the requirement that the stars in question have a maximum MAD of one day, are empirically determined criteria. Using the KIC stellar radii, we found very good agreement between the equatorial rotational velocities derived from the Kepler rotation periods and independent $v \sin i$ measurements for F0 to K0 type stars. For later type stars we find an inconsistency with the Glebocki and Gnacinski (2005) catalog. This is due to an age difference between the two samples, since the Glebocki and Gnacinski (2005) catalog mainly consists of young open cluster stars. However, our results for Mtype stars agree well with those reported by Reiners and Mohanty (2012) and McQuillan et al. (2013). The study by Debosscher et al. (2011) analyzed a different set of Kepler data, but we still find good agreement with the stars overlapping with our sample.

We have primarily studied stars that have long-lived stellar activity signatures. We tested our method on solar-disk integrated light at solar maximum (January 2001 to March 2003), observed by the VIRGO instrument aboard SOHO (Frohlich et al. 1997). The VIRGO green channel data were divided into 90-day segments, and we applied the method described in Sect. 2.3. The stability criterion was not met due to a period scatter of MAD = 3.7 days. Analysis of other segments of VIRGO data from 1995 to 2013 also resulted in rejection. This shows that stars with solar-like (low) activity are rejected from our sample; for such stars, rotation period measurements are too noisy and not stable enough over time. A further bias is the upper limit of $P \leq 30$ days chosen because the PDC_MAP does not yield reliable corrections for longer periods. An obvious improvement to our analysis would be to investigate ways of increasing this upper limit, for a complete view of slow rotators.

As expected, we found that hot stars rotate faster than their cooler counterparts (Barnes 2003, Kraft 1970). The rotation periods of hot stars should be treated with some caution since they may be false positives from g-mode pulsations. Nevertheless, our analysis method detects periodic variations in brightness that satisfy the selection criteria. The measured variability is not necessarily of magnetic origin, but could arise from, for example, chemical surface inhomogeneities in chemically peculiar hot stars. Chemical spots can produce photometric variability that traces the stellar rotation (Wraight et al. 2012, Paunzen et al. 2013). The short periods measured for the early spectral types (see Fig. 
2.2) in our sample are therefore not contradictory to the expected fast rotation of hot stars (Royer et al. 2004).

This work is only one step toward characterizing of the rotation of stars in the Kepler field. Kepler photometry is proving very useful in adequately sampling the slow rotation rates of cool, faint stars. Future work will include detailed starspot modeling in order to measure latitudinal differential rotation (Reinhold and Reiners 2013) and asteroseismology (e.g., Deheuvels et al. 2012) to infer internal differential rotation. The Kepler observations offer unique possibilities for calibrating the mass-age-color relations in gyrochronology (Skumanich 1972, Barnes 2007) and exploring the close relation between stellar rotation and activity cycles. 



\section{Paper II - Rotational splitting as a function of mode frequency for six Sun-like stars}

This chapter has been published in 2014 in A\&A vol. 568, page L12. The work was carried out and written by myself, under the supervision of L. Gizon, H. Schunker and J. Schou from the Max Planck Institute for Solar System Research.

\subsection{Summary of Paper II}

Asteroseismology offers the prospect of constraining differential rotation in Sun-like stars. Here we have identified six high signal-to-noise main-sequence Sun-like stars in the $\mathrm{Ke}$ pler field, which all have visible signs of rotational splitting of their $p$-mode frequencies. For each star, we extract the rotational frequency splitting and inclination angle from separate mode sets (adjacent modes with $l=2,0$, and 1) spanning the $p$-mode envelope. We use a Markov chain Monte Carlo method to obtain the best fit and errors associated with each parameter. We are able to make independent measurements of rotational splittings of $\sim 8$ radial orders for each star. For all six stars, the measured splittings are consistent with uniform rotation, allowing us to exclude large radial differential rotation. This work opens the possibility of constraining internal rotation of Sun-like stars.

\subsection{Introduction}

Until recently, measuring the rotation of a star other than the Sun has been restricted to measuring the rotation rate at, or near, the photosphere. Techniques such as spectral line broadening obtain the projected rotational velocity $v \sin i$ (Kraft 1970, Gray 2005), where $i$ is the inclination of the stellar rotation axis with respect to the line of sight. However, this is difficult for slowly rotating stars (e.g., Reiners et al. 2012) and a measurement is fundamentally ambiguous because of the often unknown inclination. An alternative to this approach is to analyze photometric light curves for signs of active regions crossing the stellar disk (Nielsen et al. 2013, Reinhold and Reiners 2013, McQuillan et al. 2014). If enough crossing events of sufficient contrast and coherence are seen, one can estimate the rotation period. However, for a star like the Sun this is not always possible because the short lifetime of active regions (Solanki 2003) compared to the mean solar rotation period leads to an incoherent signature in integrated light. 
Asteroseismology is a tool that can be used to independently measure stellar rotation. In stars like the Sun the outer convective zone randomly excites acoustic oscillations (called $p$-modes) that propagate through the stellar interior. These oscillation modes can be described by a set of spherical harmonic functions of angular degree $l$ and azimuthal order $m$, as well as radial order $n$. Modes with $|m|>0$ travel around the rotation axis of the star in prograde and retrograde motion. For a non-rotating star the frequencies of these modes are degenerate with that of the $m=0$ modes, but become Doppler shifted if the star is rotating. This frequency shift, or splitting, is linearly related to the rotation rate of the star (see, e.g., Aerts et al. 2010, for details). This effect has been exploited to image the internal rotation in the Sun (see, e.g., Schou et al. 1998).

Using the high-quality observations of stellar light curves from space borne missions such as CoRoT (Fridlund et al. 2006) and Kepler (Borucki et al. 2010), it is possible to detect this frequency shift of the azimuthal modes in stars. This was done for a sample of subgiant stars by Deheuvels et al. $(2012,2014)$, for which it is possible to measure radial differential rotation because of the presence of mixed modes. These modes are sensitive to conditions in both the core and the outer envelope, thereby revealing the rotation rate at different depths in the star. This has been achieved for a wide variety of stars such as pulsating B-type stars (e.g., Aerts et al. 2003, Pamyatnykh et al. 2004), white dwarfs (Charpinet et al. 2009), and a main-sequence A-star (Kurtz et al. 2014). However, stars like the Sun only exhibit pure acoustic modes, which are primarily sensitive to conditions in the outer envelope, and so measurements are dominated by the rotation rates in this part of the star. The average rotation for a few Sun-like stars has been measured using data from CoRoT (Gizon et al. 2013) and Kepler (Van Eylen et al. 2014, Lund et al. 2014b). In this paper we perform an asteroseismic analysis of six Sun-like main-sequence stars observed by Kepler, and measure the rotational splittings from their oscillation spectra.

\subsection{Analysis}

The splitting of oscillation modes by rotation in Sun-like stars is typically only seen as a broadening rather than a distinct separation of the modes, caused by the combined effect of the mode linewidths and the slow rotation rates. We handpicked Sun-like stars with the longest observed time series to get the highest possible frequency resolution, and a high signal-to-noise ratio near the $p$-mode envelope. The typical length of a time series used in this work spans $\sim 3$ years. We defined these stars as Sun-like based on their reported temperatures $T_{\text {eff }} \sim 6000 \mathrm{~K}$ and surface gravities $\log g \gtrsim 4$ from spectroscopic measurements by Bruntt et al. (2012) and Molenda-Żakowicz et al. (2013). We found six stars with these characteristics that also have visible rotational splitting of the $l=1$ and $l=2$ modes.

We used the Lomb-Scargle method as applied by Frandsen et al. (1995) to compute the power spectrum of each light curve. We fit a model of the oscillation modes to small segments of the power spectrum spanning a set of $l=2,0,1$. These mode sets are all consecutive in frequency and together span the $p$-mode envelope of the star in question (see Table 3.1). We used maximum likelihood estimation to find the best-fit solution and obtain a rotational splitting for each mode set. 


\subsubsection{Observations}

We used short-cadence ( $\sim 58$ second) white light observations from the NASA Kepler mission from March 2009 until the end of the mission in early 2013. The data were obtained from the Mikulski Archive for Space Telescopes ${ }^{1}$. We used data that was preprocessed by the PDC_MAP and msMAP pipelines (Smith et al. 2012, Thompson et al. 2013) prior to release. In some cases we found narrow peaks caused by residual instrumental effects (Christiansen et al. 2011), although none of these overlapped with the $p$-mode frequencies. However, we note that the various background noise terms which we included in our model could potentially be influenced by the presence of instrumental peaks.

The Kepler Input Catalogue (KIC) numbers for the analyzed stars are shown in Table 3.1, along with the spectroscopic effective surface temperature and surface gravity measurements (Bruntt et al. 2012, Molenda-Żakowicz et al. 2013).

\subsubsection{Power spectrum model}

We fit the power spectrum with a model consisting of a constant noise level, two frequencydependent Harvey-like noise terms (see Equation 1 in Aigrain et al. 2004), in addition to the individual oscillation modes. We model these as a sum of Lorentzian profiles as per Equation 10 in Handberg and Campante (2011), each consisting of mode power, frequency, and linewidth.

We perform an initial fit of the background noise components to the entire spectrum of each star. These background terms are caused by various processes in the stellar photosphere such as granulation and magnetic activity, and span a wide range in frequency that often overlaps with the $p$-mode oscillations. We found that using two background terms was sufficient to account for the noise down to $\sim 10-100 \mu \mathrm{Hz}$, while the $p$-mode oscillations of the stars considered here have frequencies $>1000 \mu \mathrm{Hz}$. The fit parameters describing the background are subsequently kept fixed when fitting the $p$-mode oscillations.

We divide the p-mode envelope into segments of length roughly equal to the separation between radial orders (called the large frequency separation), and centered approximately between the $l=0$ and $l=1$ modes. Thus a segment contains a set of modes of angular degree $l=2,0$, and 1 (see Fig. 3.1), which we fit separately from the other sets in the spectrum.

For each angular degree $l$ there is multiplet of $2 l+1$ azimuthal modes, where, for a slowly rotating star, the components are mutually separated by an amount proportional to the stellar rotation rate $\Omega$. The frequencies of these modes can be expressed as

$$
v_{n l m}=v_{n l}+m \frac{\Omega}{2 \pi}\left(1-C_{n l}\right) \approx v_{n l}+m \delta v,
$$

where $v_{n l}$ is the frequency of the central $m=0$ mode, with the $|m|>0$ modes displaced from this frequency by the effect of rotation. The value $C_{n l}$ is small for modes of $n \gtrsim 20$ in Sun-like stars and is considered negligible. We can therefore approximate the frequency

\footnotetext{
${ }^{1}$ http://archive.stsci.edu/kepler/
} 


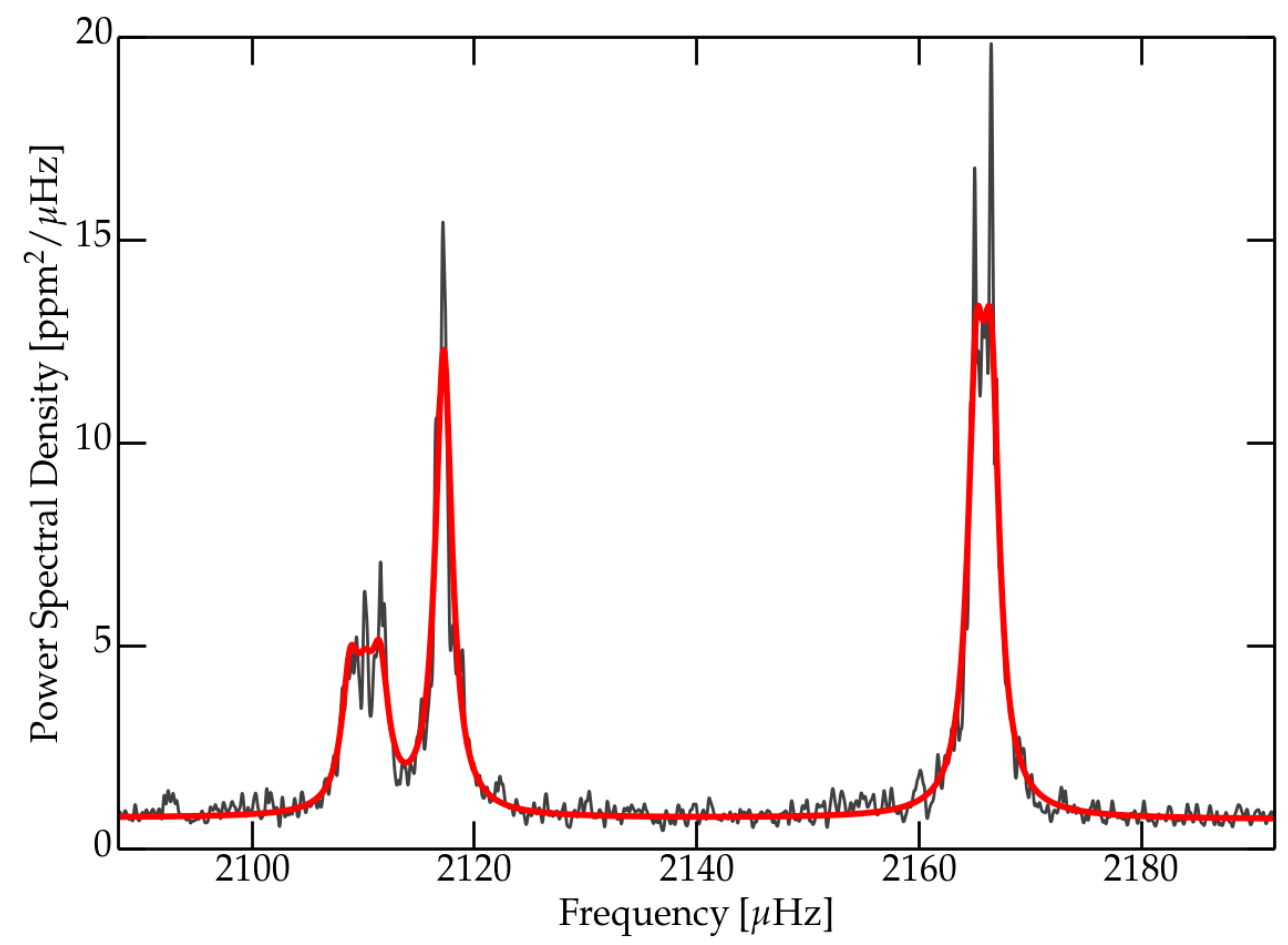

Figure 3.1: Example of a local fit performed to a segment (6th modeset) of the power spectrum of KIC006106415. The power spectrum smoothed with $0.1 \mu \mathrm{Hz}$ wide Gaussian kernel is shown in black. The red curve shows the best-fit model.

shift of the azimuthal orders by the amount $m \delta v$, where the rotational splitting $\delta v$ is equivalent to the rotation frequency of the star. We assume a common rotational splitting for the modes of a given mode set.

The amplitude of the Lorentzian profiles is a product of the mode height and the mode visibility. The mode heights are left as free parameters, and are assumed equal for all the components of a given $l$. The mode visibility is a function of the inclination $i$ of the star, where we fit a common inclination for the modes of each set. We use the form of the mode visibility as in Gizon and Solanki (2003).

For stars that rotate pole-on relative to our point of view $(i=0)$, the visibility of the $|m|>0$ modes approach zero, and so rotation cannot be measured. However, if a splitting of the $l=1$ or $l=2$ modes can be measured the different visibilities of the modes allow us to infer the inclination of the stellar rotation axis, which is not easily done using other types of observations like spectroscopy, for example.

Each mode has a finite width proportional to the lifetime of the oscillations, which is typically only on the order of a few days for Sun-like stars. For slowly rotating stars the rotational splitting may be small compared to the broadening caused by the lifetime of the mode. This makes it difficult to identify the individual azimuthal orders. However, the $l=0$ mode is unaffected by rotation so the linewidth of this mode can be taken as representative of the $l=1$ and $l=2$ modes and their associated azimuthal components (Chaplin et al. 1998). Thus, for each set of $l=2,0,1$ modes we assume a common mode linewidth. 


\subsubsection{Fitting}

We used a Markov chain Monte Carlo (MCMC) sampler $^{2}$ (Foreman-Mackey et al. 2013) to find the best-fit solution. The likelihood was computed using a $\chi^{2}$ probability density function as in Anderson et al. (1990). We used the MCMC chains to compute the marginalized posterior distributions for each parameter, where we adopt the median of each distribution as a robust measure of the best-fit parameter value. We estimated the lower and upper errors for each parameter by the 16th and 84th percentile values of the posterior distributions. Figure 3.1 shows a model fit to a section of the power spectrum of KIC006106415.

For the mode heights, central frequencies and width the initial positions of the walkers were randomly chosen from a normal distribution centered on a manually-determined initial guess. Each distribution had a standard deviation equal to $10 \%$ of the initial guess value in order to provide the walkers with sufficient initial coverage of parameter space. For the inclination and splitting parameters we opted to use a uniform random distribution between $0-90^{\circ}$ and $0-2 \mu \mathrm{Hz}$, respectively, since these two parameters are known to be non-linearly correlated.

We used a probability distribution function of $\sin i$ on the inclination angle as a prior. We used uniform priors for all other parameters. These were only constrained for the $l=2$ and $l=0$ frequencies and the rotational splitting, such that the frequencies of each mode could not overlap. Initial testing showed that the walkers of the MCMC chain would sometimes switch the frequencies of these two modes because of their proximity. We found that this limitation on $\delta v$ did not bias the measurements or errors after inspection of the posterior distributions.

We used 100 walkers to generate the MCMC chains which were allowed to run for 1200 steps, giving us 120000 samples in the available parameter space. Although not strictly necessary owing to the rapid mixing of the walkers, we chose to disregard the first 600 steps as the burn-in phase of the MCMC chains.

\subsection{Rotation and inclination as a function of frequency}

The complete list of fit values and associated errors (Table 2) is available as online material via the CDS. The fit values for the rotational splitting $\delta v$ and the inclination angle $i$ are presented in Fig. 3.2. We compute a variance weighted mean of the splittings measured for each star, and list these in Table 3.1. The posterior distributions of the rotational splittings are approximately Gaussian around the mean (see Fig. 3.3), so the variance is representative of the errors associated with each splitting. This is not true for the posterior distributions of the inclinations and so we cannot apply this to obtain a weighted mean value representative of the inclination of each star. We therefore only list an unweighted mean of the inclination measurements with typical errors of $\sim 20^{\circ}$.

A few stars (e.g., KIC006106415) appear to show a marginal trend in the splittings with increasing frequency. To test this further we computed a $\chi^{2}$ and the associated $\mathrm{p}$ values based on the variance weighted mean splitting, i.e., a constant rotational splitting with frequency. We found that the $\chi^{2}$ values ranged between $0.6-3.9$ and the p-values

\footnotetext{
${ }^{2}$ The affine invariant sampler in the EMCEE package for Python, http://dan.iel.fm/emcee/current/
} 


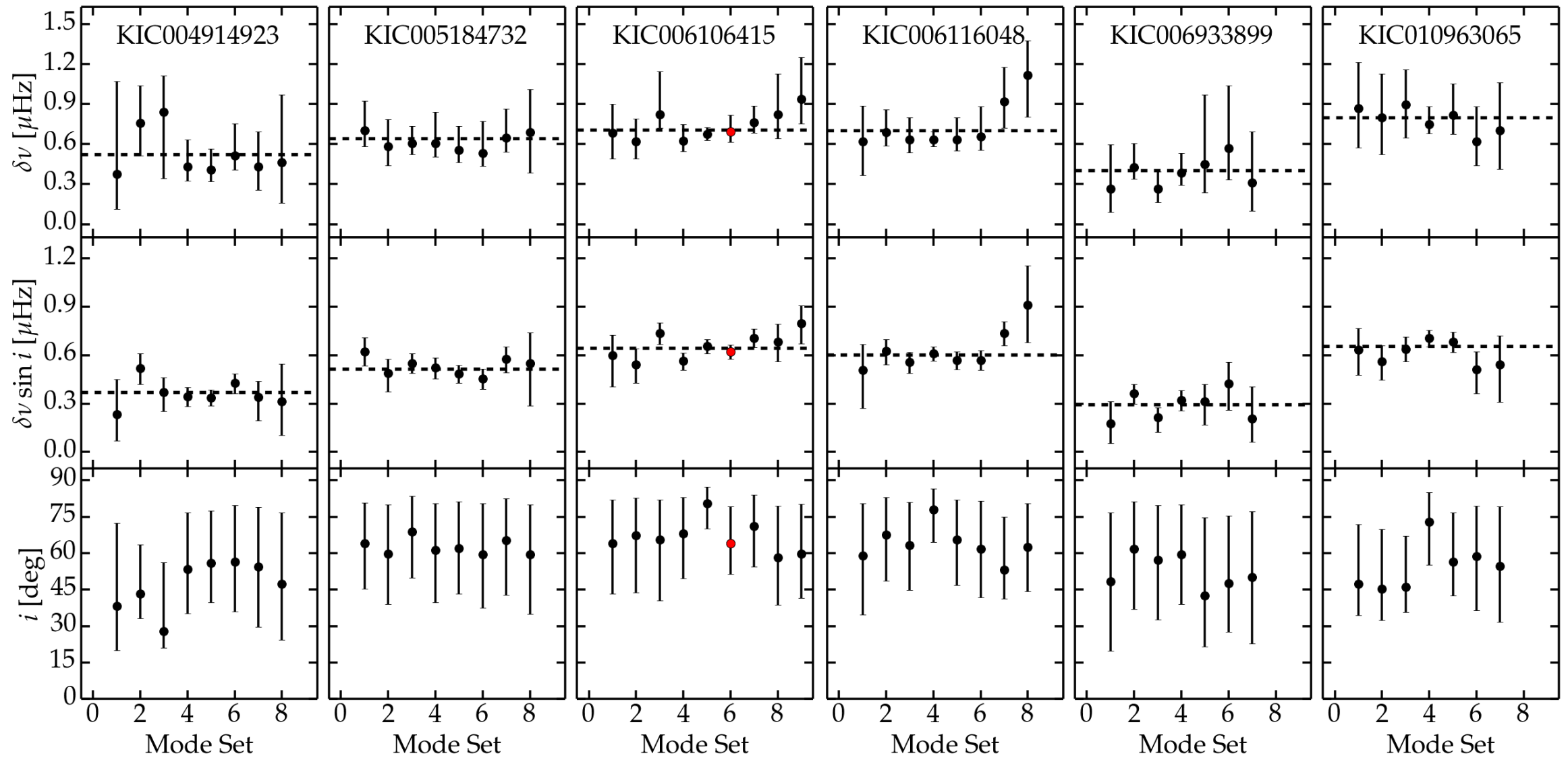

Figure 3.2: The measured splitting $\delta v$ and inclination $i$ of each mode set. The points show the results of the local fit as a function of the mode sets in each power spectrum. The error bars denote the 16 th and $84 t h$ percentile values of the marginalized posterior distributions obtained from the MCMC samples. Dashed lines indicate the variance weighted mean of the values, using the variance of each posterior distribution. Red points show the mode set used in Fig. 3.1. 
Table 3.1: Variance weighted mean rotational splittings $\langle\delta v\rangle$, inclination $\langle i\rangle$, and rotation period $\Omega / \Omega_{\odot}$ for the six Sun-like stars. The effective temperature $T_{\text {eff }}$, surface gravity $\log g$, and frequency intervals considered for each star are also listed, where each interval is divided into segments of length approximately equal to the large frequency separation. The variance weighted mean splittings $\langle\delta v\rangle$ are shown as dashed lines in Fig. 3.2, where the listed errors are the standard deviations of the weighted mean values. We note that the posterior distributions for the $\delta v$ are only approximately Gaussian. The posterior distributions of inclination measurements cannot be approximated as a Gaussian and so we only show the unweighted mean of the inclinations where typical errors are $\sim 20^{\circ}$. The reader should not use the mean values and associated errors reported here, but should refer to the online material for more accurate values for each mode set. For comparison, the final column shows the stellar rotation rate relative to the solar value (we used $\Omega_{\odot}=0.424 \mu \mathrm{Hz}$ ).

\begin{tabular}{llcccccc}
\hline Star & $T_{\text {eff }}[\mathrm{K}]$ & $\log g\left[\mathrm{~cm} / \mathrm{s}^{2}\right]$ & Fit interval $[\mu \mathrm{Hz}]$ & $\langle\delta v\rangle[\mu \mathrm{Hz}]$ & $\langle\delta v \sin i\rangle[\mu \mathrm{Hz}]$ & $\langle i\rangle[\mathrm{deg}]$ & $\Omega / \Omega_{\odot}$ \\
\hline KIC004914923 & $5808 \pm 92$ & $4.28 \pm 0.21$ & $1429-2135$ & $0.522 \pm 0.074$ & $0.371 \pm 0.029$ & 54 & $1.23 \pm 0.29$ \\
KIC005184732 & $5669 \pm 97$ & $4.07 \pm 0.21$ & $1632-2400$ & $0.643 \pm 0.063$ & $0.517 \pm 0.027$ & 62 & $1.52 \pm 0.12$ \\
KIC006106415 & $6050 \pm 70$ & $4.40 \pm 0.08$ & $1677-2609$ & $0.708 \pm 0.038$ & $0.647 \pm 0.022$ & 64 & $1.67 \pm 0.27$ \\
KIC006116048 & $5991 \pm 124$ & $4.09 \pm 0.21$ & $1620-2425$ & $0.703 \pm 0.053$ & $0.603 \pm 0.024$ & 69 & $1.66 \pm 0.36$ \\
KIC006933899 & $5837 \pm 97$ & $4.21 \pm 0.22$ & $1157-1662$ & $0.404 \pm 0.078$ & $0.296 \pm 0.034$ & 57 & $0.95 \pm 0.27$ \\
KIC010963065 & $6097 \pm 130$ & $4.00 \pm 0.21$ & $1760-2475$ & $0.801 \pm 0.079$ & $0.656 \pm 0.032$ & 56 & $1.89 \pm 0.20$
\end{tabular}


between $0.69-0.998$, indicating that the measurements are consistent with a constant splitting over frequency. We noted that the errors on the rotational splittings are likely to be anti-correlated with the errors on the inclinations (see Fig. 3.3). We therefore also computed posterior distributions of $\delta v \sin i$ (middle row in Fig. 3.2), and performed the same test for constant rotation. The computed $\chi^{2}$ and p-values were between $3.3-7.7$ and $0.36-0.77$, respectively, i.e., the variations seen in $\delta v \sin i$ are still consistent with uniform rotation in these stars. We therefore find no evidence of differential rotation in these stars. In Sun-like stars the mode linewidths increase strongly with frequency (Chaplin et al. 1998). This means that using a common linewidth likely ceases to be a good approximation for the last few mode sets at higher frequencies, thus introducing a bias in the splitting parameter.

The inclination of the rotation axis is an important parameter for characterizing exoplanetary systems and constraining models of planet formation and evolution (e.g., Nagasawa et al. 2008). However, we found that the inclination angles are very poorly constrained when using a single mode set, even with these prime examples from the Kepler database. In Fig. 3.3 we show the marginalized posterior distributions for the fit shown in Fig. 3.1. The posterior distribution reveals that the inclination angle is dominated by the $\sin i$ prior, i.e., an individual mode set yields very little information about the stellar inclination axis. In this case, based on the posterior distribution we could only conclude that $i \lesssim 45^{\circ}$ is unlikely. This is a common trait of the posterior distributions for the other stars in our sample, and some are even less constrained so that we can only rule out $i \lesssim 20^{\circ}$. The relatively high inclination angles that we measure are expected when considering these stars were chosen by eye to have a visible splitting, or at least a broadening of the $l=2$ and $l=1$ modes. This selection naturally biases the sample of stars toward highly inclined configurations (see Fig. 2. in Gizon and Solanki 2003).

These stars were specifically selected for this study since they have visible rotational splittings. When using high signal-to-noise observations such as these, it is a simple matter of fitting just the central mode sets of the $p$-mode envelope in order to obtain a reliable measure of the rotational splitting. Furthermore, these high-quality data offer the tantalizing possibility of measuring radial differential rotation. From our measurements we have determined that these Sun-like stars are unlikely to have variations in rotational splittings larger than $\sim 40 \%$. Improvements to the fitting method, e.g., linewidth parametrization or a global fit to the power spectrum, could reduce the uncertainties on the splitting measurements and potentially reveal the signatures of differential rotation. 


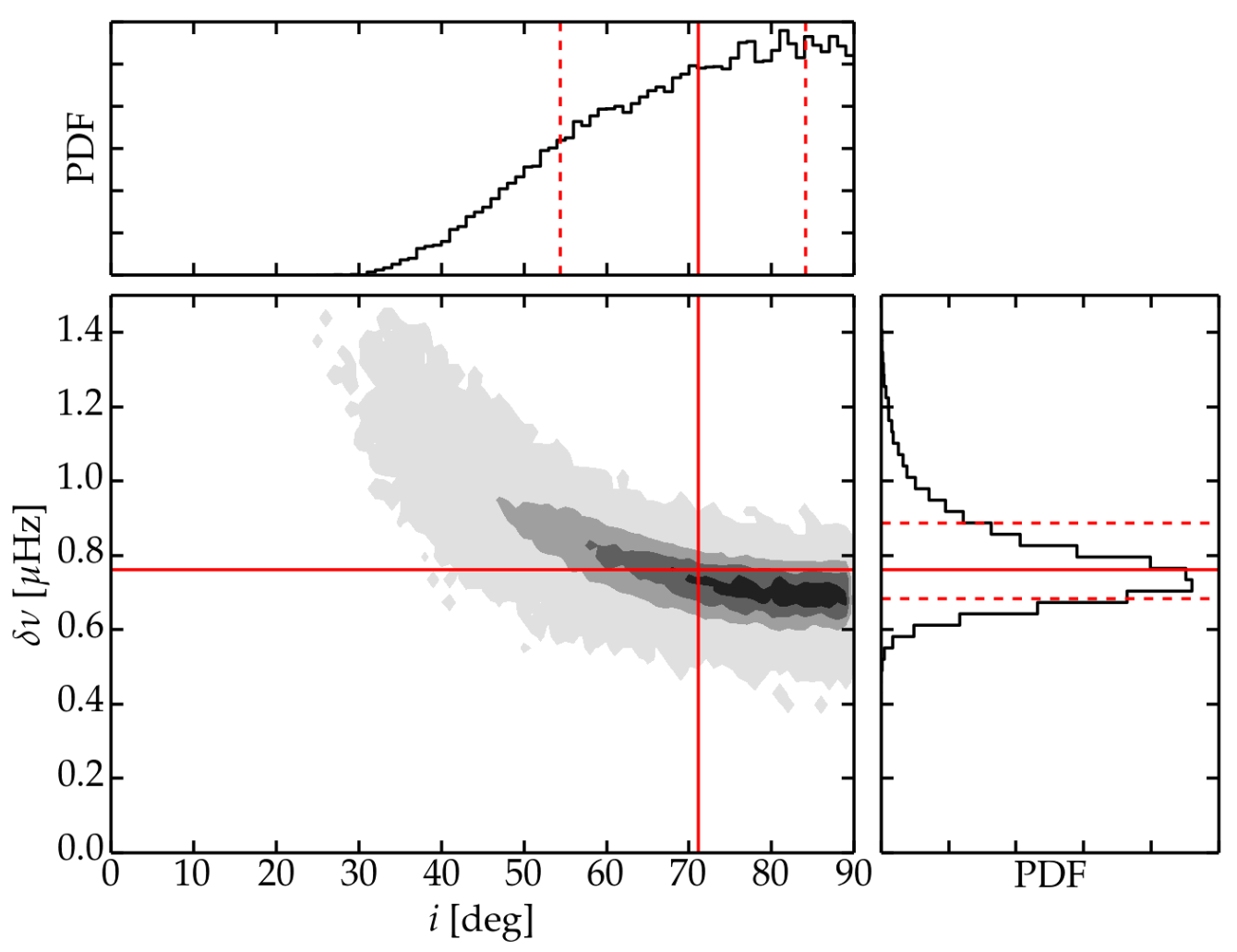

Figure 3.3: Bottom left: A 2D representation of the marginalized posterior distributions for the rotational splitting $\delta v$ and the inclination of the rotation axis $i$. Top and right frames show the projection onto each axis in solid black. The solid red lines indicate the median along each axis, and dashed red lines are 16th, 84th percentile values. These distributions are obtained from the local fit to the modes shown in Fig. 3.1. 



\section{Paper III - Constraining differential rotation of Sun-like stars from asteroseismic and starspot rotation periods}

This chapter has been published in 2015 in A\&A vol. 582, page A10. The work was carried out and written by myself, under the supervision of L. Gizon, H. Schunker and J. Schou from the Max Planck Institute for Solar System Research.

\subsection{Summary of Paper III}

In previous work, we identified six Sun-like stars observed by Kepler with exceptionally clear asteroseismic signatures of rotation. Here, we show that five of these stars exhibit surface variability suitable for measuring rotation. We compare the rotation periods obtained from light-curve variability with those from asteroseismology in order to further constrain differential rotation. The two rotation measurement methods are found to agree within uncertainties, suggesting that radial differential rotation is weak, as is the case for the Sun. Furthermore, we find significant discrepancies between ages from asteroseismology and from three different gyrochronology relations, implying that stellar age estimation is problematic even for Sun-like stars.

\subsection{Introduction}

Recently, asteroseismology has become a valuable tool to study the internal rotation of stars. This has been done on a variety of different stars (see, e.g., Aerts et al. 2003, Charpinet et al. 2009, Kurtz et al. 2014), including Sun-like stars (Gizon et al. 2013, Davies et al. 2015). Asteroseismic measurements of rotation in Sun-like stars have generally been limited to the average internal rotation period. More recently, Nielsen et al. (2014) identified six Sun-like stars where it was possible to measure rotation for independent sets of oscillations modes and found that radial differential rotation is likely to be small.

From the Sun, we know that different methods for measuring rotation, e.g., spot tracing (D'Silva and Howard 1994) and helioseismology (Schou et al. 1998), show the outer envelope of the Sun rotating differentially. The solar surface rotation period changes by 
4 Paper III - Constraining differential rotation of Sun-like stars from asteroseismic and starspot rotation periods

approximately 2.2 days between the equator and $40^{\circ}$ latitude (Snodgrass and Ulrich 1990), while in the radial direction the rotation period changes by $\sim 1$ day in the outer few percent of the solar radius (Beck 2000). While small on the Sun, differential rotation could be larger on other stars. With data available from the Kepler mission (Borucki et al. 2010), it is now possible to compare the measurements of rotation using both asteroseismology and observations of surface features.

Differences between the results of the two methods would imply that differential rotation exists between the regions where the two methods are sensitive; namely, the nearsurface layers for asteroseismology, and the currently unknown anchoring depth of active regions in Sun-like stars. However, given the similar scales of the radial and latitudinal differential rotation seen in the Sun, both of these mechanisms may contribute to an observed difference. On the other hand, agreement between the two methods would suggest weak differential rotation. Furthermore, this would also mean that we can measure rotation with asteroseismology in inactive stars that exhibit few or no surface features at all, thus giving us an additional means of calibrating gyrochronology relations (e.g., Skumanich 1972, Barnes 2007, Barnes and Kim 2010), which so far have primarily relied on measuring the surface variability of active stars.

However, the acoustic modes (or $p$-modes) used to measure rotation in Sun-like stars are damped by surface magnetic activity (Chaplin et al. 2011). It is therefore difficult to find stars with both a high signal-to-noise oscillation spectrum and coherent signatures of rotation from surface features. Nielsen et al. (2014) identified six Sun-like stars from the Kepler catalog with exceptional signal to noise around the p-mode envelope. Five of these stars exhibit rotational variability from surface features. We compare the surface variability periods with those measured from asteroseismology, with the aim of further constraining the near-surface differential rotation of these stars.

\subsection{Measuring rotation periods}

We use the $\sim 58$ second cadence observations from the Kepler satellite to capture the high frequency acoustic oscillations, and the $\sim 29$ minute cadence observations to search for surface variability. The stars we identified were chosen because of their similarity to the Sun with respect to surface gravity and effective temperature, and for having a high signal-to-noise ratio at the p-mode envelope. We use all the available data ${ }^{1}$ for each star, from quarter 1 to 17, spanning approximately four years.

\subsubsection{Asteroseismic rotation periods}

The oscillation modes in a star can be described with spherical harmonic functions, characterized by the angular degree $l$ and azimuthal order $m$, and a radial component with order $n$. When the star rotates the modes with azimuthal order $m>0$ become Doppler shifted, and if this can be measured, the rotation of the star can be inferred. For Sun-like stars, the oscillation modes have a sensitivity weighted toward the surface of the star, and so the asteroseismic measurements predominantly probe the rotation of the star in this region (see, e.g., Lund et al. 2014a).

\footnotetext{
${ }^{1}$ Downloaded from the Mikulski Archive for Space Telescopes, http://archive.stsci.edu/kepler/
} 


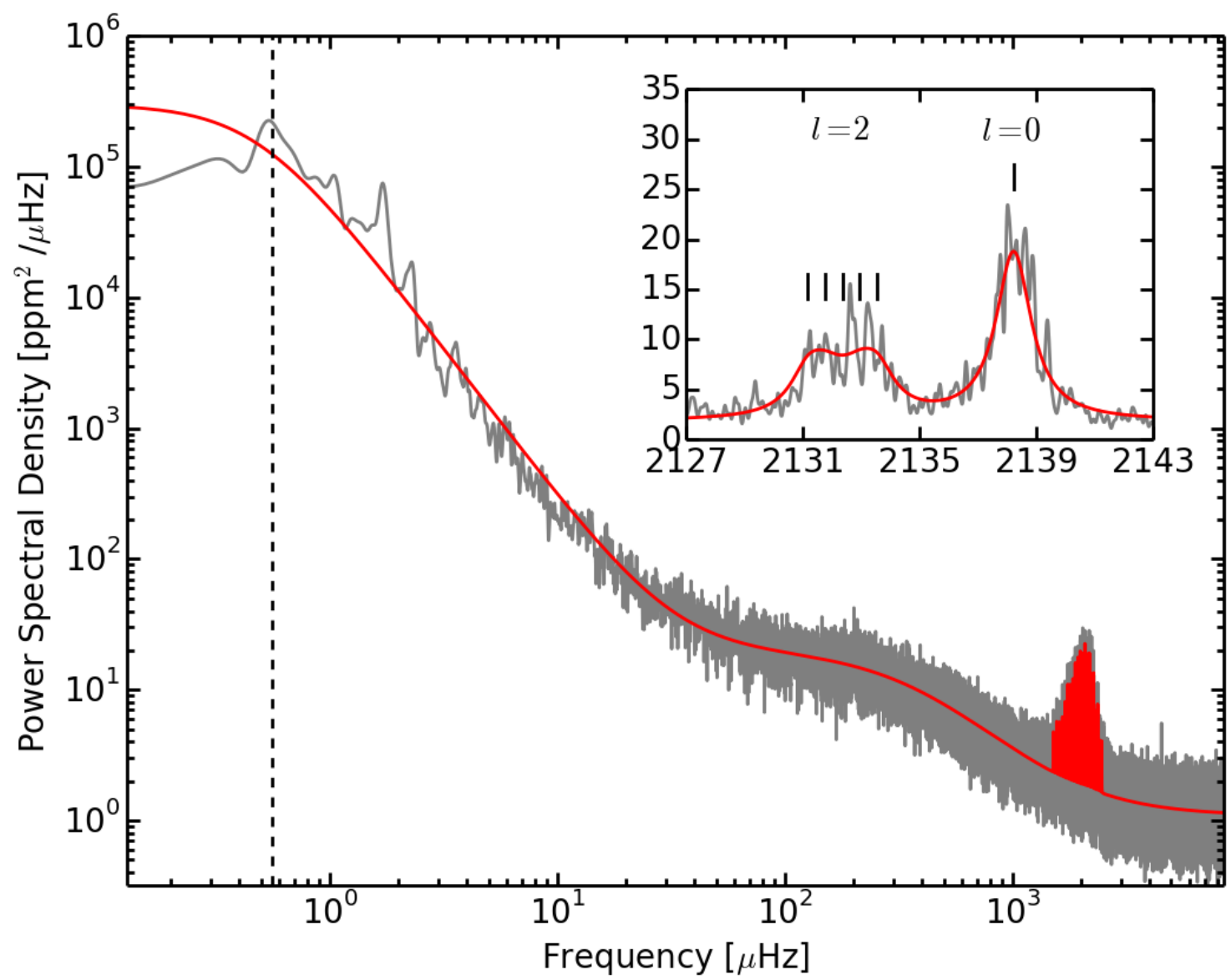

Figure 4.1: Power density spectrum of the short cadence white light photometry time series of KIC005184732. Gray represents the spectrum smoothed with a $0.1 \mu \mathrm{Hz}$ wide Gaussian kernel, with the best-fit model shown in red. Black dashed indicates the surface variability frequency. The inset shows a zoom of the p-mode envelope at an $l=2,0$ pair, where the black markers indicate the individual $m$-components. The separation between components of the $l=2$ mode is the rotational splitting.

To measure this frequency splitting we fit a model to the power spectral density. The modes in a solar-like oscillator are stochastically excited and damped oscillations can be described by a series of Lorentzian profiles,

$$
P(v)=\sum_{n} \sum_{l=0}^{3} \sum_{m=-l}^{l} \frac{\mathscr{E}_{l m}(i) S_{n l}}{1+\left(2 / \Gamma_{n l m}\right)^{2}\left(v-v_{n l m}\right)^{2}}+B(v),
$$

where $v$ is the frequency, and $B(v)$ is the background noise. Here the sums are over the radial orders (typically $\sim 8$ values of $n$ ), the angular degrees $l \leq 3$ and the azimuthal orders $-l \leq m \leq l$. Not all radial orders show clear $l=3$ modes, and so only a few $l=3$ modes are included in the fits, depending on the star. The mode power in a multiplet, $S_{n l}$, is a free parameter. The mode visibility $\mathscr{E}_{l m}(i)$ is a function of the inclination angle $i$ of the rotation axis relative to the line of sight (see Gizon and Solanki 2003). We assume here that all modes share the same value of $i$.

The frequencies $v_{n l m}$ of the modes reveal the rotation information. For Sun-like stars 
4 Paper III - Constraining differential rotation of Sun-like stars from asteroseismic and starspot rotation periods

rotating no more than a few times the solar rotation rate the mode frequencies can be parametrized as $v_{n l m} \approx v_{n l}+m \delta v$, where $\delta v$ is the rotational splitting. In our model fit we assume that the stellar interior rotates as a solid body, so that all modes share a common rotational splitting.

The full widths $\Gamma_{n l m}=\Gamma\left(v_{n l m}\right)$ of the Lorentzian profiles depend on the mode lifetimes, which in turn depend on the mode frequencies $v_{n l m}$. In the Sun this variation with frequency can be modeled by a low-order polynomial (Stahn 2010). We therefore opt to parametrize the mode widths as a third-order polynomial in $\left(v-v_{\max }\right)$, where $v_{\max }$ is the frequency at maximum power of the p-mode envelope. Note that only the $l=0$ modes contribute to the fit of this function since they are not broadened or split by rotation.

The background noise level $B(v)$ is caused by several terms: the very long-term variability (hours to days) from magnetic activity, the variability (tens of minutes) from stellar surface granulation, and the photon noise. For asteroseismology applications, these noise components can be adequately described by two Harvey-like background terms (e.g., Handberg and Campante 2011) and a constant term to account for the white photon noise.

We find the best fit with maximum likelihood estimation using a Markov Chain Monte Carlo sampler (Foreman-Mackey et al. 2013). The best-fit values of each parameter are estimated by the median of the corresponding marginalized posterior distribution, and the 16th and 84th percentile values of the distributions represent the errors. We fit the entire spectrum, including all mode parameters and background terms simultaneously (a so-called global fit), in contrast to Nielsen et al. (2014) who fit the background and separate sets of p-modes individually. A comparison between the global rotational splitting obtained in this work, and the mean of the rotational splittings measured by Nielsen et al. (2014) are shown in Table 4.1.

An example spectrum of KIC005184732 is shown in Fig. 4.1. The background terms dominate the low frequency end of the spectrum, while the p-mode envelope appears clearly above the noise level at $\sim 1800 \mu \mathrm{Hz}$. The inset shows a part of the p-mode envelope, where the splitting of an $l=2$ mode is clearly visible.

\subsubsection{Surface variability periods}

The rotation of stars can also be inferred from the periodic variability of their light curves caused by surface features on the stellar disk, such as active regions. For the Sun, active regions are good tracers of the surface rotation of the plasma, to within a few percent (Beck 2000). In other stars this difference could potentially be larger.

From the periodic variation of the stellar light curve it is relatively straightforward to identify the rotation period of the star, and this has been done using automated routines for tens of thousands of stars (Nielsen et al. 2013, Reinhold and Reiners 2013, McQuillan et al. 2014). However, these routines typically rely on coherent surface variability over long time periods. The stars studied here do not show simple and regular surface variability and, therefore, they do not appear in these catalogs. Furthermore, the PDC_MAP pipeline (Smith et al. 2012, Stumpe et al. 2014) is known to suppress variability on timescales longer than 20 days (Christiansen et al. 2013)

We therefore manually reduce the raw pixel data for each star, and search this and the PDC_MAP reduced data for signs of rotational variability. To reduce the raw data, we 

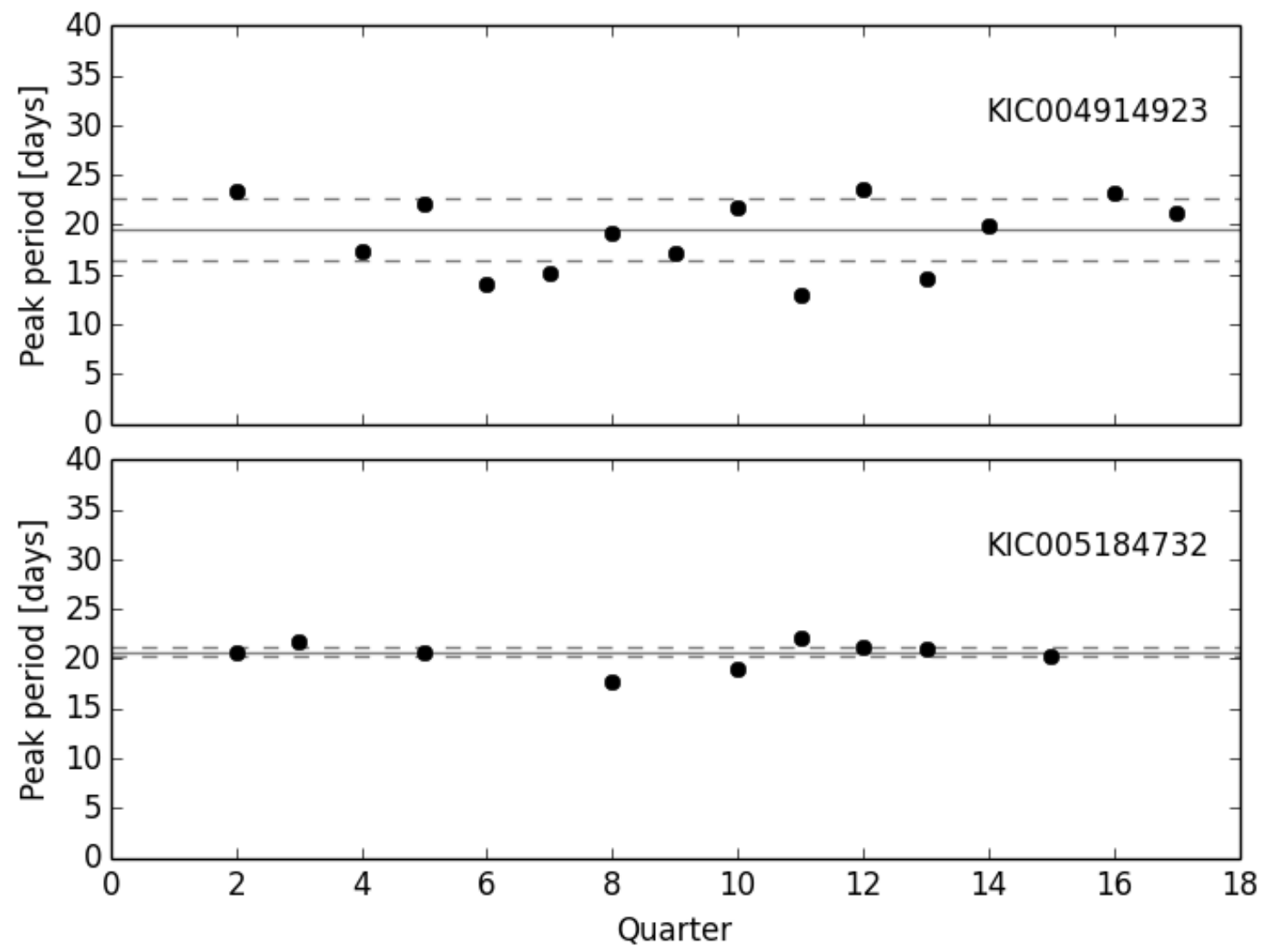

Figure 4.2: Peak periods in the Lomb-Scargle periodogram as a function of observation quarter for KIC004914923 (top) and KIC005184732 (bottom). Solid gray shows the median period, and dashed gray shows the median absolute deviation.

use the kepcotrend procedure in the PyKe software package ${ }^{2}$. The PyKe software uses a series of so-called cotrending basis vectors (CBVs) in an attempt to remove instrumental variability from the raw light curves. The CBVs are computed based on variability common to a large sample of stars on the detector, and so in principle represent the systematic variability. The number of CBVs to use in the reduction is not clear and we therefore compute several sets of reduced light curves using a sequentially increasing number of CBVs (up to six) for each star. We require that the variability appears in light curves reduced with different numbers of CBVs. This minimizes the risk of variability signatures that are caused by the reduction.

To measure the rotation period of each star we used the method of Nielsen et al. (2013). We compute the Lomb-Scargle periodogram (Lomb 1976, Scargle 1982) of each quarter and identify the peak of maximum power for periods less than 45 days. The median period of these peaks is used as a first order estimate of the rotation period. We then assume that active regions appear at similar latitudes, and so should have similar periods. This is done by requiring that peaks must lie within 4 median absolute deviations (MAD) from the median. The median and MAD of the remaining peak periods are the measured rotation period and error on the rotation period. Examples are shown in Fig. 4.2.

\footnotetext{
${ }^{2} \mathrm{http} / / /$ keplerscience.arc.nasa.gov/PyKE.shtml
} 
4 Paper III - Constraining differential rotation of Sun-like stars from asteroseismic and starspot rotation periods

The rotation periods of these stars measured using this method agree with those found by García et al. (2014) do Nascimento et al. (2014).

\subsection{Comparing asteroseismic and surface variability pe- riods}

In Table 4.1 we list the periods for each analysis. These values are also shown in Fig. 4.3. The asteroseismic and surface variability periods agree very well for all the stars analyzed here. However, the asteroseismic rotation period measurements are systematically lower than those from surface variability. As a first order significance estimate of this difference we fit a linear function $P_{\mathrm{S}}=a P_{\mathrm{A}}$ to the measured values. We find the best fit by maximum likelihood estimation, using the marginalized posterior distributions for the asteroseismic measurements, and assume Gaussian posterior distributions for the surface variability periods. The best-fit solution returns $a=1.044_{-0.058}^{+0.068}$, showing that the difference between the two measurement methods is insignificant (see Fig. 4.3). As a consistency check we perform an identical fit to the average rotation periods $P_{\mathrm{N}}$ from Nielsen et al. (2014), assuming Gaussian errors (see Table 4.1). We find a slope of $1.006_{-0.060}^{+0.066}$, in good agreement with the slope of $P_{\mathrm{A}}$. However, this does not account for the non-Gaussian form of the marginalized posteriors of the fits performed in that work.

Here we estimate the stellar ages from three different gyrochronology relations provided by Mamajek and Hillenbrand (2008), Barnes and Kim (2010), and García et al. (2014). These relations represent distinct methods of estimating gyrochronology ages. In all these relations, we use the asteroseismic rotation periods from Sect. 4.3.1.

The type of gyro-relation used by Mamajek and Hillenbrand (2008), first proposed by Barnes (2007), assumes that the rotation period of a star varies as powers of age and mass, where the $B-V$ color is used as a proxy for the latter. This relation is calibrated to young cluster stars with ages $\lesssim 625 \mathrm{Gyr}$, with only the Sun representing the older stellar populations. For the stars studied here we use $B-V$ values from Høg et al. (2000), using extinction values from the KIC catalog (Brown et al. 2011).

The relation by García et al. (2014) is calibrated to stars with asteroseismically determined ages from Mathur et al. (2012) and Metcalfe et al. (2014). However, this relation does not include a mass dependence since the calibrators are all similar in mass $\left(\sim 1 \mathrm{M}_{\odot}\right)$, and so this relation only depends on the rotation period of the star.

Lastly, the relation by Barnes and Kim (2010) incorporates the effects of a rapid spindown phase, experienced by very young stars $(\sim 100 \mathrm{Myr})$, into the prediction of the stellar age. This relation uses convective turnover time $\tau$ as a proxy for mass, and is also calibrated using young cluster stars, with the Sun as the only anchoring point for older stars.

We determine the convective turnover times from stellar models fit to the stellar oscillation frequencies (see online material in Nielsen et al. (2014)). These stellar models are produced using the Modules for Experiments in Stellar Astrophysics (MESA, Paxton et al. 2011, 2013), 


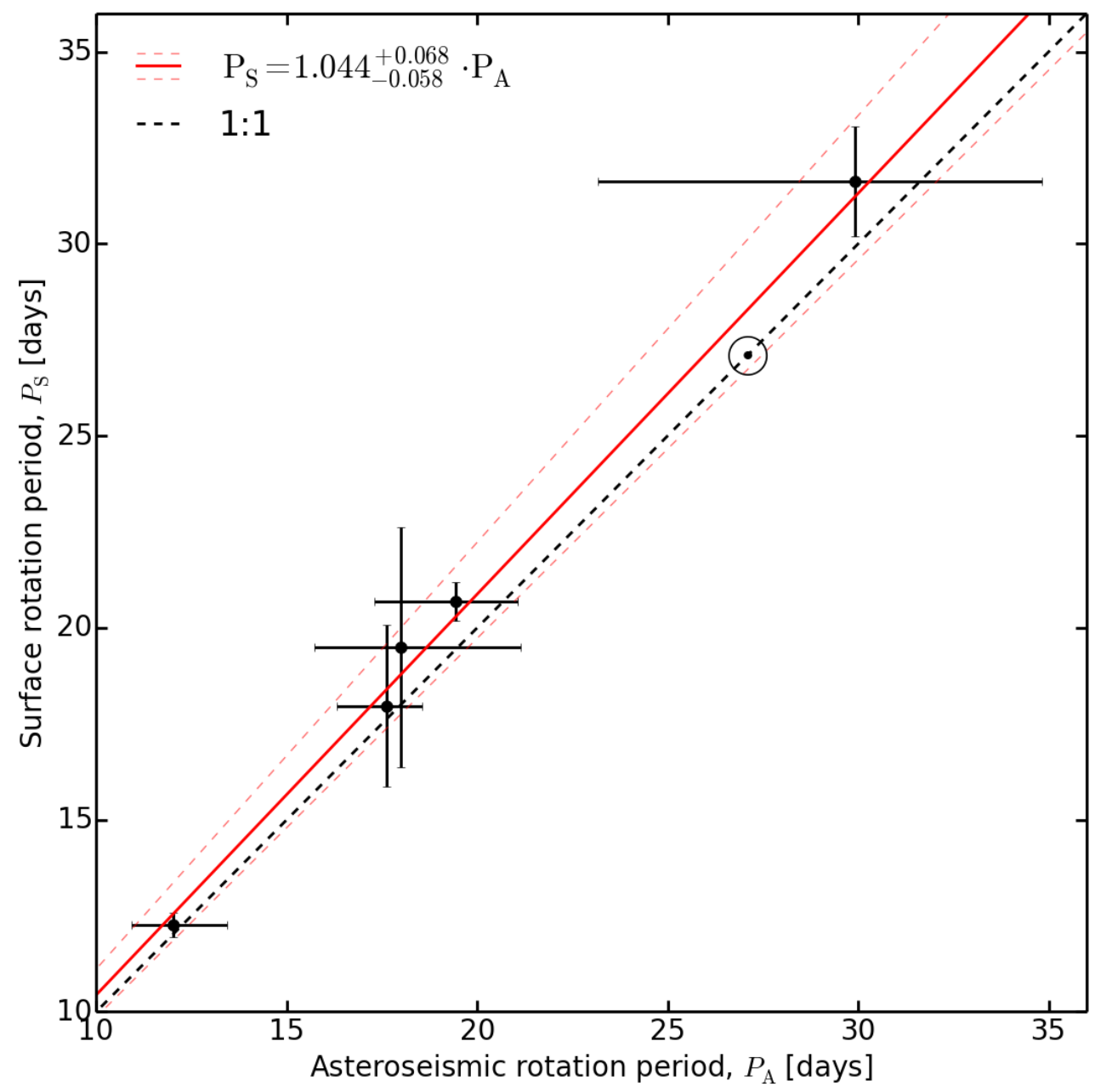

Figure 4.3: Surface variability period $P_{\mathrm{S}}$ as a function of asteroseismic period $P_{\mathrm{A}}$. The 1:1 line is shown with a black dashed line. The best-fit value and errors to the slope are shown with red solid and dashed lines, respectively. For comparison the Carrington period of 27.3 days is also shown (this is not included in the fit). 
Table 4.1: List of measured and computed parameters for the five stars. The stellar masses and radii, core hydrogen fraction $\mathrm{X}_{\mathrm{c}}$, and convective turnover time $\tau$, as well as the seismic ages are computed from the best-fit models as described in Section 4.5. The effective temperatures are adapted from Bruntt et al. (2012). The measured surface variability rotation periods $P_{\mathrm{S}}$ and asteroseismic rotation periods $P_{\mathrm{A}}$ are also listed. These periods are compared to the averaged asteroseismic rotation periods $P_{\mathrm{N}}$ from Nielsen et al. (2014). The $P_{\mathrm{N}}$ values are averages over several sets of oscillation modes. The gyrochronology ages are calculated from Barnes and Kim (2010), Mamajek and Hillenbrand (2008), and García et al. (2014).

\begin{tabular}{|c|c|c|c|c|c|}
\hline & KIC004914923 & KIC005184732 & KIC006116048 & KIC006933899 & KIC010963065 \\
\hline Mass $\left[\mathrm{M}_{\odot}\right]$ & $1.118 \pm 0.020$ & $1.205 \pm 0.025$ & $1.023 \pm 0.021$ & $1.096 \pm 0.026$ & $1.062 \pm 0.021$ \\
\hline Radius $\left[\mathrm{R}_{\odot}\right]$ & $1.378 \pm 0.009$ & $1.342 \pm 0.010$ & $1.225 \pm 0.008$ & $1.574 \pm 0.025$ & $1.220 \pm 0.009$ \\
\hline $\mathrm{X}_{\mathrm{c}}$ & 0.000 & 0.216 & 0.036 & 0.000 & 0.108 \\
\hline$\tau$ [days] & 16.94 & 23.30 & 34.34 & 28.25 & 33.03 \\
\hline $\mathrm{T}_{\mathrm{eff}}[\mathrm{K}]$ & $5880 \pm 70$ & $5865 \pm 70$ & $5990 \pm 70$ & $5870 \pm 70$ & $6090 \pm 70$ \\
\hline$P_{\mathrm{S}}[$ days $]$ & $19.49 \pm 3.12$ & $20.69 \pm 0.50$ & $17.96 \pm 2.11$ & $31.63 \pm 1.43$ & $12.27 \pm 0.32$ \\
\hline$P_{\mathrm{A}}[$ days $]$ & $17.98_{-2.27}^{+3.17}$ & $19.44_{-2.13}^{+1.63}$ & $17.61_{-1.31}^{+0.95}$ & $29.92_{-6.76}^{+4.90}$ & $12.01_{-1.09}^{+1.42}$ \\
\hline$P_{\mathrm{N}}$ [days $]$ & $22.17 \pm 3.14$ & $18.00 \pm 1.76$ & $16.34 \pm 0.89$ & $28.65 \pm 5.53$ & $14.45 \pm 1.43$ \\
\hline Seismic age [Gyr] & $6.23 \pm 0.36$ & $4.39 \pm 0.13$ & $5.70 \pm 0.21$ & $6.57 \pm 0.30$ & $4.18 \pm 0.19$ \\
\hline \multicolumn{6}{|l|}{ Gyrochronology ages [Gyr] } \\
\hline Barnes and Kim (2010) & $2.68_{-0.62}^{+0.95}$ & $2.72_{-0.54}^{+0.47}$ & $3.09_{-0.44}^{+0.36}$ & $5.58_{-1.95}^{+2.42}$ & $1.97_{-0.34}^{+0.49}$ \\
\hline Mamajek and Hillenbrand (2008) & $3.56_{-1.14}^{+1.93}$ & $2.36_{-0.53}^{+0.63}$ & $3.36_{-0.82}^{+1.14}$ & $8.57_{-3.08}^{+3.73}$ & $4.38_{-1.74}^{+3.77}$ \\
\hline García et al. (2014) & $3.25_{-0.95}^{+1.52}$ & $3.65_{-0.95}^{+1.37}$ & $3.05_{-0.67}^{+0.92}$ & $7.83_{-2.84}^{+4.78}$ & $1.49_{-0.34}^{+0.51}$ \\
\hline
\end{tabular}


with the same input physics as in Ball and Gizon (2014). Initial fits were determined using the SEEK method (Quirion et al. 2010) to compare a grid of models to observed spectroscopic and global asteroseismic parameters. The models cover the mass range $0.60-1.60 \mathrm{M}_{\odot}$ and initial metal abundances $Z$ in the range $0.001-0.040$. The helium abundance was presumed to follow the enrichment law $Y=0.245+1.45 Z$ and the mixing length parameter was fixed at $\alpha_{\odot}=1.908$.

As initial guesses, we used the median values of the grid-based fit and also generated ten random, uniformly-distributed realizations of the parameters. The 11 initial guesses were optimized in five parameters (age, mass, metallicity, helium abundance, and mixing length) using a downhill simplex (Nelder and Mead 1965) to match spectroscopic data (Bruntt et al. 2012) and individual oscillation frequencies, computed with ADIPLS (Christensen-Dalsgaard 2008) and corrected according to the cubic correction by Ball and Gizon (2014). As in Ball and Gizon (2014), the seismic and nonseismic observations are weighted only by their respective measurement uncertainties. Best-fit parameters and uncertainties are estimated from ellipses bounding surfaces of constant $\chi^{2}$ for all the models determined during the optimizations, corresponding to a total of about 3000 models for each star.

The best-fit models yield local convective velocities and we use these to compute $\tau$ as in Kim and Demarque (1996), these are listed in Table 4.1. From these values of $\tau$ and the asteroseismic rotation periods from section 4.3.1, we compute the gyrochronology ages using Eq. 32 from Barnes and Kim (2010) and the suggested calibration values therein.

\subsection{Gyrochronology}

In addition to providing values of $\tau$, the stellar models also produce estimates of the stellar ages. In Fig. 4.4 these ages are compared with those derived from the three gyrochronology relations. It is immediately obvious that the gyro-relations are in general not consistent with the seismic ages. For some stars a particular gyro-relation may agree well with the seismic age, but for other stars that same relation deviates significantly, as seen, e.g., for the ages from García et al. (2014) and Mamajek and Hillenbrand (2008) relations for KIC005184732 and KIC010963065. However, it appears that the gyro-relations tend to underestimate the stellar ages relative to the seismic values.

For the star KIC006933963, the gyro-relations predict an age older than the stellar models, which is likely because this star is a subgiant, with a slightly contracted core and expanded envelope. Thus, by angular momentum conservation one expects the surface and near-surface regions to have slowed down faster than the gyro-relations predict, leading to an overestimated age. KIC004914923 also appears to be a subgiant based on its core hydrogen content (see Table 4.1), but close inspection of the best-fit stellar model shows that this star has only just moved off the main sequence, and so may still adhere to these gyro-relations.

It should be noted that the errors on the asteroseismic ages are internal errors from the fit. However, even conservatively assuming a precision of $\sim 20 \%$ on the asteroseismic ages (Aerts 2015), the two methods of age estimation are still in poor agreement. 
4 Paper III - Constraining differential rotation of Sun-like stars from asteroseismic and starspot rotation periods

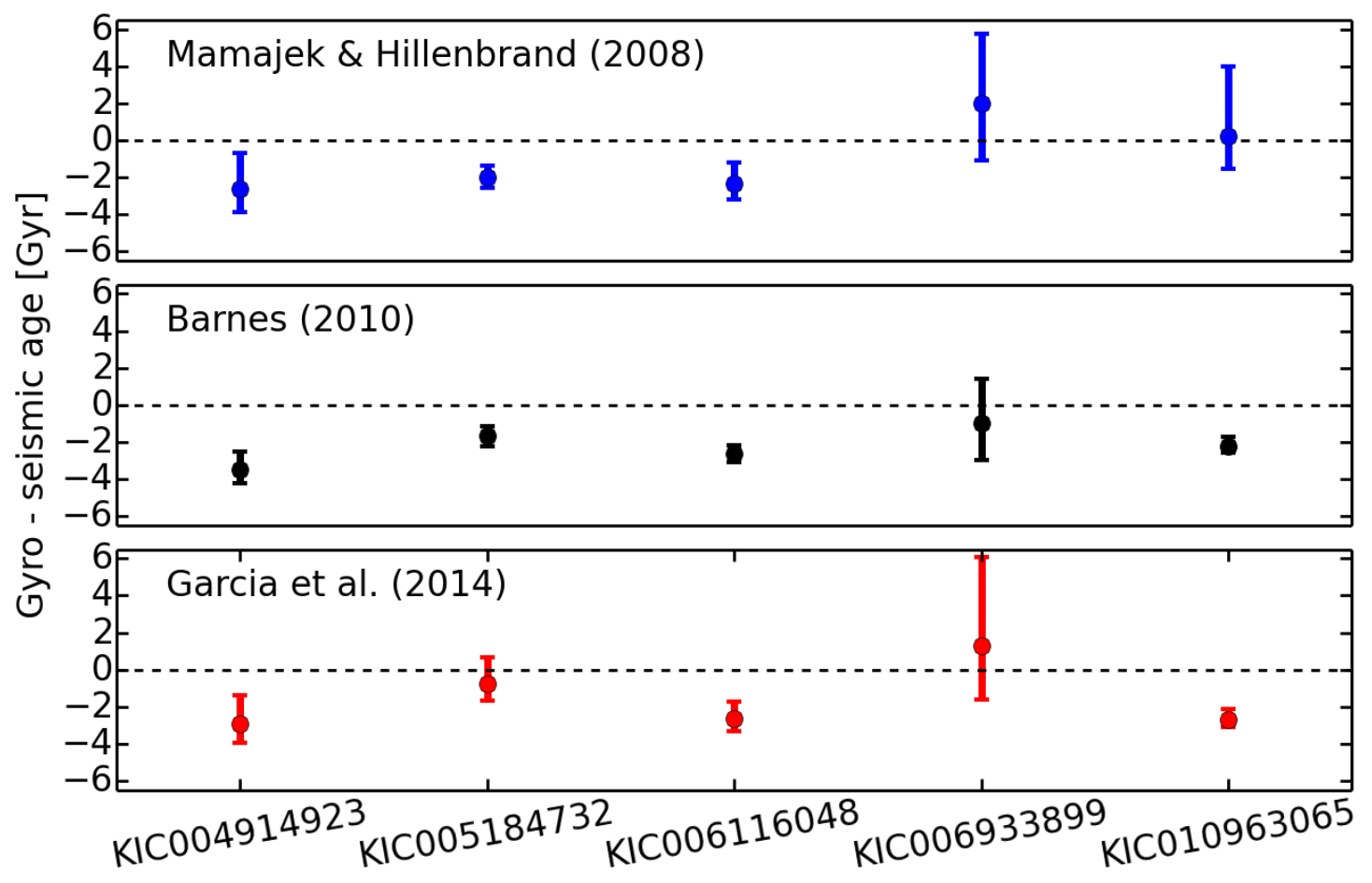

Figure 4.4: Differences between gyrochronology ages and asteroseismic ages for three different gyrochronology relations. The relations are Mamajek and Hillenbrand (2008), Barnes (2010), and García et al. (2014).

\subsection{Conclusions}

We measured the rotation periods of five Sun-like stars, using asteroseismology and surface variability. The measurements show that the asteroseismic rotation periods are $\sim 4 \%$ lower than those from surface variability, but this was not found to be a statistically significant difference given the measurement uncertainties. Thus there is no evidence for any radial (or latitudinal) differential rotation between the regions that the two methods are sensitive to.

Previous efforts to calibrate gyrochronology relations have focused on measuring rotation from surface variability of cluster stars. Given that rotation periods can be reliably determined by asteroseismology compared to the surface variability, gyrochronology may also be applied to very inactive stars for which surface variability rotation periods are not available.

Our comparison of three different gyrochronology relations from the literature found that these ages differed by up to several Gyr from the seismic ages. In general, the gyrorelations seemed to underestimate the stellar ages relative to the asteroseismic ages. It also demonstrated that the gyro-relations are not internally consistent.

When considering these discrepancies it is important to remember the calibrations used for each method. The Barnes and Kim (2010) and Mamajek and Hillenbrand (2008) relations are both calibrated using ages and rotation periods of the Sun and cluster stars, and so may not accurately predict ages of stars similar to or older than the Sun (e.g., Meibom et al. 2015). On the other hand, asteroseismic ages from stellar models are only 
calibrated to the Sun, and in addition are dependent on the input physics and assumptions of the stellar models (Lebreton and Goupil 2014). The relation by García et al. (2014) lacks a mass dependent term, in contrast to the two other tested relations. However, this was not expected to have a remarkable effect since the stars in our sample were approximately $1 \mathrm{M}_{\odot}$ stars, similar to the calibrators used by García et al. (2014). Despite this, there is still a discrepancy of several Gyr between the ages derived from this relation and our seismic ages for some of the stars.

Tighter asteroseismic constraints for stellar modeling will become available when calibrations are obtained from cluster stars to be observed by space missions like K2 (Chaplin et al. 2015) and the Planetary Transits and Oscillations of stars (PLATO) mission (Rauer et al. 2014). This in turn will allow us to reliably use asteroseismic rotation periods to further refine gyrochronology relations for magnetically inactive field stars. 



\section{Discussion: constraining interior rotational shear}

The measurements performed in Chapters 3 and 4 can be used to make more quantitative statements about the internal rotation profile of the studied stars. We determined that the difference in the rotational splittings as a function of frequency is not large, consistent with what would be observed in the Sun if viewed as a star. In addition to this we found that the spot rotation periods are consistent with the seismic rotation periods; showing that any radial or latitudinal differential rotation is likely small. In this section we will use forward modeling with a simple radial step rotation profile and investigate how well the above mentioned measurements can constrain such a model. This will allow us to estimate the upper limits of the radial shear at the tachocline.

\subsection{Modeling radial differential rotation}

The radial step profile that we consider for the radial differential rotation consists of dividing the star into two zones: the radiative interior and the convective envelope, and assuming constant and independent rotation rates for each. Such a rotation profile has the form

$$
\Omega(r)=\left\{\begin{array}{ll}
\Omega_{C} & 0 \leq r \leq r_{c z} \\
\Omega_{E} & r_{c z}<r \leq R
\end{array},\right.
$$

where $\Omega_{\mathrm{C}}$ and $\Omega_{\mathrm{E}}$ are the angular velocities of the radiative interior and convective envelope respectively. The point of separation between the two zones, $r_{\mathrm{cz}}$, is the radius of the base of the convection zone, and $\mathrm{R}$ is the stellar radius; both of these quantities are computed from the stellar models detailed in Chapter 4, and listed in Table 5.1.

The difference of the two rotational velocities $\Omega_{\mathrm{E}}-\Omega_{\mathrm{C}}$ gives some indication about the magnitude of the tachocline shear. This in turn could be used to show to what extent the tachocline might play a role in the generation of stellar magnetic activity, or how well the two zone are able to couple and exchange angular momentum.

Using this simple model together with Eq. 1.1 the rotational splitting of a mode multiplet can be written as

$$
\delta v_{n l m}=m \frac{\Omega_{\mathrm{C}}}{2 \pi} \int_{0}^{r_{c z}} K_{n l}(r) d r+m \frac{\Omega_{\mathrm{E}}}{2 \pi} \int_{r_{c z}}^{R} K_{n l}(r) d r .
$$

Here $K_{n l m}(\theta, r)$ from Eq. 1.2 is reduced to its radial component (see derivation in Christensen- 

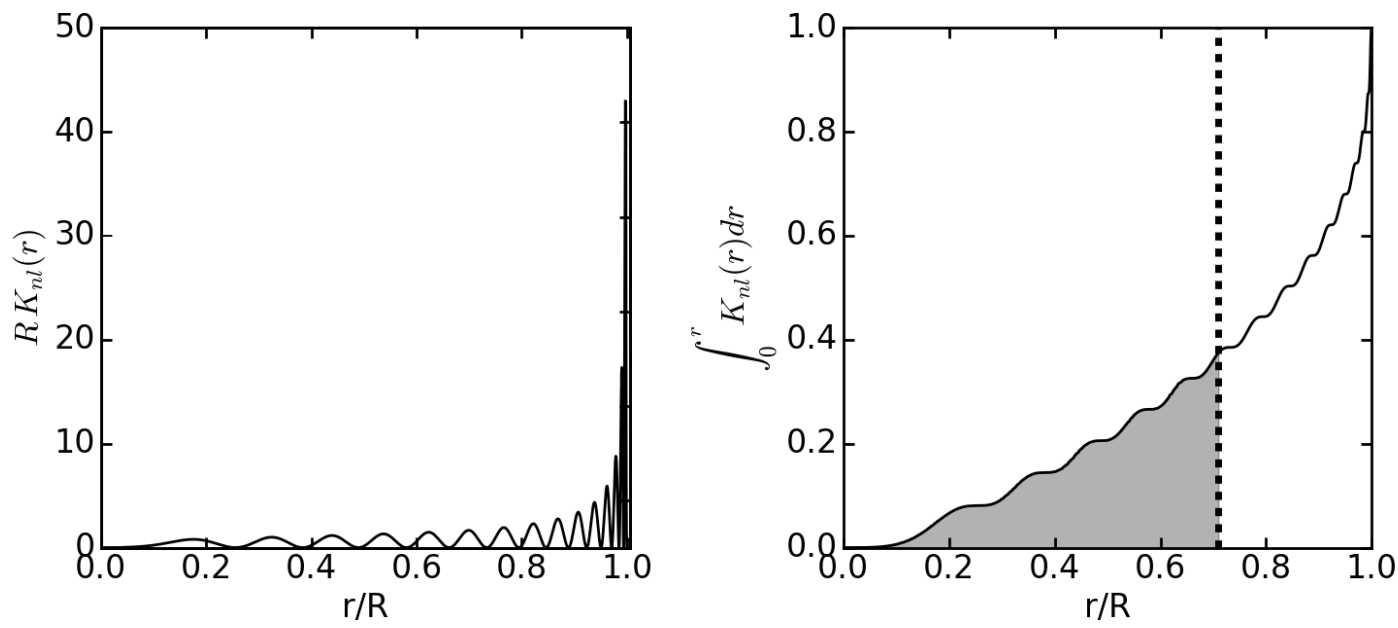

Figure 5.1: Left: The radial component of one of the rotation sensitivity kernels $K_{n l}(r)$ for the star KIC004914923. Right: The cumulative integral of the rotation kernel. The shaded region denotes the integral from the center up to the base of the convection zone (dashed).

Dalsgaard 2003), such that

$$
K_{n l}(r)=\frac{\left(\xi_{r}^{2}+l(l+1) \xi_{h}^{2}-2 \xi_{r} \xi_{h}-\xi_{h}^{2}\right) r^{2} \rho}{\int_{0}^{R}\left(\xi_{r}^{2}+l(l+1) \xi_{r}^{2}\right) r^{2} \rho d r}
$$

where as before $\xi_{r}(r), \xi_{h}(r)$, and $\rho(r)$ are computed using ADIPLS and the stellar structure models from Chapter 4.

The integrals of $K_{n l}(r)$ are constants corresponding to the sensitivity of the modes to rotation in the respective zones, i.e., $\delta v_{n l m}$ becomes a weighted average of the rotation velocities $\Omega_{\mathrm{C}}$ and $\Omega_{\mathrm{E}}$. The left frame in Fig. 5.1 shows $K_{n l}(r)$ for an $l=1$ for KIC004914923, while the right frame shows its cumulative integral. This shows that roughly $40 \%$ of the kernel is located below the convection zone, meaning there is still significant sensitivity to rotation in this region.

\subsubsection{Computing the mode set splittings}

Equation 5.2 provides the rotational splitting for a single multiplet. However, in Chapter 3 we measured a splitting for a mode set consisting of $(n, l=2),(n+1, l=0)$, and $(n+1, l=1)$. Only the $l=2$ and the $l=1$ multiplets feel the rotation, so a splitting for a particular mode set will consist of an average of the two. Since the $S / N$ differs significantly between the modes (see, e.g., Fig. 3.1) this is a weighted average. Any computed model splitting for a given mode set must therefore also be weighted in a similar way. We can therefore write the splitting of a mode set $k$ as

$$
\delta v_{k}=\frac{w_{2} \delta v_{n, l=2}+w_{1} \delta v_{n+1, l=1}}{w_{2}+w_{1}}
$$




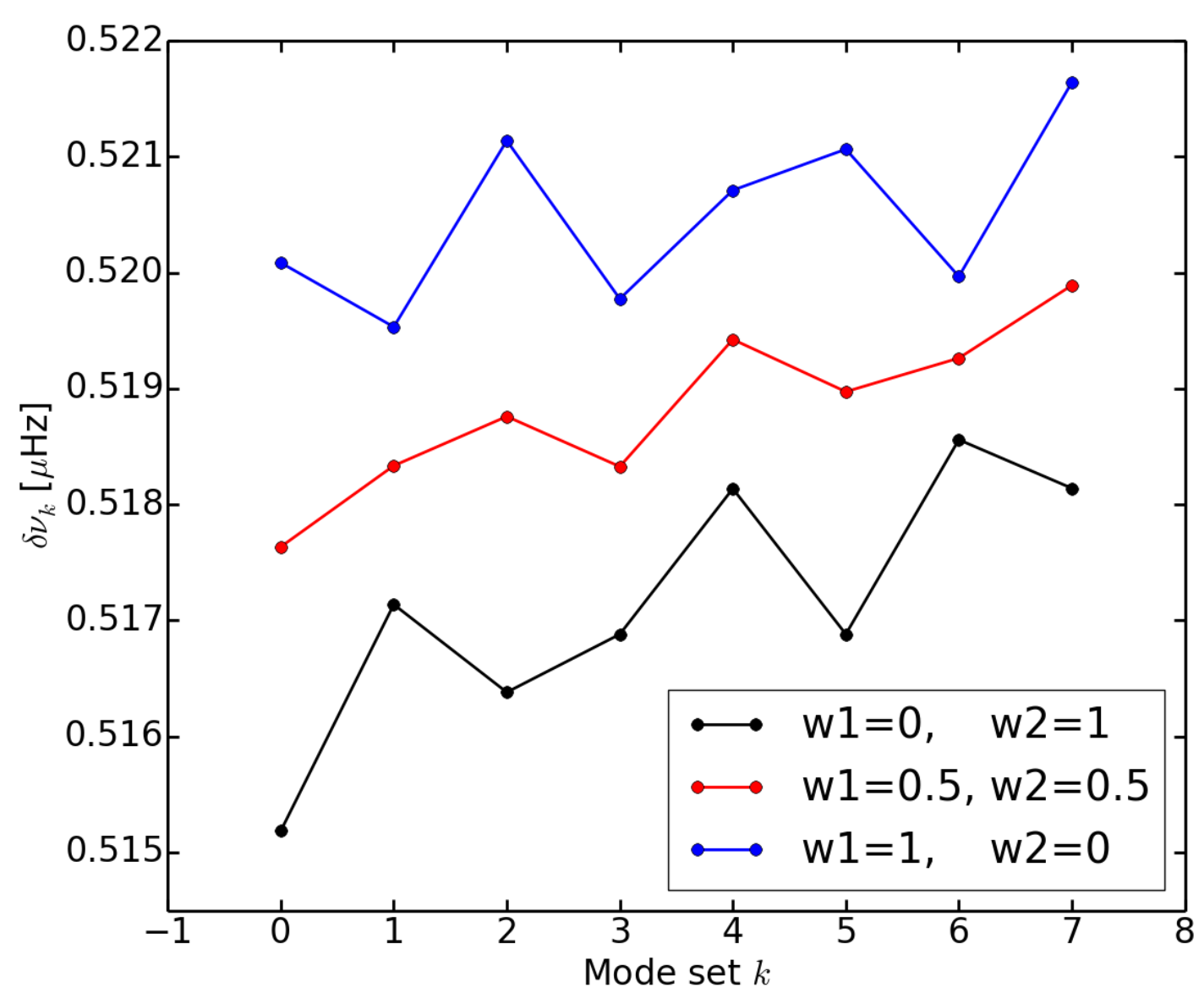

Figure 5.2: Computed rotational splittings using different combinations of the weights $w_{1}$ and $w_{2}$ for the star KIC004914923. The weights are varied such that either $\delta v_{n l=2}$ or $\delta v_{n+1, l=1}$ is weighted completely, as well as even weighting. This translates into a difference of only a few $\mathrm{nHz}$, showing that the mode set splittings are relatively insensitive to the choice of weights on the individual $l=1$ and $l=2$ multiplet splittings, compared to other noise sources.

where $w_{l}$ is the weight for the multiplet $l$ in the mode set $k$.

The weights $w_{l}$ are not straightforward to compute from first principles (Gizon 1996). However, we tested different weights on the individual $l=1$, and $l=2$ splittings and found that the choice of $w_{l}$ ultimately plays a very small role in the variation of the mode set splitting with frequency. Figure 5.2 shows an example of the computed rotational splittings, using a constant rotation profile $\Omega_{\mathrm{C}} / 2 \pi=\Omega_{\mathrm{E}} / 2 \pi=0.53 \mu \mathrm{Hz}$. The weights are varied between complete weighting toward either $\delta v_{n l=2}$ or $\delta v_{n+1, l=1}$, or an even weighting between the two terms. This shows that the differences in the splittings of the $l=1$ and $l=2$ multiplets are on the order of $\sim 5 \mathrm{nHz}$ which is much less than the uncertainties of the measured mode set splittings. For the sake of simplicity we therefore pick the values $w_{1}=0.75$, and $w_{2}=0.25$. 


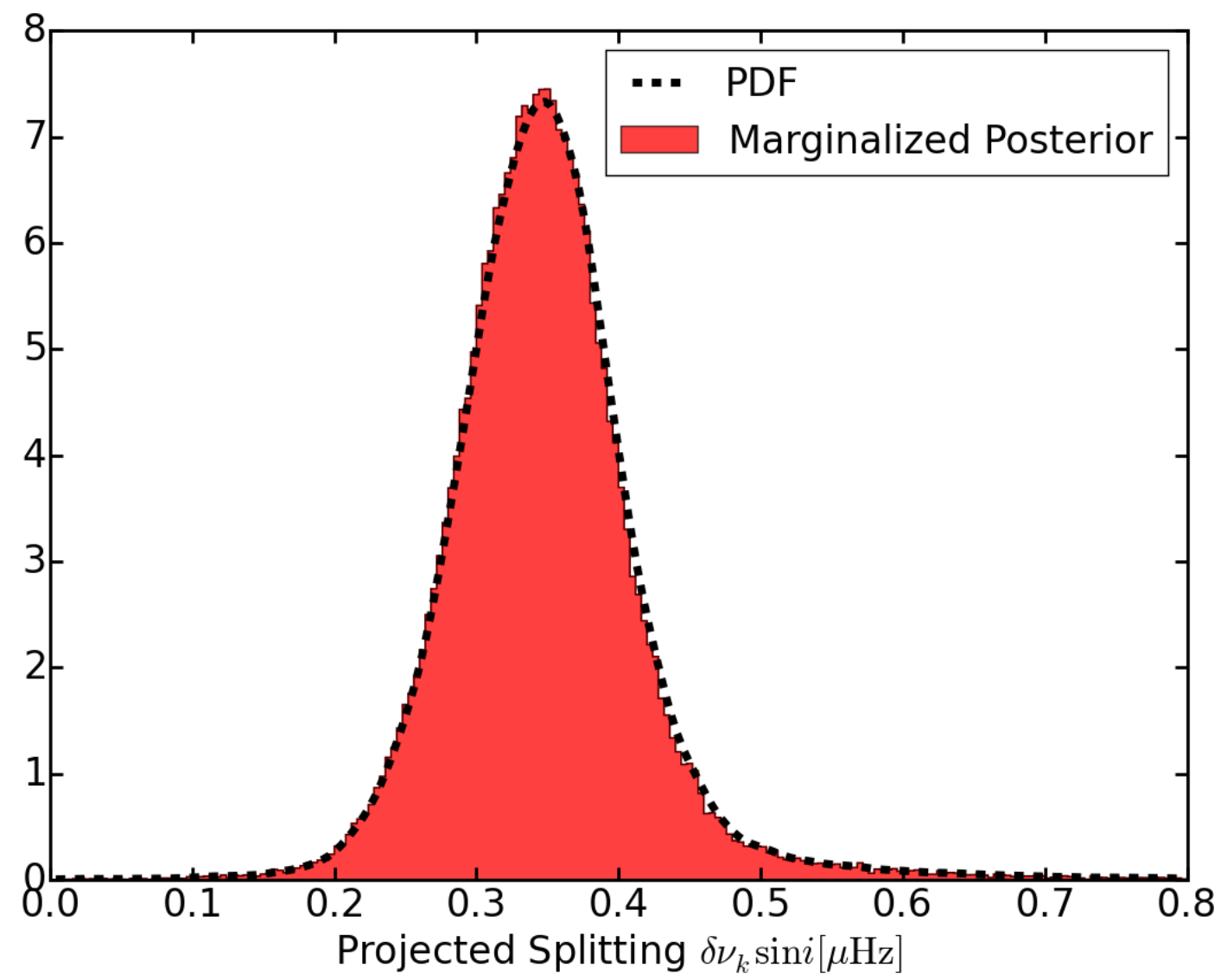

Figure 5.3: The marginalized posterior distribution (red) from KIC004914923 of a mode set $k=3$ at $\sim 1644 \mu \mathrm{Hz}$. The dashed curve shows the smoothed and interpolated histogram of the posterior distribution which is used as a PDF in Eq. 5.5.

\subsubsection{Using only seismic data to constrain the radial shear}

Given the measured mode set splittings from Chapter 3 and using Eq. 5.4 we wish to find the limits of $\Omega_{\mathrm{C}}$ and $\Omega_{\mathrm{E}}$, and thus constrain the radial shear at the base of the convection zone.

How well the splittings constrain the radial shear can be estimated by computing the likelihood of observing a set of splittings like those from Chapter 3, given a particular combination of $\Omega_{\mathrm{C}}$ and $\Omega_{\mathrm{E}}$. The fits to the mode sets in Chapter 3 were performed independently of each other, with only the background term being in common between them, i.e., the splittings are independent. The likelihood is the joint probability of observing each mode set splitting, so we can compute this by

$$
L\left(\Omega_{\mathrm{C}}, \Omega_{\mathrm{E}}\right)=\prod_{k=1}^{N} P_{k}\left(\delta v_{k}\left(\Omega_{\mathrm{C}}, \Omega_{\mathrm{E}}\right)\right),
$$

where $N \approx 6$ is the number of mode sets that were fit for a given star, and $P_{k}\left(\delta v_{k}\left(\Omega_{\mathrm{C}}, \Omega_{\mathrm{E}}\right)\right)$ is the probability of observing a particular splitting given $\Omega_{\mathrm{C}}$ and $\Omega_{\mathrm{E}}$. 


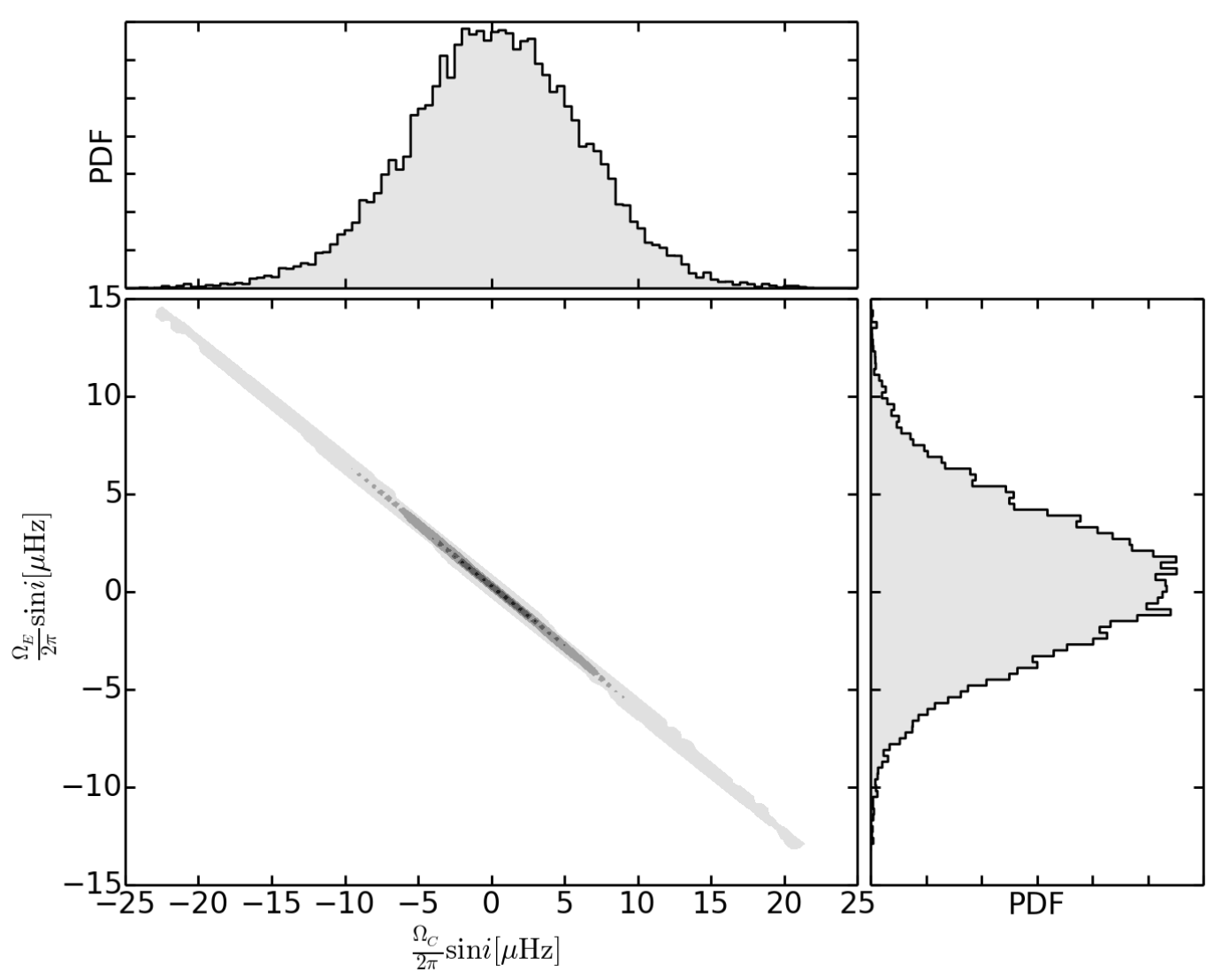

Figure 5.4: Correlation plot of $\Omega_{\mathrm{E}} / 2 \pi \sin i$ and $\Omega_{\mathrm{C}} / 2 \pi \sin i$ for KIC004914923, with the corner plots showing the MPDs of each parameter.

In Chapter 3 we used an MCMC sampler to find the maximum likelihood in the fit to each mode set. The burnt-in chains represent samples drawn from the highest probability region of the posterior distribution of a mode set model, given the power in the corresponding section of the spectrum. Marginalizing over the frequencies, amplitudes, width, and inclination in the fit, produces the marginalized posterior distribution (MPD) of the mode set splitting. We can use this to estimate the PDF $P_{k}\left(\delta v_{k}\right)$ of the splitting for each mode set, by computing a histogram of the sample obtained from the MCMC chain and normalizing this to unit integral.

The errors on the splittings will be the main contributor to the uncertainty on the radial shear. However, as was seen in Fig. 3.3, the rotational splitting of a mode set and the inclination of the stellar rotation axis are highly correlated. Moreover, since the fits to the mode sets were done independently the inclinations were poorly constrained, leading to large errors on the splittings. This can be overcome by using the projected splitting instead, i.e., $\delta v_{k} \sin i$. For the purposes of the present work this translates into simply using $\Omega(r) \sin i$ to compute the splittings from the model. Figure 5.3 shows an example of an MPD for one of the mode sets observed in the power spectrum of KIC006116048 (red), and the resulting PDF in dashed black.

Figure 5.4 shows the likelihoods $L$ for a range of $\Omega_{\mathrm{E}} / 2 \pi \sin i$ and $\Omega_{\mathrm{C}} / 2 \pi \sin i$, along 


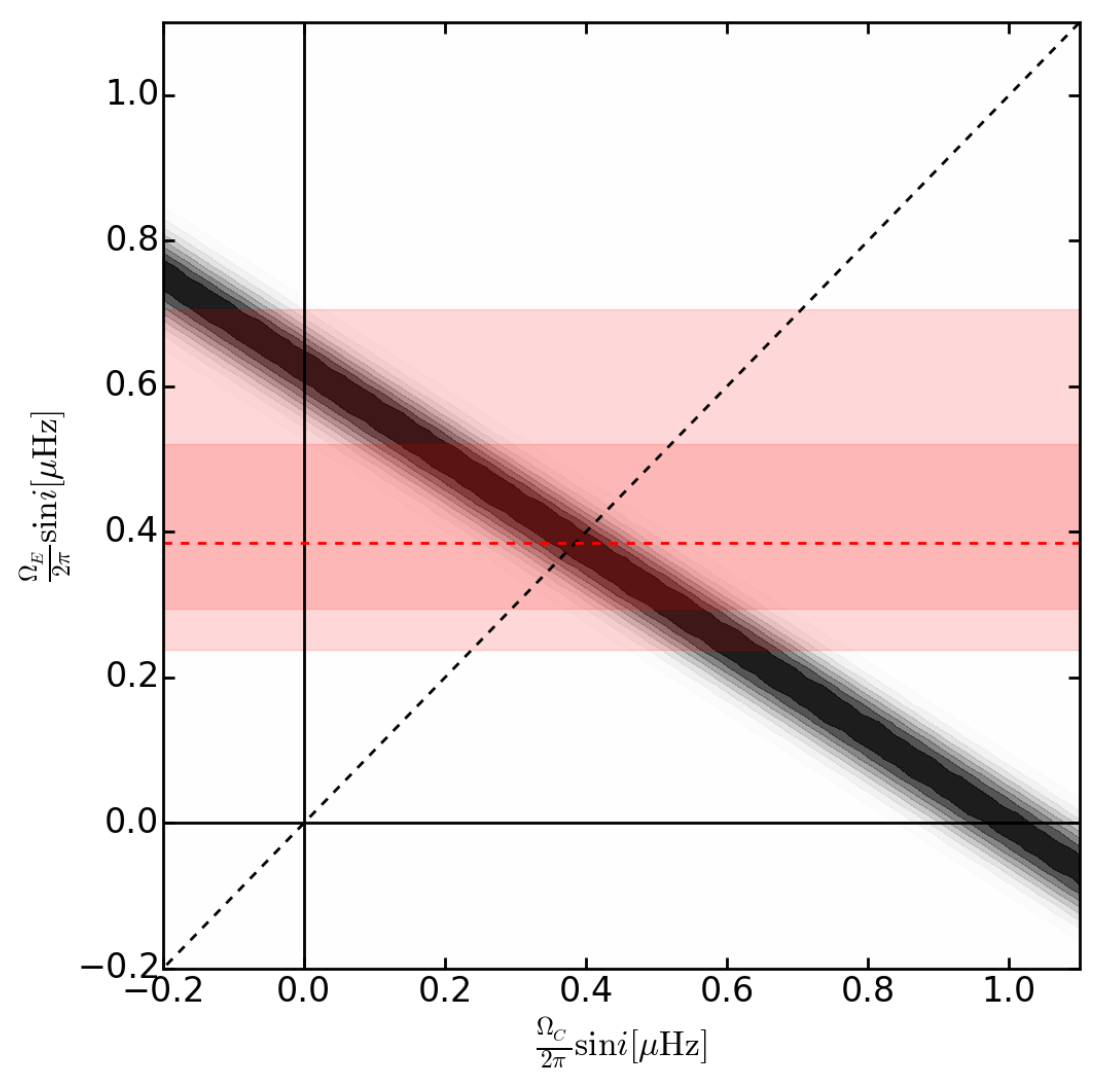

Figure 5.5: A zoom of Fig. 5.4 around the origin for the star KIC004914923. Here the likelihood from equation 5.5 is shown in black. The surface rotation rates from Chapter 4 are plotted in red, with the shade of red denoting the $68 \%$ and $95 \%$ confidence intervals. The dashed line shows solid-body rotation where $\Omega_{\mathrm{E}}=\Omega_{\mathrm{C}}$. The abscissa and ordinate are shown for clarity.

with the MPDs of the two parameters. From the posterior distributions alone it is clear that the two parameters are very poorly constrained. The $2 \sigma$ confidence interval in both cases is on the order of $\pm 10 \mu \mathrm{Hz}$, orders of magnitude higher than the shear scales observed in the Sun. Clearly this does not lead to a meaningful constraint on the radial shear.

The likelihood also shows that the two parameters are very tightly anti-correlated. This means that if a constraint can be placed on $\Omega_{\mathrm{E}} \sin i$ (e.g., from spot rotation) it would in turn also constrain $\Omega_{\mathrm{C}} \sin i$, and thereby the size of the radial shear.

\subsubsection{Using prior information from surface variability}

In Chapter 4 we saw that the rotation rate from surface variability matches that of the average rotation rate of the entire star as measured by asteroseismology. This was also found by Gizon et al. (2013) for the star HD52265, and later confirmed by (Benomar et al. 2015) with $v \sin i$ measurements. For solar-like oscillators the mode sensitivity is weighted toward the envelope and in a band of approximately $\pm 40^{\circ}$ degrees around the 

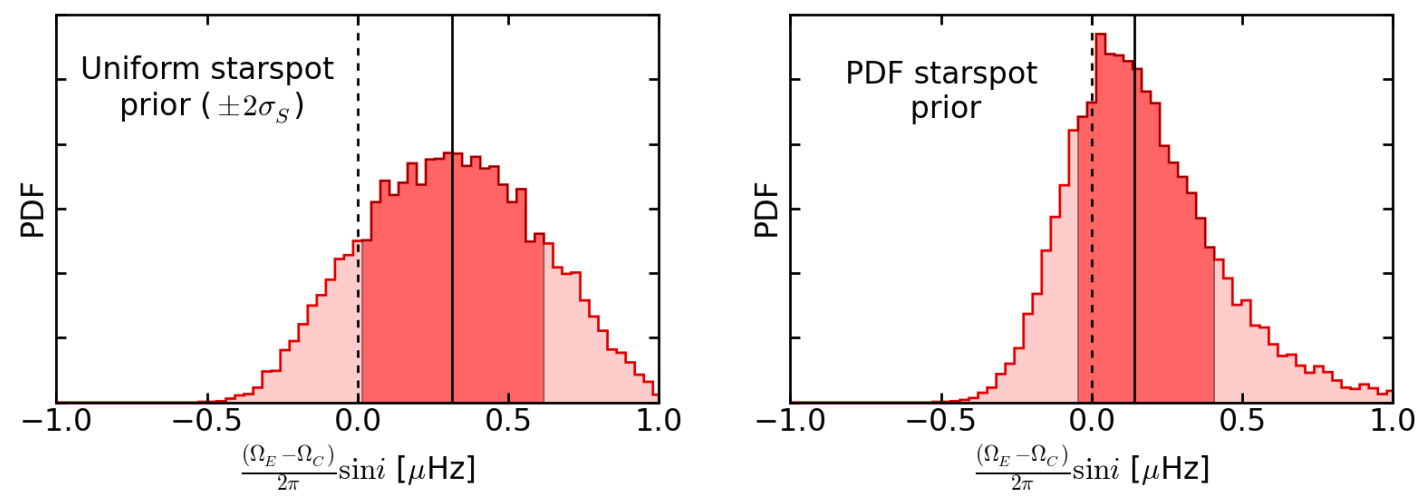

Figure 5.6: Left: Samples drawn from the probability density of $\left(\Omega_{\mathrm{E}}-\Omega_{\mathrm{C}}\right) / 2 \pi \sin i$ for the star KIC004914923, using a uniform spot prior. The dark shaded regions denote the $68 \%$ confidence interval for the distribution, and the solid line denotes the median. The dashed line represents solid-body rotation at $\Omega_{\mathrm{E}}-\Omega_{\mathrm{C}}=0$. Right: Same as the left frame, but using the full PDF of the spot period distribution as a prior.

equator (see Fig 1.8 and, e.g., Lund et al. 2014b, Davies et al. 2015). This means that an average global rotation rate measured by asteroseismology is weighted toward this region. Since the surface rotation rate was found to agree with this weighted average, we can make the assumption that the average envelope rotation rate is in turn not very different. This allows us to use the projected surface rotation rate $\Omega_{\mathrm{S}} \sin i$ in order to constrain $\Omega_{\mathrm{E}} \sin i$, and thereby also $\Omega_{\mathrm{C}} \sin i$.

Note that it is only because of the strong agreement between the average seismic and surface rotation rates, that we can make this assumption. Naturally, this is not a valid assumption for all stars. Many fast rotators are known to have near-polar spots (Strassmeier 2009), and in the presence of strong latitudinal differential rotation, the rotation rate as measured by those spots will likely differ from the average envelope rotation as measured by asteroseismology. Therefore, we start by taking the conservative approach and using the $95 \%$ confidence interval of the projected surface rotation rate $\Omega_{\mathrm{S}} / 2 \pi \sin i$ as a uniform prior on $\Omega_{\mathrm{E}} / 2 \pi \sin i$. In Fig. 5.5 we show a zoom of Fig. 5.4 around the origin, with $\Omega_{\mathrm{S}} / 2 \pi \sin i$ plotted in red, where $\Omega_{\mathrm{S}}$ and $\sin i$ are both obtained from Chapter 4 . The shades of red denote the $68 \%$ and $95 \%$ confidence intervals of $\Omega_{\mathrm{S}} / 2 \pi \sin i$, and dashed black indicates $\Omega_{\mathrm{E}}=\Omega_{\mathrm{C}}$.

The limits of $\Omega_{\mathrm{E}}-\Omega_{\mathrm{C}}$ are found by computing its PDF. This is done by initially computing the joint probability function of the spot distribution and the asteroseismic measurements (red and gray respectively in Fig. 5.5), i.e., the overlapping regions. This provides the most likely solutions for the projected radial shear $\left(\Omega_{\mathrm{E}}-\Omega_{\mathrm{C}}\right) \sin i$. Figure 5.6 shows samples drawn from the PDF of the projected shear for KIC004914923. The left frame shows samples drawn using the uniform prior. This interval is given by $\Omega_{\mathrm{S}} / 2 p i \sin i$ and the corresponding errors listed in Table 5.1. This shows that applying a simple uniform prior to the envelope rotation rate excludes a wide range of possible combinations of $\Omega_{\mathrm{C}}$ and $\Omega_{\mathrm{E}}$.

Alternatively one can instead use the true shape of the $\Omega_{\mathrm{S}} / 2 \pi \sin i$ PDF as a prior. The result of this is shown in the right frame of Fig. 5.6. Using such a prior serves to 
constrain the possible range of $\Omega_{\mathrm{E}}-\Omega_{\mathrm{C}}$ even further, moving it closer to the solid-body configuration. However, this carries with it stronger assumptions about the latitude of the surface features, relative to the latitude corresponding to the mean rotation of the envelope as measured by seismology.

The sin $i$ dependence of the projected shear can be removed by instead considering the relative radial shear $\left(\Omega_{\mathrm{E}}-\Omega_{\mathrm{C}}\right) / \Omega_{\mathrm{E}}$. This uses the assumption that the radiative interior and convective envelope rotate around the same axis. The relative shear for each of the studied stars is show in Fig. 5.7, where we have used the full PDF of $\Omega_{\mathrm{S}} / 2 \pi \sin i$. Table 5.1 lists the median and $68 \%$ confidence interval of these distributions, along with those using a uniform prior.

The measured shear values all show a high degree of symmetry around the median, which tends to lie close $\Omega_{\mathrm{E}}-\Omega_{\mathrm{C}}=0$, i.e., the solid-body configuration is the most likely solution. Furthermore, the widths of the distributions show that in these stars the shear relative to the envelope rotation rate is most likely no greater than $\sim 50 \%$. 

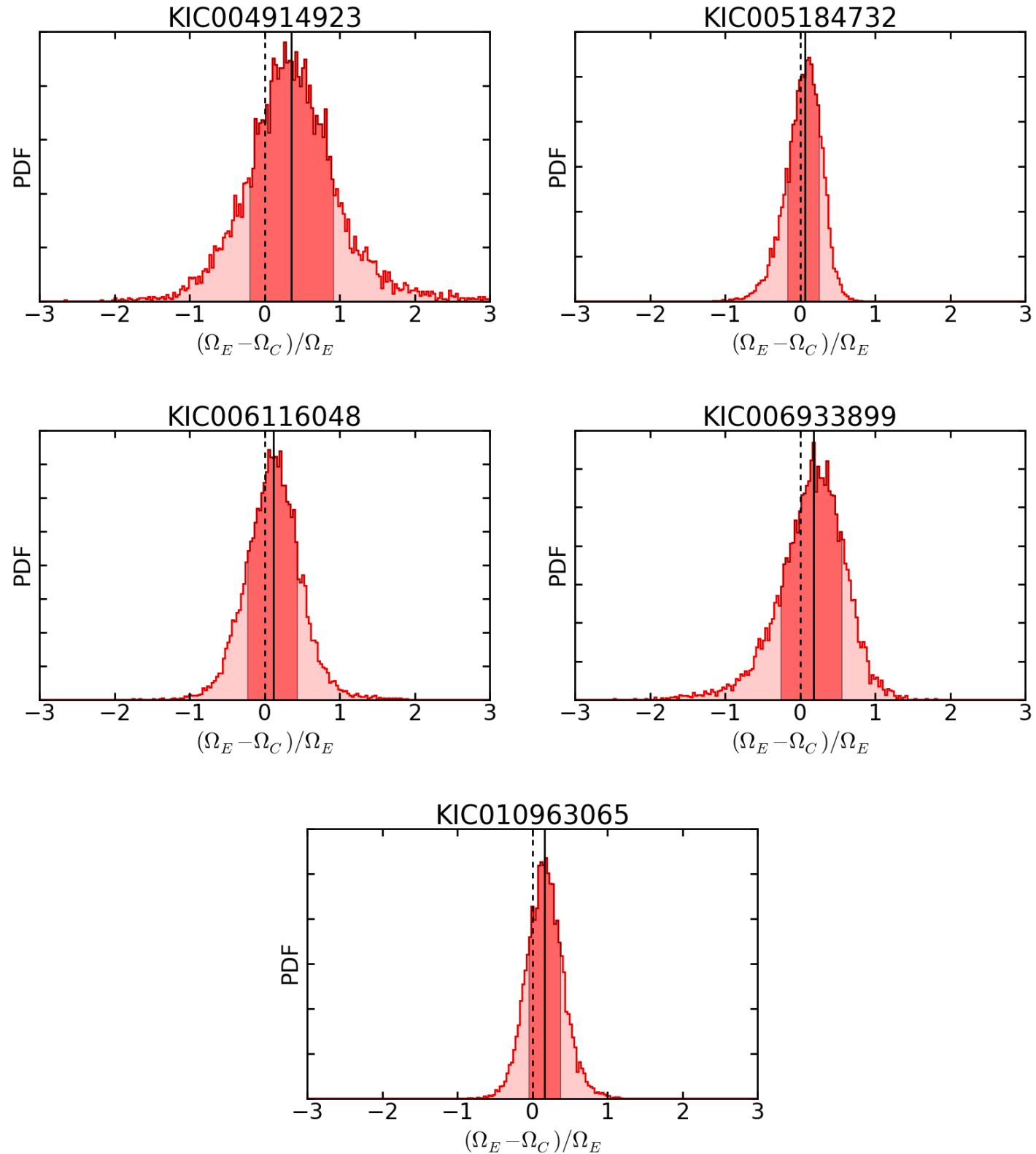

Figure 5.7: The distributions of relative shear $\left(\Omega_{\mathrm{C}}-\Omega_{\mathrm{E}}\right) / \Omega_{\mathrm{C}}$ that are possible for each of the studied stars, using the full PDF of $\Omega_{\mathrm{S}} \sin i$ as a prior. The shaded region denotes the $68 \%$ confidence interval of each distribution, and the solid line is the median. The dashed line represents solid-body rotation. 
Table 5.1: Median values of the projected surface rotation rate $\Omega_{\mathrm{S}} / 2 \pi \sin i$, and relative shear using two different types of priors. The two priors are a uniform prior corresponding to the $95 \%$ confidence interval of $\Omega_{\mathrm{S}} / 2 \pi \sin i$, and the full PDF of the $\Omega_{\mathrm{S}} / 2 \pi \sin i$. The errors on the relative shear values denote the $68 \%$ confidence interval of each parameter. The radius $R$ and convection zone radius $r_{c z}$ of each star is computed from stellar models from Chapter 4.

\begin{tabular}{ccccccc}
\hline Kepler Object & Radius $\left[\mathrm{R}_{\odot}\right]$ & $r_{c z} / R$ & $\frac{\Omega_{\mathrm{S}}}{2 \pi} \sin i[\mu \mathrm{Hz}]$ & $\frac{\Omega_{\mathrm{E}}}{2 \pi} \sin i[\mu \mathrm{Hz}]$ & $\left(\Omega_{\mathrm{E}}-\Omega_{\mathrm{C}}\right) / \Omega_{\mathrm{E}}$ & $\left(\Omega_{\mathrm{E}}-\Omega_{\mathrm{C}}\right) / \Omega_{\mathrm{E}}$ \\
& & & & Seismology alone & Uniform prior & PDF prior \\
KIC004914923 & 1.378 & 0.724 & $0.39_{-0.15}^{+0.32}$ & $0.42_{-0.02}^{+0.02}$ & $0.68_{-0.66}^{+0.62}$ & $0.35_{-0.55}^{+0.57}$ \\
KIC005184732 & 1.342 & 0.731 & $0.51_{-0.11}^{+0.08}$ & $0.55_{-0.02}^{+0.02}$ & $-0.31_{-0.31}^{+0.26}$ & $0.06_{-0.25}^{+0.21}$ \\
KIC006116048 & 1.225 & 0.757 & $0.60_{-0.17}^{+0.27}$ & $0.63_{-0.02}^{+0.02}$ & $0.23_{-0.41}^{+0.36}$ & $0.12_{-0.35}^{+0.34}$ \\
KIC006933899 & 1.574 & 0.786 & $0.30_{-0.10}^{+0.10}$ & $0.34_{-0.03}^{+0.02}$ & $-0.08_{-0.63}^{+0.47}$ & $0.18_{-0.45}^{+0.38}$ \\
KIC010963065 & 1.220 & 0.714 & $0.66_{-0.11}^{+0.11}$ & $0.68_{-0.03}^{+0.03}$ & $0.18_{-0.31}^{+0.28}$ & $0.16_{-0.24}^{+0.24}$ \\
\hline
\end{tabular}




\subsection{Conclusion}

In the work presented here we used a combination of asteroseismic measurements and surface variability, in order to constrain the radial rotational shear inside five Sun-like stars. We assumed that the radial rotation profile can be approximated by independent, constant rotation rates for the radiative interior and convective envelope. The above results show that the rotational splittings found in the previous chapters are consistent with rigid rotation, but also place upper limits on the scale of the radial shear and we find that the relative shear likely does not exceed $\sim 50 \%$. These results show that it is possible to gain insight into the internal rotation of Sun-like stars using the relatively simple procedures described here.

Our ability to measure the internal rotation of Sun-like stars is limited by the $S / N$ in the Kepler observations. The PLATO mission (Rauer et al. 2014) promises to change this. Currently the selection of bright Sun-like stars observed in short cadence by $\mathrm{Ke}$ pler number in the few tens. PLATO on the other hand will perform observations of $\sim 80000$ F-,G-, and K-type stars with magnitudes less than $\sim 11$, i.e., many more than are available in the Kepler seismic catalog. This will provide a much greater selection of bright targets, potentially some that show clear evidence of internal differential rotation. Importantly, it will also be easier to perform ground-based follow-up observations of these stars. Combined with the more precise asteroseismic measurements, this will undoubtedly help complete the picture of stellar rotation and its effects. 



\section{Bibliography}

M. Abramowitz and I. A. Stegun. Handbook of Mathematical Functions. 1972.

C. Aerts. The age and interior rotation of stars from asteroseismology. Astronomische Nachrichten, 336:477, June 2015. doi: 10.1002/asna.201512177.

C. Aerts, A. Thoul, J. Daszyńska, R. Scuflaire, C. Waelkens, M. A. Dupret, E. Niemczura, and A. Noels. Asteroseismology of HD 129929: Core Overshooting and Nonrigid Rotation. Science, 300:1926-1928, June 2003. doi: 10.1126/science.1084993.

C. Aerts, J. Christensen-Dalsgaard, and D. W. Kurtz. Asteroseismology. 2010.

L. Affer, G. Micela, F. Favata, E. Flaccomio, and J. Bouvier. Rotation in NGC 2264: a study based on CoRoT photometric observations. MNRAS, 430:1433-1446, April 2013. doi: 10.1093/mnras/stt003.

S. Aigrain, F. Favata, and G. Gilmore. Characterising stellar micro-variability for planetary transit searches. AEFA, 414:1139-1152, February 2004. doi: 10.1051/0004-6361: 20034039.

S. Aigrain, J. Llama, T. Ceillier, M. L. d. Chagas, J. R. A. Davenport, R. A. García, K. L. Hay, A. F. Lanza, A. McQuillan, T. Mazeh, J. R. de Medeiros, M. B. Nielsen, and T. Reinhold. Testing the recovery of stellar rotation signals from Kepler light curves using a blind hare-and-hounds exercise. MNRAS, 450:3211-3226, July 2015. doi: $10.1093 / \mathrm{mnras} / \mathrm{stv} 853$.

E. R. Anderson, T. L. Duvall, Jr., and S. M. Jefferies. Modeling of solar oscillation power spectra. ApJ, 364:699-705, December 1990. doi: 10.1086/169452.

R. Angus, S. Aigrain, D. Foreman-Mackey, and A. McQuillan. Calibrating gyrochronology using Kepler asteroseismic targets. MNRAS, 450:1787-1798, June 2015. doi: $10.1093 / \mathrm{mnras} / \mathrm{stv} 423$.

T. Appourchaux. On Maximum Likelihood Estimation of averaged power spectra. $A \mathcal{E} A$, 412:903-904, December 2003. doi: 10.1051/0004-6361:20034401.

T. Appourchaux, E. Michel, M. Auvergne, A. Baglin, T. Toutain, F. Baudin, O. Benomar, W. J. Chaplin, S. Deheuvels, R. Samadi, G. A. Verner, P. Boumier, R. A. García, B. Mosser, J.-C. Hulot, J. Ballot, C. Barban, Y. Elsworth, S. J. Jiménez-Reyes, H. Kjeldsen, C. Régulo, and I. W. Roxburgh. CoRoT sounds the stars: p-mode parameters of Sun-like oscillations on HD 49933. AEFA, 488:705-714, September 2008. doi: 10.1051/0004-6361:200810297. 
T. Appourchaux, H. M. Antia, W. Ball, O. Creevey, Y. Lebreton, K. Verma, S. Vorontsov, T. L. Campante, G. R. Davies, P. Gaulme, C. Régulo, E. Horch, S. Howell, M. Everett, D. Ciardi, L. Fossati, A. Miglio, J. Montalbán, W. J. Chaplin, R. A. García, and L. Gizon. A seismic and gravitationally bound double star observed by Kepler. Implication for the presence of a convective core. $A \mathcal{E} A$ A, 582:A25, October 2015. doi: 10.1051/0004-6361/201526610.

S. L. Baliunas, R. A. Donahue, W. H. Soon, J. H. Horne, J. Frazer, L. Woodard-Eklund, M. Bradford, L. M. Rao, O. C. Wilson, Q. Zhang, W. Bennett, J. Briggs, S. M. Carroll, D. K. Duncan, D. Figueroa, H. H. Lanning, T. Misch, J. Mueller, R. W. Noyes, D. Poppe, A. C. Porter, C. R. Robinson, J. Russell, J. C. Shelton, T. Soyumer, A. H. Vaughan, and J. H. Whitney. Chromospheric variations in main-sequence stars. ApJ, 438:269-287, January 1995. doi: 10.1086/175072.

W. H. Ball and L. Gizon. A new correction of stellar oscillation frequencies for nearsurface effects. AEFA, 568:A123, August 2014. doi: 10.1051/0004-6361/201424325.

A. Barekat, J. Schou, and L. Gizon. The radial gradient of the near-surface shear layer of the Sun. AEFA, 570:L12, October 2014. doi: 10.1051/0004-6361/201424839.

J. R. Barnes, A. Collier Cameron, J.-F. Donati, D. J. James, S. C. Marsden, and P. Petit. The dependence of differential rotation on temperature and rotation. MNRAS, 357: L1-L5, February 2005. doi: 10.1111/j.1745-3933.2005.08587.x.

S. A. Barnes. On the Rotational Evolution of Solar- and Late-Type Stars, Its Magnetic Origins, and the Possibility of Stellar Gyrochronology. ApJ, 586:464-479, March 2003. doi: $10.1086 / 367639$.

S. A. Barnes. Ages for Illustrative Field Stars Using Gyrochronology: Viability, Limitations, and Errors. ApJ, 669:1167-1189, November 2007. doi: 10.1086/519295.

S. A. Barnes. A Simple Nonlinear Model for the Rotation of Main-sequence Cool Stars. I. Introduction, Implications for Gyrochronology, and Color-Period Diagrams. ApJ, 722 : 222-234, October 2010. doi: 10.1088/0004-637X/722/1/222.

S. A. Barnes and Y.-C. Kim. Angular Momentum Loss from Cool Stars: An Empirical Expression and Connection to Stellar Activity. ApJ, 721:675, September 2010. doi: 10.1088/0004-637X/721/1/675.

S. A. Barnes, J. Weingrill, T. Granzer, F. Spada, and K. G. Strassmeier. A color-period diagram for the open cluster M 48 (NGC 2548), and its rotational age. AEFA, 583:A73, November 2015. doi: 10.1051/0004-6361/201526129.

S. Basu and H. M. Antia. Changes in Solar Dynamics from 1995 to 2002. ApJ, 585: 553-565, March 2003. doi: 10.1086/346020.

M. Bazot, F. Bouchy, H. Kjeldsen, S. Charpinet, M. Laymand, and S. Vauclair. Asteroseismology of $\alpha$ Centauri A. Evidence of rotational splitting. AESA, 470:295-302, July 2007. doi: 10.1051/0004-6361:20065694. 
J. G. Beck. A comparison of differential rotation measurements - (Invited Review). Sol. Phys., 191:47-70, January 2000. doi: 10.1023/A:1005226402796.

P. G. Beck, J. Montalban, T. Kallinger, J. De Ridder, C. Aerts, R. A. García, S. Hekker, M.-A. Dupret, B. Mosser, P. Eggenberger, D. Stello, Y. Elsworth, S. Frandsen, F. Carrier, M. Hillen, M. Gruberbauer, J. Christensen-Dalsgaard, A. Miglio, M. Valentini, T. R. Bedding, H. Kjeldsen, F. R. Girouard, J. R. Hall, and K. A. Ibrahim. Fast core rotation in red-giant stars as revealed by gravity-dominated mixed modes. Nature, 481 : 55-57, January 2012. doi: 10.1038/nature10612.

O. Benomar, M. Takata, H. Shibahashi, T. Ceillier, and R. A. García. Nearly uniform internal rotation of solar-like main-sequence stars revealed by space-based asteroseismology and spectroscopic measurements. MNRAS, 452:2654-2674, September 2015. doi: $10.1093 / \mathrm{mnras} / \mathrm{stv} 1493$.

S. V. Berdyugina. Starspots: A Key to the Stellar Dynamo. Living Reviews in Solar Physics, 2:8, December 2005. doi: 10.12942/lrsp-2005-8.

E. Böhm-Vitense. Chromospheric Activity in G and K Main-Sequence Stars, and What It Tells Us about Stellar Dynamos. ApJ, 657:486-493, March 2007. doi: 10.1086/510482.

W. J. Borucki, D. G. Koch, E. W. Dunham, and J. M. Jenkins. The Kepler Mission: A Mission To Detennine The Frequency Of Inner Planets Near The Habitable Zone For A Wide Range Of Stars. In D. Soderblom, editor, Planets Beyond the Solar System and the Next Generation of Space Missions, volume 119 of Astronomical Society of the Pacific Conference Series, page 153, 1997.

W. J. Borucki, D. Koch, G. Basri, N. Batalha, T. Brown, D. Caldwell, J. Caldwell, J. Christensen-Dalsgaard, W. D. Cochran, E. DeVore, E. W. Dunham, A. K. Dupree, T. N. Gautier, J. C. Geary, R. Gilliland, A. Gould, S. B. Howell, J. M. Jenkins, Y. Kondo, D. W. Latham, G. W. Marcy, S. Meibom, H. Kjeldsen, J. J. Lissauer, D. G. Monet, D. Morrison, D. Sasselov, J. Tarter, A. Boss, D. Brownlee, T. Owen, D. Buzasi, D. Charbonneau, L. Doyle, J. Fortney, E. B. Ford, M. J. Holman, S. Seager, J. H. Steffen, W. F. Welsh, J. Rowe, H. Anderson, L. Buchhave, D. Ciardi, L. Walkowicz, W. Sherry, E. Horch, H. Isaacson, M. E. Everett, D. Fischer, G. Torres, J. A. Johnson, M. Endl, P. MacQueen, S. T. Bryson, J. Dotson, M. Haas, J. Kolodziejczak, J. Van Cleve, H. Chandrasekaran, J. D. Twicken, E. V. Quintana, B. D. Clarke, C. Allen, J. Li, H. Wu, P. Tenenbaum, E. Verner, F. Bruhweiler, J. Barnes, and A. Prsa. Kepler PlanetDetection Mission: Introduction and First Results. Science, 327:977-, February 2010. doi: $10.1126 /$ science.1185402.

J. Bouvier, S. P. Matt, S. Mohanty, A. Scholz, K. G. Stassun, and C. Zanni. Angular Momentum Evolution of Young Low-Mass Stars and Brown Dwarfs: Observations and Theory. Protostars and Planets VI, pages 433-450, 2014. doi: 10.2458/azu_uapress_ 9780816531240-ch019.

T. D. Brandt and C. X. Huang. Rotating Stellar Models Can Account for the Extended Main-sequence Turnoffs in Intermediate-age Clusters. ApJ, 807:25, July 2015. doi: 10.1088/0004-637X/807/1/25. 
M. Briquet, T. Morel, A. Thoul, R. Scuflaire, A. Miglio, J. Montalbán, M.-A. Dupret, and C. Aerts. An asteroseismic study of the $\beta$ Cephei star $\theta$ Ophiuchi: constraints on global stellar parameters and core overshooting. MNRAS, 381:1482-1488, November 2007. doi: 10.1111/j.1365-2966.2007.12142.x.

T. M. Brown, D. W. Latham, M. E. Everett, and G. A. Esquerdo. Kepler Input Catalog: Photometric Calibration and Stellar Classification. AJ, 142:112, October 2011. doi: 10.1088/0004-6256/142/4/112.

H. Bruntt, S. Basu, B. Smalley, W. J. Chaplin, G. A. Verner, T. R. Bedding, C. Catala, J.-C. Gazzano, J. Molenda-Żakowicz, A. O. Thygesen, K. Uytterhoeven, S. Hekker, D. Huber, C. Karoff, S. Mathur, B. Mosser, T. Appourchaux, T. L. Campante, Y. Elsworth, R. A. García, R. Handberg, T. S. Metcalfe, P.-O. Quirion, C. Régulo, I. W. Roxburgh, D. Stello, J. Christensen-Dalsgaard, S. D. Kawaler, H. Kjeldsen, R. L. Morris, E. V. Quintana, and D. T. Sanderfer. Accurate fundamental parameters and detailed abundance patterns from spectroscopy of 93 solar-type Kepler targets. MNRAS, 423:122131, June 2012. doi: 10.1111/j.1365-2966.2012.20686.x.

M. Camenzind. Magnetized Disk-Winds and the Origin of Bipolar Outflows. In G. Klare, editor, Reviews in Modern Astronomy, volume 3 of Reviews in Modern Astronomy, pages 234-265, 1990.

M. Cantiello, C. Mankovich, L. Bildsten, J. Christensen-Dalsgaard, and B. Paxton. Angular Momentum Transport within Evolved Low-mass Stars. ApJ, 788:93, June 2014. doi: 10.1088/0004-637X/788/1/93.

W. J. Chaplin, Y. Elsworth, R. Howe, G. R. Isaak, C. P. McLeod, B. A. Miller, H. B. van der Raay, S. J. Wheeler, and R. New. BiSON Performance. Sol. Phys., 168:1-18, September 1996. doi: 10.1007/BF00145821.

W. J. Chaplin, Y. Elsworth, G. R. Isaak, R. Lines, C. P. McLeod, B. A. Miller, and R. New. Solar p-mode excitation: further insight from recent low-1 BiSON helioseismological data. MNRAS, 298:L7-L12, July 1998. doi: 10.1046/j.1365-8711.1998.01800.x.

W. J. Chaplin, T. R. Bedding, A. Bonanno, A.-M. Broomhall, R. A. García, S. Hekker, D. Huber, G. A. Verner, S. Basu, Y. Elsworth, G. Houdek, S. Mathur, B. Mosser, R. New, I. R. Stevens, T. Appourchaux, C. Karoff, T. S. Metcalfe, J. MolendaŻakowicz, M. J. P. F. G. Monteiro, M. J. Thompson, J. Christensen-Dalsgaard, R. L. Gilliland, S. D. Kawaler, H. Kjeldsen, J. Ballot, O. Benomar, E. Corsaro, T. L. Campante, P. Gaulme, S. J. Hale, R. Handberg, E. Jarvis, C. Régulo, I. W. Roxburgh, D. Salabert, D. Stello, F. Mullally, J. Li, and W. Wohler. Evidence for the Impact of Stellar Activity on the Detectability of Solar-like Oscillations Observed by Kepler. ApJ, 732: L5, May 2011. doi: 10.1088/2041-8205/732/1/L5.

W. J. Chaplin, M. N. Lund, R. Handberg, S. Basu, L. A. Buchhave, T. L. Campante, G. R. Davies, D. Huber, D. W. Latham, C. A. Latham, A. Serenelli, H. M. Antia, T. Appourchaux, W. H. Ball, O. Benomar, L. Casagrande, J. Christensen-Dalsgaard, H. R. Coelho, O. L. Creevey, Y. Elsworth, R. A. Garc, P. Gaulme, S. Hekker, T. Kallinger, C. Karoff, S. D. Kawaler, H. Kjeldsen, M. S. Lundkvist, F. Marcadon, S. Mathur, 
A. Miglio, B. Mosser, C. R, I. W. Roxburgh, V. Silva Aguirre, D. Stello, K. Verma, T. R. White, T. R. Bedding, T. Barclay, D. L. Buzasi, S. Deheuvels, L. Gizon, G. Houdek, S. B. Howell, D. Salabert, and D. R. Soderblom. Asteroseismology of solar-type stars with K2. ArXiv e-prints 1503.06690, Accepted for publication in PASP, July 2015.

P. Charbonneau. Dynamo Models of the Solar Cycle. Living Reviews in Solar Physics, 7 , September 2010. doi: 10.12942/lrsp-2010-3.

S. Charpinet, G. Fontaine, and P. Brassard. Seismic evidence for the loss of stellar angular momentum before the white-dwarf stage. Nature, 461:501-503, September 2009. doi: 10.1038/nature08307.

J. Christensen-Dalsgaard. Lecture Notes on Stellar Oscillations. Institut for Fysik og Astronomi, Aarhus Universitet, and Teoretisk Astrofysik Center, Danmarks Grundforskningsfond, 2003. URL http: //users-phys . au.dk/jcd/oscilnotes/.

J. Christensen-Dalsgaard. ADIPLS-the Aarhus adiabatic oscillation package. ApEsSS, 316:113-120, August 2008. doi: 10.1007/s10509-007-9689-z.

J. L. Christiansen, J. E. Van Cleve, J. M. Jenkins, D. A. Caldwell, T. Barclay, S. T. Bryson, C. J. Burke, B. D. Clarke, M. T. Cote, F. Girouard, M. R. Haas, J. R. Hall, K. Ibrahim, K. Kinemuchi, T. C. Klaus, J. J. Kolodziejczak, J. Li, S. D. McCauliff, R. Morris, F. Mullally, E. V. Quintana, J. Rowe, S. Seader, J. C. Smith, M. D. Still, M. C. Stumpe, Tenenbaum P. G., S. E. Thompson, J. D. Twicken, and A. K. Uddin. Kepler Data Characteristics Handbook (KSCI-19040-003), 2011.

J. L. Christiansen, J. M. Jenkins, D. A. Caldwell, T. Barclay, S. T. Bryson, C. J. Burke, J. Campbell, J. Catanzarite, B. D. Clarke, J. L. Coughlin, F. Girouard, M. R. Haas, K. Ibrahim, T. C. Klaus, J. J. Kolodziejczak, J. Li, S. D. McCauliff, R. L. Morris, F. Mullally, E. V. Quintana, J. Rowe, A. Sabale, S. Seader, J. C. Smith, M. D. Still, P. G. Tenenbaum, S. E. Thompson, J. D. Twicken, and A. K. Uddin. Kepler Data Characteristics Handbook (KSCI - 19040 - 004), 2013.

A. Collier Cameron. Differential rotation on rapidly rotating stars. Astronomische Nachrichten, 328:1030, December 2007. doi: 10.1002/asna.200710880.

M. K. Cowles and B. P. Carlin. Markov chain monte carlo convergence diagnostics: A comparative review. Journal of the American Statistical Association, 91(434):883-904, 1996. ISSN 01621459. URL http: //www. jstor.org/stable/2291683.

G. R. Davies, W. J. Chaplin, W. M. Farr, R. A. García, M. N. Lund, S. Mathis, T. S. Metcalfe, T. Appourchaux, S. Basu, O. Benomar, T. L. Campante, T. Ceillier, Y. Elsworth, R. Handberg, D. Salabert, and D. Stello. Asteroseismic inference on rotation, gyrochronology and planetary system dynamics of 16 Cygni. MNRAS, 446:2959-2966, January 2015. doi: 10.1093/mnras/stu2331.

J. Debosscher, J. Blomme, C. Aerts, and J. De Ridder. Global stellar variability study in the field-of-view of the Kepler satellite. AEFA, 529:A89, May 2011. doi: 10.1051/ 0004-6361/201015647. 
S. Deheuvels, R. A. García, W. J. Chaplin, S. Basu, H. M. Antia, T. Appourchaux, O. Benomar, G. R. Davies, Y. Elsworth, L. Gizon, M. J. Goupil, D. R. Reese, C. Regulo, J. Schou, T. Stahn, L. Casagrande, J. Christensen-Dalsgaard, D. Fischer, S. Hekker, H. Kjeldsen, S. Mathur, B. Mosser, M. Pinsonneault, J. Valenti, J. L. Christiansen, K. Kinemuchi, and F. Mullally. Seismic Evidence for a Rapidly Rotating Core in a Lower-giant-branch Star Observed with Kepler. ApJ, 756:19, September 2012. doi: 10.1088/0004-637X/756/1/19.

S. Deheuvels, G. Doğan, M. J. Goupil, T. Appourchaux, O. Benomar, H. Bruntt, T. L. Campante, L. Casagrande, T. Ceillier, G. R. Davies, P. De Cat, J. N. Fu, R. A. García, A. Lobel, B. Mosser, D. R. Reese, C. Regulo, J. Schou, T. Stahn, A. O. Thygesen, X. H. Yang, W. J. Chaplin, J. Christensen-Dalsgaard, P. Eggenberger, L. Gizon, S. Mathis, J. Molenda-Żakowicz, and M. Pinsonneault. Seismic constraints on the radial dependence of the internal rotation profiles of six Kepler subgiants and young red giants. $A \mathcal{E} A$, 564:A27, April 2014. doi: 10.1051/0004-6361/201322779.

P. Delorme, A. Collier Cameron, L. Hebb, J. Rostron, T. A. Lister, A. J. Norton, D. Pollacco, and R. G. West. Stellar rotation in the Hyades and Praesepe: gyrochronology and braking time-scale. MNRAS, 413:2218-2234, May 2011. doi: 10.1111/j.1365-2966.2011.18299.x.

J.-D. do Nascimento, Jr., R. A. García, S. Mathur, F. Anthony, S. A. Barnes, S. Meibom, J. S. da Costa, M. Castro, D. Salabert, and T. Ceillier. Rotation Periods and Ages of Solar Analogs and Solar Twins Revealed by the Kepler Mission. ApJ, 790:L23, August 2014. doi: 10.1088/2041-8205/790/2/L23.

V. Domingo, B. Fleck, and A. I. Poland. The SOHO Mission: an Overview. Sol. Phys., 162:1-37, December 1995. doi: 10.1007/BF00733425.

J.-F. Donati and A. Collier Cameron. Differential rotation and magnetic polarity patterns on AB Doradus. MNRAS, 291:1-19, October 1997. doi: 10.1093/mnras/291.1.1.

J.-F. Donati, A. Collier Cameron, M. Semel, G. A. J. Hussain, P. Petit, B. D. Carter, S. C. Marsden, M. Mengel, A. López Ariste, S. V. Jeffers, and D. E. Rees. Dynamo processes and activity cycles of the active stars AB Doradus, LQ Hydrae and HR 1099. MNRAS, 345:1145-1186, November 2003. doi: 10.1046/j.1365-2966.2003.07031.x.

J.-F. Donati, C. Moutou, R. Farès, D. Bohlender, C. Catala, M. Deleuil, E. Shkolnik, A. Collier Cameron, M. M. Jardine, and G. A. H. Walker. Magnetic cycles of the planet-hosting star $\tau$ Bootis. MNRAS, 385:1179-1185, April 2008. doi: 10.1111/j. 1365-2966.2008.12946.x.

S. D'Silva and R. F. Howard. Sunspot rotation and the field strengths of subsurface flux tubes. Sol. Phys., 151:213-230, May 1994. doi: 10.1007/BF00679072.

B. R. Durney, D. Mihalas, and R. D. Robinson. A preliminary interpretation of stellar chromospheric CA II emission variations within the framework of stellar dynamo theory. PASP, 93:537-543, October 1981. doi: 10.1086/130878. 
T. L. Duvall, Jr. and J. W. Harvey. Solar Doppler shifts: Sources of continuous spectra. In D. O. Gough, editor, NATO Advanced Science Institutes (ASI) Series C, volume 169 of NATO Advanced Science Institutes (ASI) Series C, pages 105-116, 1986.

P. Eggenberger, J. Montalbán, and A. Miglio. Angular momentum transport in stellar interiors constrained by rotational splittings of mixed modes in red giants. AEFA, 544: L4, August 2012. doi: 10.1051/0004-6361/201219729.

R. Fares, J.-F. Donati, C. Moutou, D. Bohlender, C. Catala, M. Deleuil, E. Shkolnik, A. Collier Cameron, M. M. Jardine, and G. A. H. Walker. Magnetic cycles of the planet-hosting star $\tau$ Bootis - II. A second magnetic polarity reversal. MNRAS, 398: 1383-1391, September 2009. doi: 10.1111/j.1365-2966.2009.15303.x.

D. Foreman-Mackey, D. W. Hogg, D. Lang, and J. Goodman. emcee: The MCMC Hammer. PASP, 125:306-312, March 2013. doi: 10.1086/670067.

S. Frandsen, A. Jones, H. Kjeldsen, M. Viskum, J. Hjorth, N. H. Andersen, and B. Thomsen. CCD photometry of the $\delta$-Scuti star $\kappa^{\wedge} 2^{\wedge}$ Bootis. AEFA, 301:123, September 1995.

M. Fridlund, A. Baglin, J. Lochard, and L. Conroy, editors. The CoRoT Mission PreLaunch Status - Stellar Seismology and Planet Finding, volume 1306 of ESA Special Publication, November 2006.

C. Frohlich, B. N. Andersen, T. Appourchaux, G. Berthomieu, D. A. Crommelynck, V. Domingo, A. Fichot, W. Finsterle, M. F. Gomez, D. Gough, A. Jimenez, T. Leifsen, M. Lombaerts, J. M. Pap, J. Provost, T. R. Cortes, J. Romero, H. Roth, T. Sekii, U. Telljohann, T. Toutain, and C. Wehrli. First Results from VIRGO, the Experiment for Helioseismology and Solar Irradiance Monitoring on SOHO. Sol. Phys., 170:1-25, 1997. doi: 10.1023/A:1004969622753.

H.-E. Fröhlich, M. Küker, A. P. Hatzes, and K. G. Strassmeier. On the differential rotation of CoRoT-2a. AEFA, 506:263-268, October 2009. doi: 10.1051/0004-6361/200911895.

R. A. García, T. Ceillier, D. Salabert, S. Mathur, J. L. van Saders, M. Pinsonneault, J. Ballot, P. G. Beck, S. Bloemen, T. L. Campante, G. R. Davies, J.-D. do Nascimento, Jr., S. Mathis, T. S. Metcalfe, M. B. Nielsen, J. C. Suárez, W. J. Chaplin, A. Jiménez, and C. Karoff. Rotation and magnetism of Kepler pulsating solar-like stars. Towards asteroseismically calibrated age-rotation relations. AEFA, 572:A34, December 2014. doi: 10.1051/0004-6361/201423888.

S. Geman and D. Geman. Stochastic relaxation, gibbs distributions, and the bayesian restoration of images. IEEE Transactions on Pattern Analysis and Machine Intelligence, PAMI-6(6):721-741, Nov 1984. ISSN 0162-8828. doi: 10.1109/TPAMI.1984. 4767596.

L. Gizon. LOI/SOHO constraints on oblique rotation of the solar core. In J. Provost and F.-X. Schmider, editors, Sounding Solar and Stellar Interiors: Poster Volume, volume 181 of IAU Symposium, 1996. 
L. Gizon and S. K. Solanki. Determining the Inclination of the Rotation Axis of a Sun-like Star. ApJ, 589:1009-1019, June 2003. doi: 10.1086/374715.

L. Gizon, J. Ballot, E. Michel, T. Stahn, G. Vauclair, H. Bruntt, P.-O. Quirion, O. Benomar, S. Vauclair, T. Appourchaux, M. Auvergne, A. Baglin, C. Barban, F. Baudin, M. Bazot, T. Campante, C. Catala, W. Chaplin, O. Creevey, S. Deheuvels, N. Dolez, Y. Elsworth, R. Garcia, P. Gaulme, S. Mathis, S. Mathur, B. Mosser, C. Regulo, I. Roxburgh, D. Salabert, R. Samadi, K. Sato, G. Verner, S. Hanasoge, and K. R. Sreenivasan. Seismic constraints on rotation of Sun-like star and mass of exoplanet. Proceedings of the National Academy of Science, 110:13267-13271, August 2013. doi: 10.1073/pnas.1303291110.

R. Glebocki and P. Gnacinski. VizieR Online Data Catalog: Catalog of Stellar Rotational Velocities (Glebocki+ 2005). VizieR Online Data Catalog, 3244:0, March 2005.

J. Goodman and J. Weare. Ensemble samplers with affine invariance. Communications in Applied Mathematics and Computational Science, 5:65-80, January 2010. doi: 10. 2140/camcos.2010.5.65.

D. F. Gray. The Observation and Analysis of Stellar Photospheres. September 2005.

M. R. Haas, N. M. Batalha, S. T. Bryson, D. A. Caldwell, J. L. Dotson, J. Hall, J. M. Jenkins, T. C. Klaus, D. G. Koch, J. Kolodziejczak, C. Middour, M. Smith, C. K. Sobeck, J. Stober, R. S. Thompson, and J. E. Van Cleve. Kepler Science Operations. ApJ, 713:L115-L119, April 2010. doi: 10.1088/2041-8205/713/2/L115.

R. Handberg and T. L. Campante. Bayesian peak-bagging of solar-like oscillators using MCMC: a comprehensive guide. AEFA, 527:A56, March 2011. doi: 10.1051/ 0004-6361/201015451.

J. D. Hartman, B. S. Gaudi, M. H. Pinsonneault, K. Z. Stanek, M. J. Holman, B. A. McLeod, S. Meibom, J. A. Barranco, and J. S. Kalirai. Deep MMT Transit Survey of the Open Cluster M37. III. Stellar Rotation at 550 Myr. ApJ, 691:342-364, January 2009. doi: 10.1088/0004-637X/691/1/342.

J. D. Hartman, G. Á. Bakos, G. Kovács, and R. W. Noyes. A large sample of photometric rotation periods for FGK Pleiades stars. MNRAS, 408:475-489, October 2010. doi: 10.1111/j.1365-2966.2010.17147.x.

L. Hartmann, R. Hewett, S. Stahler, and R. D. Mathieu. Rotational and radial velocities of T Tauri stars. ApJ, 309:275-293, October 1986. doi: 10.1086/164599.

J. Harvey. High-resolution helioseismology. In E. Rolfe and B. Battrick, editors, Future Missions in Solar, Heliospheric $\mathcal{E}$ Space Plasma Physics, volume 235 of ESA Special Publication, pages 199-208, June 1985.

J. W. Harvey, F. Hill, J. R. Kennedy, J. W. Leibacher, and W. C. Livingston. The Global Oscillation Network Group (GONG). Advances in Space Research, 8:117-120, 1988. doi: 10.1016/0273-1177(88)90304-3. 
W. K. Hastings. Monte carlo sampling methods using markov chains and their applications. Biometrika, 57:97-109, April 1970. doi: 10.2307/2334940. URL http: //www. jstor . org/stable/2334940.

C. B. Henderson and K. G. Stassun. Time-series Photometry of Stars in and around the Lagoon Nebula. I. Rotation Periods of 290 Low-mass Pre-main-sequence Stars in NGC 6530. ApJ, 747:51, March 2012. doi: 10.1088/0004-637X/747/1/51.

W. Herbst, J. F. Booth, D. L. Koret, G. V. Zajtseva, H. I. Shakhovskaya, F. J. Vrba, E. Covino, L. Terranegra, A. Vittone, D. Hoff, L. Kelsey, R. Lines, and W. Barksdale. Photometric variations of Orion population stars. V - A search for periodicities. AJ, 94 : 137-149, July 1987. doi: 10.1086/114456.

W. Herbst, C. A. L. Bailer-Jones, R. Mundt, K. Meisenheimer, and R. Wackermann. Stellar rotation and variability in the Orion Nebula Cluster. AEFA, 396:513-532, December 2002. doi: 10.1051/0004-6361:20021362.

W. Herbst, J. Eislöffel, R. Mundt, and A. Scholz. The Rotation of Young Low-Mass Stars and Brown Dwarfs. Protostars and Planets V, pages 297-311, 2007.

D. C. Hoaglin, F. Mosteller, and J. W. Tukey. Understanding Robust and Exploratory Data Analysis. Wiley, wiley clas edition, 2000. ISBN 978-0-471-38491-5.

E. Høg, C. Fabricius, V. V. Makarov, S. Urban, T. Corbin, G. Wycoff, U. Bastian, P. Schwekendiek, and A. Wicenec. The Tycho-2 catalogue of the 2.5 million brightest stars. AEFA, 355:L27-L30, March 2000.

R. Howard and J. Harvey. Spectroscopic Determinations of Solar Rotation. Sol. Phys., 12:23-51, April 1970. doi: 10.1007/BF02276562.

R. Howe. Solar Interior Rotation and its Variation. Living Reviews in Solar Physics, 6, February 2009. doi: 10.12942/lrsp-2009-1.

J. Irwin, S. Aigrain, S. Hodgkin, M. Irwin, J. Bouvier, C. Clarke, L. Hebb, and E. Moraux. The Monitor project: rotation of low-mass stars in the open cluster M34. MNRAS, 370: 954-974, August 2006. doi: 10.1111/j.1365-2966.2006.10521.x.

J. Irwin, S. Hodgkin, S. Aigrain, J. Bouvier, L. Hebb, M. Irwin, and E. Moraux. The Monitor project: rotation of low-mass stars in NGC 2362 - testing the disc regulation paradigm at 5 Myr. MNRAS, 384:675-686, February 2008a. doi: 10.1111/j.1365-2966. 2007.12725.x.

J. Irwin, S. Hodgkin, S. Aigrain, J. Bouvier, L. Hebb, and E. Moraux. The Monitor project: rotation of low-mass stars in the open cluster NGC 2547. MNRAS, 383:15881602, February 2008b. doi: 10.1111/j.1365-2966.2007.12669.x.

J. Irwin, S. Aigrain, J. Bouvier, L. Hebb, S. Hodgkin, M. Irwin, and E. Moraux. The Monitor project: rotation periods of low-mass stars in M50. MNRAS, 392:1456-1466, February 2009. doi: 10.1111/j.1365-2966.2008.14158.x. 
S. V. Jeffers, J.-F. Donati, E. Alecian, and S. C. Marsden. Observations of non-solar-type dynamo processes in stars with shallow convective zones. MNRAS, 411:1301-1312, February 2011. doi: 10.1111/j.1365-2966.2010.17762.x.

J. M. Jenkins, D. A. Caldwell, H. Chandrasekaran, J. D. Twicken, S. T. Bryson, E. V. Quintana, B. D. Clarke, J. Li, C. Allen, P. Tenenbaum, H. Wu, T. C. Klaus, C. K. Middour, M. T. Cote, S. McCauliff, F. R. Girouard, J. P. Gunter, B. Wohler, J. Sommers, J. R. Hall, A. K. Uddin, M. S. Wu, P. A. Bhavsar, J. Van Cleve, D. L. Pletcher, J. A. Dotson, M. R. Haas, R. L. Gilliland, D. G. Koch, and W. J. Borucki. Overview of the Kepler Science Processing Pipeline. ApJ, 713:L87-L91, April 2010. doi: 10.1088/ 2041-8205/713/2/L87.

S. Jester, D. P. Schneider, G. T. Richards, R. F. Green, M. Schmidt, P. B. Hall, M. A. Strauss, D. E. Vanden Berk, C. Stoughton, J. E. Gunn, J. Brinkmann, S. M. Kent, J. A. Smith, D. L. Tucker, and B. Yanny. The Sloan Digital Sky Survey View of the PalomarGreen Bright Quasar Survey. AJ, 130:873-895, September 2005. doi: 10.1086/432466.

T. Kallinger, B. Mosser, S. Hekker, D. Huber, D. Stello, S. Mathur, S. Basu, T. R. Bedding, W. J. Chaplin, J. De Ridder, Y. P. Elsworth, S. Frandsen, R. A. García, M. Gruberbauer, J. M. Matthews, W. J. Borucki, H. Bruntt, J. Christensen-Dalsgaard, R. L. Gilliland, H. Kjeldsen, and D. G. Koch. Asteroseismology of red giants from the first four months of Kepler data: Fundamental stellar parameters. AEFA, 522:A1, November 2010. doi: 10.1051/0004-6361/201015263.

T. Kallinger, J. De Ridder, S. Hekker, S. Mathur, B. Mosser, M. Gruberbauer, R. A. García, C. Karoff, and J. Ballot. The connection between stellar granulation and oscillation as seen by the Kepler mission. AEFA, 570:A41, October 2014. doi: 10.1051/0004-6361/201424313.

C. Karoff, T. S. Metcalfe, W. J. Chaplin, S. Frandsen, F. Grundahl, H. Kjeldsen, J. Christensen-Dalsgaard, M. B. Nielsen, S. Frimann, A. O. Thygesen, T. Arentoft, T. M. Amby, S. G. Sousa, and D. L. Buzasi. Sounding stellar cycles with Kepler - II. Ground-based observations. MNRAS, 433:3227-3238, August 2013. doi: $10.1093 / \mathrm{mnras} / \mathrm{stt} 964$.

S. D. Kawaler. Angular momentum loss in low-mass stars. ApJ, 333:236-247, October 1988. doi: 10.1086/166740.

Y.-C. Kim and P. Demarque. The Theoretical Calculation of the Rossby Number and the "Nonlocal" Convective Overturn Time for Pre-Main-Sequence and Early Post-MainSequence Stars. ApJ, 457:340, January 1996. doi: 10.1086/176733.

R. Kippenhahn, A. Weigert, and A. Weiss. Stellar Structure and Evolution. 2012. doi: 10.1007/978-3-642-30304-3.

H. Kjeldsen. PhD thesis, PhD thesis, University of Aarhus, Denmark, (1992), 1992.

H. Kjeldsen and T. R. Bedding. Amplitudes of stellar oscillations: the implications for asteroseismology. AEAA, 293:87-106, January 1995. 
H. Kjeldsen and T. R. Bedding. Amplitudes of solar-like oscillations: a new scaling relation. AEFA, 529:L8, May 2011. doi: 10.1051/0004-6361/201116789.

A. Koenigl. Disk accretion onto magnetic T Tauri stars. ApJ, 370:L39-L43, March 1991. doi: $10.1086 / 185972$.

R. P. Kraft. Stellar Rotation, page 385. 1970.

M. Küker and G. Rüdiger. Differential rotation of main sequence F stars. $A \mathcal{E} A, 433$ : 1023-1030, April 2005. doi: 10.1051/0004-6361:20041987.

D. W. Kurtz, H. Saio, M. Takata, H. Shibahashi, S. J. Murphy, and T. Sekii. Asteroseismic measurement of surface-to-core rotation in a main-sequence A star, KIC 11145123. MNRAS, 444:102-116, October 2014. doi: 10.1093/mnras/stu1329.

A. F. Lanza, M. L. Das Chagas, and J. R. De Medeiros. Measuring stellar differential rotation with high-precision space-borne photometry. AEEA, 564:A50, April 2014. doi: 10.1051/0004-6361/201323172.

T. P. Larson and J. Schou. Improved Helioseismic Analysis of Medium- $l$ Data from the Michelson Doppler Imager. Sol. Phys., 290:3221-3256, November 2015. doi: 10.1007/s11207-015-0792-y.

Y. Lebreton and M. J. Goupil. Asteroseismology for "à la carte" stellar age-dating and weighing. Age and mass of the CoRoT exoplanet host HD 52265. AE्EA, 569:A21, September 2014. doi: 10.1051/0004-6361/201423797.

N. R. Lomb. Least-squares frequency analysis of unequally spaced data. ApESSS, 39: 447-462, February 1976. doi: 10.1007/BF00648343.

M. Long, M. M. Romanova, and R. V. E. Lovelace. Locking of the Rotation of DiskAccreting Magnetized Stars. ApJ, 634:1214-1222, December 2005. doi: 10.1086/ 497000.

M. N. Lund, M. Lundkvist, V. Silva Aguirre, G. Houdek, L. Casagrande, V. Van Eylen, T. L. Campante, C. Karoff, H. Kjeldsen, S. Albrecht, W. J. Chaplin, M. B. Nielsen, P. Degroote, G. R. Davies, and R. Handberg. Asteroseismic inference on the spin-orbit misalignment and stellar parameters of HAT-P-7. AEAA, 570:A54, October 2014a. doi: 10.1051/0004-6361/201424326.

M. N. Lund, M. S. Miesch, and J. Christensen-Dalsgaard. Differential Rotation in Mainsequence Solar-like Stars: Qualitative Inference from Asteroseismic Data. ApJ, 790: 121, August 2014b. doi: 10.1088/0004-637X/790/2/121.

A. Maeder. Physics, Formation and Evolution of Rotating Stars. 2009. doi: 10.1007/ 978-3-540-76949-1.

A. Maeder and G. Meynet. The Evolution of Rotating Stars. ARAEFA, 38:143-190, 2000. doi: 10.1146/annurev.astro.38.1.143. 
A. Maeder and G. Meynet. Stellar evolution with rotation and magnetic fields. II. General equations for the transport by Tayler-Spruit dynamo. AEFA, 422:225-237, July 2004. doi: 10.1051/0004-6361:20034583.

E. E. Mamajek and L. A. Hillenbrand. Improved Age Estimation for Solar-Type Dwarfs Using Activity-Rotation Diagnostics. ApJ, 687:1264-1293, November 2008. doi: 10. 1086/591785.

J. P. Marques, M. J. Goupil, Y. Lebreton, S. Talon, A. Palacios, K. Belkacem, R.-M. Ouazzani, B. Mosser, A. Moya, P. Morel, B. Pichon, S. Mathis, J.-P. Zahn, S. TurckChièze, and P. A. P. Nghiem. Seismic diagnostics for transport of angular momentum in stars. I. Rotational splittings from the pre-main sequence to the red-giant branch. $A \mathcal{E} A$, 549:A74, January 2013. doi: 10.1051/0004-6361/201220211.

S. Mathur, T. S. Metcalfe, M. Woitaszek, H. Bruntt, G. A. Verner, J. ChristensenDalsgaard, O. L. Creevey, G. Doğan, S. Basu, C. Karoff, D. Stello, T. Appourchaux, T. L. Campante, W. J. Chaplin, R. A. García, T. R. Bedding, O. Benomar, A. Bonanno, S. Deheuvels, Y. Elsworth, P. Gaulme, J. A. Guzik, R. Handberg, S. Hekker, W. Herzberg, M. J. P. F. G. Monteiro, L. Piau, P.-O. Quirion, C. Régulo, M. Roth, D. Salabert, A. Serenelli, M. J. Thompson, R. Trampedach, T. R. White, J. Ballot, I. M. Brandão, J. Molenda-Żakowicz, H. Kjeldsen, J. D. Twicken, K. Uddin, and B. Wohler. A Uniform Asteroseismic Analysis of 22 Solar-type Stars Observed by Kepler. ApJ, 749:152, April 2012. doi: 10.1088/0004-637X/749/2/152.

G. Matijevič, A. Prša, J. A. Orosz, W. F. Welsh, S. Bloemen, and T. Barclay. Kepler Eclipsing Binary Stars. III. Classification of Kepler Eclipsing Binary Light Curves with Locally Linear Embedding. AJ, 143:123, May 2012. doi: 10.1088/0004-6256/143/5/ 123.

S. P. Matt, K. B. MacGregor, M. H. Pinsonneault, and T. P. Greene. Magnetic Braking Formulation for Sun-like Stars: Dependence on Dipole Field Strength and Rotation Rate. ApJ, 754:L26, August 2012. doi: 10.1088/2041-8205/754/2/L26.

A. Mazumdar, M. J. P. F. G. Monteiro, J. Ballot, H. M. Antia, S. Basu, G. Houdek, S. Mathur, M. S. Cunha, V. Silva Aguirre, R. A. García, D. Salabert, G. A. Verner, J. Christensen-Dalsgaard, T. S. Metcalfe, D. T. Sanderfer, S. E. Seader, J. C. Smith, and W. J. Chaplin. Measurement of Acoustic Glitches in Solar-type Stars from Oscillation Frequencies Observed by Kepler. ApJ, 782:18, February 2014. doi: 10.1088/0004-637X/782/1/18.

A. McQuillan, S. Aigrain, and T. Mazeh. Measuring the rotation period distribution of field M dwarfs with Kepler. MNRAS, 432:1203-1216, June 2013. doi: 10.1093/mnras/ stt536.

A. McQuillan, T. Mazeh, and S. Aigrain. Rotation Periods of 34,030 Kepler Mainsequence Stars: The Full Autocorrelation Sample. ApJS, 211:24, April 2014. doi: 10.1088/0067-0049/211/2/24. 
S. Meibom, R. D. Mathieu, and K. G. Stassun. Stellar Rotation in M35: Mass-Period Relations, Spin-Down Rates, and Gyrochronology. ApJ, 695:679-694, April 2009. doi: 10.1088/0004-637X/695/1/679.

S. Meibom, S. A. Barnes, D. W. Latham, N. Batalha, W. J. Borucki, D. G. Koch, G. Basri, L. M. Walkowicz, K. A. Janes, J. Jenkins, J. Van Cleve, M. R. Haas, S. T. Bryson, A. K. Dupree, G. Furesz, A. H. Szentgyorgyi, L. A. Buchhave, B. D. Clarke, J. D. Twicken, and E. V. Quintana. The Kepler Cluster Study: Stellar Rotation in NGC 6811. ApJ, 733:L9, May 2011. doi: 10.1088/2041-8205/733/1/L9.

S. Meibom, S. A. Barnes, I. Platais, R. L. Gilliland, D. W. Latham, and R. D. Mathieu. A spin-down clock for cool stars from observations of a 2.5-billion-year-old cluster. Nature, 517:589-591, January 2015. doi: 10.1038/nature14118.

L. Mestel. Magnetic braking by a stellar wind-I. MNRAS, 138:359, 1968. doi: 10.1093/ mnras/138.3.359.

L. Mestel. Angular Momentum Loss During Pre-Main Sequence Contraction. In S. L. Baliunas and L. Hartmann, editors, Cool Stars, Stellar Systems, and the Sun, volume 193 of Lecture Notes in Physics, Berlin Springer Verlag, page 49, 1984. doi: 10.1007/ 3-540-12907-3_179.

T. S. Metcalfe, O. L. Creevey, G. Doğan, S. Mathur, H. Xu, T. R. Bedding, W. J. Chaplin, J. Christensen-Dalsgaard, C. Karoff, R. Trampedach, O. Benomar, B. P. Brown, D. L. Buzasi, T. L. Campante, Z. Çelik, M. S. Cunha, G. R. Davies, S. Deheuvels, A. Derekas, M. P. Di Mauro, R. A. García, J. A. Guzik, R. Howe, K. B. MacGregor, A. Mazumdar, J. Montalbán, M. J. P. F. G. Monteiro, D. Salabert, A. Serenelli, D. Stello, Steşacute, M. licki, M. D. Suran, M. Yıldız, C. Aksoy, Y. Elsworth, M. Gruberbauer, D. B. Guenther, Y. Lebreton, K. Molaverdikhani, D. Pricopi, R. Simoniello, and T. R. White. Properties of 42 Solar-type Kepler Targets from the Asteroseismic Modeling Portal. ApJS, 214:27, October 2014. doi: 10.1088/0067-0049/214/2/27.

N. Metropolis, A. W. Rosenbluth, M. N. Rosenbluth, A. H. Teller, and E. Teller. Equation of state calculations by fast computing machines. The Journal of Chemical Physics, 21: 1087, December 1953. doi: 10.1063/1.1699114.

G. Meynet, P. Eggenberger, N. Mowlavi, and A. Maeder. Models for Pop I stars: implications for age determinations. In E. E. Mamajek, D. R. Soderblom, and R. F. G. Wyse, editors, The Ages of Stars, volume 258 of IAU Symposium, pages 177-188, June 2009. doi: $10.1017 / \mathrm{S} 1743921309031834$.

M. S. Miesch. Large-Scale Dynamics of the Convection Zone and Tachocline. Living Reviews in Solar Physics, 2, April 2005. doi: 10.12942/lrsp-2005-1.

J. Molenda-Żakowicz, S. G. Sousa, A. Frasca, K. Uytterhoeven, M. Briquet, H. Van Winckel, D. Drobek, E. Niemczura, P. Lampens, J. Lykke, S. Bloemen, J. F. Gameiro, C. Jean, D. Volpi, N. Gorlova, A. Mortier, M. Tsantaki, and G. Raskin. Atmospheric parameters of 169 F-, G-, K- and M-type stars in the Kepler field. MNRAS, 434:14221434, September 2013. doi: 10.1093/mnras/stt1095. 
E. Moraux, S. Artemenko, J. Bouvier, J. Irwin, M. Ibrahimov, T. Magakian, K. Grankin, E. Nikogossian, C. Cardoso, S. Hodgkin, S. Aigrain, and T. A. Movsessian. The Monitor Project: stellar rotation at $13 \mathrm{Myr}$. I. A photometric monitoring survey of the young open cluster h Persei. AEAA, 560:A13, December 2013. doi: 10.1051/0004-6361/ 201321508.

B. Mosser, F. Baudin, A. F. Lanza, J. C. Hulot, C. Catala, A. Baglin, and M. Auvergne. Short-lived spots in solar-like stars as observed by CoRoT. AEFA, 506:245-254, October 2009. doi: 10.1051/0004-6361/200911942.

B. Mosser, M. J. Goupil, K. Belkacem, J. P. Marques, P. G. Beck, S. Bloemen, J. De Ridder, C. Barban, S. Deheuvels, Y. Elsworth, S. Hekker, T. Kallinger, R. M. Ouazzani, M. Pinsonneault, R. Samadi, D. Stello, R. A. García, T. C. Klaus, J. Li, S. Mathur, and R. L. Morris. Spin down of the core rotation in red giants. AEFA, 548:A10, December 2012. doi: 10.1051/0004-6361/201220106.

J. A. Nelder and R. Mead. A simplex method for function minimization. The Computer Journal, 7(4):308-313, 1965. doi: 10.1093/comjnl/7.4.308. URL http: //comjnl. oxfordjournals .org/content/7/4/308.abstract.

M. B. Nielsen and C. Karoff. Starspot simulations for Kepler. Astronomische Nachrichten, 333:1036, December 2012. doi: 10.1002/asna.201211817.

M. B. Nielsen, L. Gizon, H. Schunker, and C. Karoff. Rotation periods of 12000 mainsequence Kepler stars: Dependence on stellar spectral type and comparison with v sin i observations. $A \mathcal{E} A$, 557:L10, September 2013. doi: 10.1051/0004-6361/201321912.

M. B. Nielsen, L. Gizon, H. Schunker, and J. Schou. Rotational splitting as a function of mode frequency for six Sun-like stars. AEFA, 568:L12, August 2014. doi: 10.1051/ 0004-6361/201424525.

A. A. Pamyatnykh, G. Handler, and W. A. Dziembowski. Asteroseismology of the $\beta$ Cephei star $v$ Eridani: interpretation and applications of the oscillation spectrum. $M N$ $R A S$, 350:1022-1028, May 2004. doi: 10.1111/j.1365-2966.2004.07721.x.

E. N. Parker. Dynamics of the Interplanetary Gas and Magnetic Fields. ApJ, 128:664, November 1958. doi: 10.1086/146579.

E. Paunzen, K. T. Wraight, L. Fossati, M. Netopil, G. J. White, and D. Bewsher. A photometric study of chemically peculiar stars with the STEREO satellites - II. Nonmagnetic chemically peculiar stars. MNRAS, 429:119-125, February 2013. doi: 10. 1093/mnras/sts318.

B. Paxton, L. Bildsten, A. Dotter, F. Herwig, P. Lesaffre, and F. Timmes. Modules for Experiments in Stellar Astrophysics (MESA). ApJS, 192:3, January 2011. doi: 10. 1088/0067-0049/192/1/3.

B. Paxton, M. Cantiello, P. Arras, L. Bildsten, E. F. Brown, A. Dotter, C. Mankovich, M. H. Montgomery, D. Stello, F. X. Timmes, and R. Townsend. Modules for Experiments in Stellar Astrophysics (MESA): Planets, Oscillations, Rotation, and Massive Stars. ApJS, 208:4, September 2013. doi: 10.1088/0067-0049/208/1/4. 
W. D. Pesnell, B. J. Thompson, and P. C. Chamberlin. The Solar Dynamics Observatory (SDO). Sol. Phys., 275:3-15, January 2012. doi: 10.1007/s11207-011-9841-3.

M. J. D. Powell. An efficient method for finding the minimum of a function of several variables without calculating derivatives. The Computer Journal, 7(2):155-162, 1964. doi: 10.1093/comjnl/7.2.155. URL http: //comjnl . oxfordjournals .org/ content/7/2/155. abstract.

P.-O. Quirion, J. Christensen-Dalsgaard, and T. Arentoft. Automatic Determination of Stellar Parameters Via Asteroseismology of Stochastically Oscillating Stars: Comparison with Direct Measurements. ApJ, 725:2176-2189, December 2010. doi: 10.1088/0004-637X/725/2/2176.

H. Rauer, C. Catala, C. Aerts, T. Appourchaux, W. Benz, A. Brandeker, J. ChristensenDalsgaard, M. Deleuil, L. Gizon, M.-J. Goupil, M. Güdel, E. Janot-Pacheco, M. MasHesse, I. Pagano, G. Piotto, D. Pollacco, C. Santos, A. Smith, J.-C. Suárez, R. Szabó, S. Udry, V. Adibekyan, Y. Alibert, J.-M. Almenara, P. Amaro-Seoane, M. A.-v. Eiff, M. Asplund, E. Antonello, S. Barnes, F. Baudin, K. Belkacem, M. Bergemann, G. Bihain, A. C. Birch, X. Bonfils, I. Boisse, A. S. Bonomo, F. Borsa, I. M. Brandão, E. Brocato, S. Brun, M. Burleigh, R. Burston, J. Cabrera, S. Cassisi, W. Chaplin, S. Charpinet, C. Chiappini, R. P. Church, S. Csizmadia, M. Cunha, M. Damasso, M. B. Davies, H. J. Deeg, R. F. Díaz, S. Dreizler, C. Dreyer, P. Eggenberger, D. Ehrenreich, P. Eigmüller, A. Erikson, R. Farmer, S. Feltzing, F. de Oliveira Fialho, P. Figueira, T. Forveille, M. Fridlund, R. A. García, P. Giommi, G. Giuffrida, M. Godolt, J. Gomes da Silva, T. Granzer, J. L. Grenfell, A. Grotsch-Noels, E. Günther, C. A. Haswell, A. P. Hatzes, G. Hébrard, S. Hekker, R. Helled, K. Heng, J. M. Jenkins, A. Johansen, M. L. Khodachenko, K. G. Kislyakova, W. Kley, U. Kolb, N. Krivova, F. Kupka, H. Lammer, A. F. Lanza, Y. Lebreton, D. Magrin, P. Marcos-Arenal, P. M. Marrese, J. P. Marques, J. Martins, S. Mathis, S. M, S. Messina, A. Miglio, J. Montalban, M. Montalto, M. J. P. F. G. Monteiro, H. Moradi, E. Moravveji, C. Mordasini, T. Morel, A. Mortier, V. Nascimbeni, R. P. Nelson, M. B. Nielsen, L. Noack, A. J. Norton, A. Ofir, M. Oshagh, R.-M. Ouazzani, P. Pápics, V. C. Parro, P. Petit, B. Plez, E. Poretti, A. Quirrenbach, R. Ragazzoni, G. Raimondo, M. Rainer, D. R. Reese, R. Redmer, S. Reffert, B. Rojas-Ayala, I. W. Roxburgh, S. Salmon, A. Santerne, J. Schneider, J. Schou, S. Schuh, H. Schunker, A. Silva-Valio, R. Silvotti, I. Skillen, I. Snellen, F. Sohl, S. G. Sousa, A. Sozzetti, D. Stello, K. G. Strassmeier, M. Švanda, G. M. Szabó, A. Tkachenko, D. Valencia, V. Van Grootel, S. D. Vauclair, P. Ventura, F. W. Wagner, N. A. Walton, J. Weingrill, S. C. Werner, P. J. Wheatley, and K. Zwintz. The PLATO 2.0 mission. Experimental Astronomy, 38:249-330, November 2014. doi: 10.1007/s10686-014-9383-4.

A. Reiners. Rotation- and temperature-dependence of stellar latitudinal differential rotation. AEFA, 446:267-277, January 2006. doi: 10.1051/0004-6361:20053911.

A. Reiners and S. Mohanty. Radius-dependent Angular Momentum Evolution in Lowmass Stars. I. ApJ, 746:43, February 2012. doi: 10.1088/0004-637X/746/1/43.

A. Reiners and J. H. M. M. Schmitt. On the feasibility of the detection of differential 
rotation in stellar absorption profiles. AEAA, 384:155-162, March 2002. doi: 10.1051/ 0004-6361:20011801.

A. Reiners, N. Joshi, and B. Goldman. A Catalog of Rotation and Activity in Early-M Stars. AJ, 143:93, April 2012. doi: 10.1088/0004-6256/143/4/93.

T. Reinhold and A. Reiners. Fast and reliable method for measuring stellar differential rotation from photometric data. AESA, 557:A11, September 2013. doi: 10.1051/ 0004-6361/201321161.

M. Rempel and R. Schlichenmaier. Sunspot modeling: From simplified models to radiative mhd simulations. Living Reviews in Solar Physics, 8(3), 2011. doi: 10.1007/lrsp-2011-3. URL http: //www . livingreviews . org/lrsp-2011-3.

F. Royer, J. Zorec, and A. E. Gómez. Rotational velocity distributions of A-type stars. In J. Zverko, J. Ziznovsky, S. J. Adelman, and W. W. Weiss, editors, The AStar Puzzle, volume 224 of IAU Symposium, pages 109-114, December 2004. doi: $10.1017 / \mathrm{S} 1743921304004442$.

A. E. Rydgren and F. J. Vrba. Periodic light variations in four pre-main-sequence K stars. ApJ, 267:191-198, April 1983. doi: 10.1086/160858.

A. Savitzky and M. J. E. Golay. Smoothing and differentiation of data by simplified least squares procedures. Analytical Chemistry, 36:1627-1639, 1964.

J. D. Scargle. Studies in astronomical time series analysis. II - Statistical aspects of spectral analysis of unevenly spaced data. ApJ, 263:835-853, December 1982. doi: $10.1086 / 160554$.

E. Schatzman. A theory of the role of magnetic activity during star formation. Annales d'Astrophysique, 25:18, February 1962.

J. Schou, H. M. Antia, S. Basu, R. S. Bogart, R. I. Bush, S. M. Chitre, J. ChristensenDalsgaard, M. P. Di Mauro, W. A. Dziembowski, A. Eff-Darwich, D. O. Gough, D. A. Haber, J. T. Hoeksema, R. Howe, S. G. Korzennik, A. G. Kosovichev, R. M. Larsen, F. P. Pijpers, P. H. Scherrer, T. Sekii, T. D. Tarbell, A. M. Title, M. J. Thompson, and J. Toomre. Helioseismic Studies of Differential Rotation in the Solar Envelope by the Solar Oscillations Investigation Using the Michelson Doppler Imager. ApJ, 505:390417, September 1998. doi: 10.1086/306146.

A. Skumanich. Time Scales for CA II Emission Decay, Rotational Braking, and Lithium Depletion. ApJ, 171:565, February 1972. doi: 10.1086/151310.

J. C. Smith, M. C. Stumpe, J. E. Van Cleve, J. M. Jenkins, T. S. Barclay, M. N. Fanelli, F. R. Girouard, J. J. Kolodziejczak, S. D. McCauliff, R. L. Morris, and J. D. Twicken. Kepler Presearch Data Conditioning II - A Bayesian Approach to Systematic Error Correction. PASP, 124:1000-1014, September 2012. doi: 10.1086/667697.

H. B. Snodgrass and R. K. Ulrich. Rotation of Doppler features in the solar photosphere. ApJ, 351:309-316, March 1990. doi: 10.1086/168467. 
S. K. Solanki. Sunspots: An overview. AEA Rev., 11:153-286, 2003. doi: 10.1007/ s00159-003-0018-4.

T. Stahn. Analysis of time series of solar-like oscillations - Applications to the Sun and HD 52265. PhD thesis, Göttingen University, Germany, 2010.

K. G. Strassmeier. Starspots. AEFA Rev., 17:251-308, September 2009. doi: 10.1007/ s00159-009-0020-6.

M. C. Stumpe, J. C. Smith, J. E. Van Cleve, J. D. Twicken, T. S. Barclay, M. N. Fanelli, F. R. Girouard, J. M. Jenkins, J. J. Kolodziejczak, S. D. McCauliff, and R. L. Morris. Kepler Presearch Data Conditioning I - Architecture and Algorithms for Error Correction in Kepler Light Curves. PASP, 124:985-999, September 2012. doi: $10.1086 / 667698$.

M. C. Stumpe, J. C. Smith, J. H. Catanzarite, J. E. Van Cleve, J. M. Jenkins, J. D. Twicken, and F. R. Girouard. Multiscale Systematic Error Correction via Wavelet-Based Bandsplitting in Kepler Data. PASP, 126:100-114, January 2014. doi: 10.1086/674989.

M. J. Thompson, J. Christensen-Dalsgaard, M. S. Miesch, and J. Toomre. The Internal Rotation of the Sun. ARAEEA, 41:599-643, 2003. doi: 10.1146/annurev.astro.41. 011802.094848 .

S. E. Thompson, J. L. Christiansen, J. M. Jenkins, D. A. Caldwell, T. Barclay, S. T. Bryson, C. J. Burke, J. R. Campbell, J. Catanzarite, B. D. Clarke, J. L. Coughlin, F. Girouard, M. R. Haas, K. Ibrahim, T. C. Klaus, J. J. Kolodziejczak, J. Li, S. D. McCauli, R. Morris, F. Mullally, E. V. Quintana, J. Rowe, A. Sabale, S. Seader, J. C. Smith, M. D. Still, P. G. Tenenbaum, J. D. Twicken, and A. K. Uddin. Kepler Data Release 21 Notes (KSCI-19061-001). Technical report, 2013.

S. A. Triana, E. Moravveji, P. I. Pápics, C. Aerts, S. D. Kawaler, and J. ChristensenDalsgaard. The Internal Rotation Profile of the B-type Star KIC 10526294 from Frequency Inversion of its Dipole Gravity Modes. ApJ, 810:16, September 2015. doi: 10.1088/0004-637X/810/1/16.

J. D. Twicken, H. Chandrasekaran, J. M. Jenkins, J. P. Gunter, F. Girouard, and T. C. Klaus. Presearch data conditioning in the Kepler Science Operations Center pipeline. In Society of Photo-Optical Instrumentation Engineers (SPIE) Conference Series, volume 7740 of Society of Photo-Optical Instrumentation Engineers (SPIE) Conference Series, page 77401U, July 2010. doi: 10.1117/12.856798.

V. Van Eylen, M. N. Lund, V. Silva Aguirre, T. Arentoft, H. Kjeldsen, S. Albrecht, W. J. Chaplin, H. Isaacson, M. G. Pedersen, J. Jessen-Hansen, B. Tingley, J. ChristensenDalsgaard, C. Aerts, T. L. Campante, and S. T. Bryson. What Asteroseismology can do for Exoplanets: Kepler-410A b is a Small Neptune around a Bright Star, in an Eccentric Orbit Consistent with Low Obliquity. ApJ, 782:14, February 2014. doi: 10.1088/0004-637X/782/1/14. 
J. L. van Saders, T. Ceillier, T. S. Metcalfe, V. S. Aguirre, M. H. Pinsonneault, R. A. García, S. Mathur, and G. R. Davies. Weakened magnetic braking as the origin of anomalously rapid rotation in old field stars. Nature, 529:181-184, January 2016. doi: 10.1038/nature16168.

A. H. Vaughan and G. W. Preston. A survey of chromospheric CA II H and K emission in field stars of the solar neighborhood. PASP, 92:385-391, August 1980. doi: 10.1086/ 130683.

H. Vázquez Ramió, T. Roca Cortés, and C. Régulo. Background solar irradiance spectrum at high and low phases of the solar activity cycle. In A. Wilson, editor, Solar Variability: From Core to Outer Frontiers, volume 506 of ESA Special Publication, pages 897-900, December 2002.

S. N. Vogel and L. V. Kuhi. Rotational velocities of pre-main-sequence stars. ApJ, 245: 960-976, May 1981. doi: 10.1086/158872.

H. von Zeipel. The radiative equilibrium of a rotating system of gaseous masses. MNRAS, 84:665-683, June 1924. doi: 10.1093/mnras/84.9.665.

L. M. Walkowicz and G. S. Basri. Rotation periods, variability properties and ages for Kepler exoplanet candidate host stars. MNRAS, 436:1883-1895, December 2013. doi: 10.1093/mnras/stt1700.

E. J. Weber and L. Davis, Jr. The Angular Momentum of the Solar Wind. ApJ, 148: 217-227, April 1967. doi: 10.1086/149138.

O. C. Wilson. Flux Measurements at the Centers of Stellar h- and K-Lines. ApJ, 153:221, July 1968. doi: 10.1086/149652.

Brian E. Wood. Astrospheres and solar-like stellar winds. Living Reviews in Solar Physics, 1(2), 2004. doi: 10.1007/lrsp-2004-2. URL http: //www . livingreviews . org/lrsp-2004-2.

M. F. Woodard. Short-Period Oscillations in the Total Solar Irradiance. PhD thesis, UNIVERSITY OF CALIFORNIA, SAN DIEGO., 1984.

K. T. Wraight, L. Fossati, M. Netopil, E. Paunzen, M. Rode-Paunzen, D. Bewsher, A. J. Norton, and G. J. White. A photometric study of chemically peculiar stars with the STEREO satellites - I. Magnetic chemically peculiar stars. MNRAS, 420:757-772, February 2012. doi: 10.1111/j.1365-2966.2011.20090.x. 
Appendix 



\section{A Clusters used in Fig. 1.1}

Table A.1: Clusters and their ages used in Fig. 1.1.

\begin{tabular}{lccl}
\hline Cluster & Age $[\mathrm{Myr}]$ & Mean $P_{\text {rot }}$ & Reference \\
\hline ONC & 1. & 3.94 & Herbst et al. (2002) \\
NGC6530 & 1.65 & 5.04 & Henderson and Stassun (2012) \\
NGC2264 & 2 & 2.57 & Affer et al. (2013) \\
NGC2362 & 5 & 1.30 & Irwin et al. (2008a) \\
NGC869 & 13 & 2.58 & Moraux et al. (2013) \\
NGC2547 & 40 & 4.04 & Irwin et al. (2008b) \\
M50 & 62 & 2.21 & Irwin et al. (2009) \\
Pleiades & 125 & 3.02 & Hartman et al. (2010) \\
M35 & 150 & 3.59 & Meibom et al. (2009) \\
M34 & 250 & 4.46 & Irwin et al. (2006) \\
M48 & 450 & 7.51 & Barnes et al. (2015) \\
M37 & 550 & 7.53 & Hartman et al. (2009) \\
Hyades & 600 & 11.72 & Delorme et al. (2011) \\
NGC6811 & 1000 & 10.37 & Meibom et al. (2011) \\
NGC6819 & 2500 & 18.25 & Meibom et al. (2015) \\
\hline
\end{tabular}





\section{$B$ Detrending and corrections in PDC/msMAP data}

A significant long-term trend that spans the entire length of the time series can be thought of as an unresolved oscillation. The effect in the power spectrum is a high level of red noise, stemming from what amounts to the window function of a peak with a frequency less than the minimum frequency of $1 / T$.

Depending on the purpose of the analysis, one may wish to remove the long term trends. If the cause of the trends is known and well understood, where ancillary data shows the exact cause of the variability, it may be removed based on this information. On the other hand when the nature of the variability is not clear one must apply an empirical correction method.

Typical effects in the time series may include smooth variations of the measured quantity or discontinuous jumps. The latter can be easily corrected by shifting the data, provided it is clear that the jumps are not intrinsic to the star. The former is more difficult to treat, since there may be a smooth transition in both frequency and amplitude between the instrumental variability and the intrinsic stellar variability. Typically a frequency limit is set, below which variability is removed either completely or gradually. An example is fitting polynomials to sections of the time series, the order of the polynomial and the sections that are fit to define the lower frequency limit ${ }^{1}$

When considering the data products from the Kepler mission, one must remember the overall mission statement which is to measure the frequency of exoplanets around other stars. Any correction and treatment of the produced light curves have therefore been optimized for detecting exoplanets around a large number of very different stars, an optimization that may not necessarily coincide with other analyses.

The number of systematic sources of noise in the raw Kepler data is quite extensive (Christiansen et al. 2013). The most prominent are the perhaps the jumps between each observing period of three months (quarter). These occur when the satellite rotates to accommodate the change of position in its orbit and the direction of Earth for downloading data, and so the stars land on different CCDs with slightly different flux response functions. Secondly these rolls also induce temperature changes in the spacecraft, thereby changing the focus of the telescope (Haas et al. 2010). This causes an overall broadening of a stars point spread function (PSF). Since the aperture for each star is fixed at the beginning of each quarter (in post-processing), a broadening of the PSF will cause less overall light to fall on the aperture. When the spacecraft has reached thermal equilibrium once more the PSF returns to normal. Similarly if there is a pointing drift of the space-

\footnotetext{
${ }^{1}$ This is the basic premise of Savitzky-Golay filtering (Savitzky and Golay 1964).
} 
craft during the observations, some of the pixels will receive less light, also leading to an overall drop in measured flux.

The PDC (Pre-search Data Conditioning) pipeline is applied before public release of the data to correct these systematic variations. The details of the pipeline have evolved considerably over the Kepler mission lifetime, but for the most part have revolved around the concept of cotrending (Twicken et al. 2010, Jenkins et al. 2010, Stumpe et al. 2012, Smith et al. 2012). The pipeline attempts to identify variability that is common to a subset of otherwise quiet stars, and then remove this variability from the entirety of the observed sample. The idea is that common variability seen in a sample of quiet stars would represent that induced by the spacecraft and instrumentation, and not be intrinsic stellar variability.

While this functions well for the objective of detecting planetary transits, it also strongly reduces the amplitude of variability with periods greater than $\sim 20$ day periods, and almost completely removed variability at periods longer than 30 days. For particularly active dwarf stars where the variability amplitude is high, like the majority of the stars analyzed in Chapter 2, this pipeline does not necessarily pose a problem. However, for less active stars like those studied in chapters 3 and 4 more care must be used when studying periods on long time scales. 


\section{Measuring rotation with spectroscopy}

For completeness in relation to Chapter 2, a brief description of the measurement of the projected rotational velocity $v \sin i$ is provided here.

The motion of plasma in the photosphere of a star is a superposition of various velocities. These velocities stem from a variety of sources like simple thermal motion, convective up-welling, turbulence, as well as rotation. The overall effect of a velocity field on the stellar surface is to Doppler shift the light emitted from the photosphere along the line of sight to the observer. This broadens any line profiles in a spectrogram taken from the star.

The velocity fields are not all isotropic, i.e., the granulation motion is predominantly radial and horizontal at the surface, while rotation velocity is azimuthal. The corresponding Doppler shifts therefore have different effects on the line profiles. This results in a total line profile consisting of a convolution of broadening profiles from the different effects.

For a rapidly rotating star (tens of $\mathrm{km} / \mathrm{s}$ or more) the line profiles are dominated by rotational broadening. In such a case the full width at half maximum of the line is a good rough estimate of the projected rotation rate $v \sin i$, where $i$ is the angle of the stellar rotation axis relative to the line of sight to the observer (Gray 2005). For slower rotators like the Sun however, the rotational velocity becomes comparable to other motions like turbulent convection. This places more stringent requirements on instrumentation, and typically results in large relative uncertainties. A model is then usually fit to the spectrum, or it is compared to synthetic template spectra.

Using the spectroscopic $v \sin i$ to estimate surface velocities has the obvious benefit of only having to measure a single spectrogram of the star. This may be done for tens of stars during a night, and so building catalogs of hundreds or thousands of stars is relatively easy and inexpensive. The main problem however, lies in that the measured velocities are only the two-dimensional projections of the true three-dimensional velocities. To determine the rotational velocity of the star the inclination of the rotation axis must be used, which is often difficult to obtain. 



\section{Publications}

\section{Refereed publications}

- H. Schunker, J. Schou, W. H. Ball, M. B. Nielsen, and L. Gizon. Asteroseismic inversions for radial differential rotation of Sun-like stars: ensemble fits. A\&A, 586:A79, February 2016. doi: 10.1051/0004-6361/201527485.

- M. B. Nielsen, L. Gizon, H. Schunker, and C. Karoff. Rotation periods of 12000 main sequence Kepler stars: Dependence on stellar spectral type and comparison with v sin i observations. A\&A, 557:L10, September 2013. doi: 10.1051/0004$6361 / 201321912$.

- M. B. Nielsen, L. Gizon, H. Schunker, and J. Schou. Rotational splitting as a function of mode frequency for six Sun-like stars. A\&A, 568:L12, August 2014. doi: 10.1051/ 0004-6361/201424525.

- M. B. Nielsen, H. Schunker, L. Gizon, and W. H. Ball. Constraining dierential rotation of Sun-like stars from asteroseismic and starspot rotation periods. A\&A, 582:A10, October 2015. doi: 10.1051/0004-6361/201526615.

- M. N. Lund, M. Lundkvist, V. Silva Aguirre, G. Houdek, L. Casagrande, V. Van Eylen, T. L. Campante, C. Karoff, H. Kjeldsen, S. Albrecht, W. J. Chaplin, M. B. Nielsen, P. Degroote, G. R. Davies, and R. Handberg. Asteroseismic inference on the spin-orbit misalignment and stellar parameters of HAT-P-7. A\&A, 570:A54, October 2014b. doi: 10.1051/0004-6361/201424326.

- H. Rauer, C. Catala, C. Aerts, T. Appourchaux, W. Benz, A. Brandeker, J. ChristensenDalsgaard, M. Deleuil, L. Gizon, M.-J. Goupil, M. Güdel, E. Janot-Pacheco, M. Mas- Hesse, I. Pagano, G. Piotto, D. Pollacco, C . Santos, A. Smith, J.-C. Suárez, R. Szabó, S. Udry, V. Adibekyan, Y. Alibert, J.-M. Almenara, P. Amaro-Seoane, M. A.-v. Ei, M. Asplund, E. Antonello, S. Barnes, F. Baudin, K. Belkacem, M. Bergemann, G. Bihain, A. C. Birch, X. Bonfils, I. Boisse, A. S. Bonomo, F. Borsa, I. M. Brandăo, E. Brocato, S. Brun, M. Burleigh, R. Burston, J. Cabrera, S. Cassisi,W. Chaplin, S. Charpinet, C. Chiappini, R. P. Church, S. Csizmadia, M. Cunha, M. Damasso, M. B. Davies, H. J. Deeg, R. F. Díaz, S. Dreizler, C. Dreyer, P. Eggenberger, D. Ehrenreich, P. Eigmüller, A. Erikson, R. Farmer, S. Feltzing, F. de Oliveira Fialho, P. Figueira, T. Forveille, M. Fridlund, R. A. García, P. Giommi, G. Giurida, M. Godolt, J. Gomes da Silva, T. Granzer, J. L. Grenfell, A. GrotschNoels, E. Günther, C. A. Haswell, A. P. Hatzes, G. Hébrard, S. Hekker, R. Helled, 
K. Heng, J. M. Jenkins, A. Johansen, M. L. Khodachenko, K. G. Kislyakova,W. Kley, U. Kolb, N. Krivova, F. Kupka, H. Lammer, A. F. Lanza, Y. Lebreton, D. Magrin, P. Marcos-Arenal, P. M. Marrese, J. P. Marques, J. Martins, S. Mathis, S. M, S. Messina, A. Miglio, J. Montalban, M. Montalto, M. J. P. F. G. Monteiro, H. Moradi, E. Moravveji, C. Mordasini, T. Morel, A. Mortier, V. Nascimbeni, R. P. Nelson, M. B. Nielsen, L. Noack, A. J. Norton, A. Ofir, M. Oshagh, R.-M. Ouazzani, P. Pápics, V. C. Parro, P. Petit, B. Plez, E. Poretti, A. Quirrenbach, R. Ragazzoni, G. Raimondo, M. Rainer, D. R. Reese, R. Redmer, S. Reert, B. Rojas-Ayala, I. W. Roxburgh, S. Salmon, A. Santerne, J. Schneider, J. Schou, S. Schuh, H. Schunker, A. Silva-Valio, R. Silvotti, I. Skillen, I. Snellen, F. Sohl, S. G. Sousa, A. Sozzetti, D. Stello, K. G. Strassmeier, M. Švanda, G. M. Szabó, A. Tkachenko, D. Valencia, V. Van Grootel, S. D. Vauclair, P. Ventura, F. W. Wagner, N. A. Walton, J. Weingrill, S. C. Werner, P. J. Wheatley, and K. Zwintz. The PLATO 2.0 mission. Experimental Astronomy, 38:249-330, November 2014. doi: 10.1007/s10686-014-9383-4.

- R. A. García, T. Ceillier, D. Salabert, S. Mathur, J. L. van Saders, M. Pinsonneault, J. Ballot, P. G. Beck, S. Bloemen, T. L. Campante, G. R. Davies, J.-D. do Nascimento, Jr., . Mathis, T. S. Metcalfe, M. B. Nielsen, J. C. SuÃąrez, W. J. Chaplin, A. Jiménez, and C. Karoff. Rotation and magnetism of Kepler pulsating solar-like stars. Towards asteroseismically calibrated age-rotation relations. A\&A, 572:A34, December 2014. doi: 10.1051/0004-6361/201423888.

- S. Aigrain, J. Llama, T. Ceillier, M. L. d. Chagas, J. R. A. Davenport, R. A. García, K. L. Hay, A. F. Lanza, A. McQuillan, T. Mazeh, J. R. de Medeiros, M. B. Nielsen, and T. Reinhold. Testing the recovery of stellar rotation signals from Kepler light curves using a blind hare-and-hounds exercise. MNRAS, 450:3211-3226, July 2015. doi: 10.1093/mnras/stv853.

- C. Karoff, T. S. Metcalfe, W. J. Chaplin, S. Frandsen, F. Grundahl, H. Kjeldsen, J. Christensen-Dalsgaard, M. B. Nielsen, S. Frimann, A. O. Thygesen, T. Arentoft, T. M. Amby, S. G. Sousa, and D. L. Buzasi. Sounding stellar cycles with Kepler - II. Ground-based observations. MNRAS, 433:3227-3238, August 2013. doi: 10.1093/mnras/stt964.

\section{Conference proceedings}

- M. B. Nielsen, L. Gizon, H. Schunker, and C. Karo. Measuring Stellar Rotation Periods with Kepler. In H. Shibahashi and A. E. Lynas-Gray, editors, Progress in Physics of the Sun and Stars: A New Era in Helio- and Asteroseismology, volume 479 of Astronomical Society of the Pacific Conference Series, page 137, December $2013 b$. 


\section{Acknowledgements}

I would like to especially thank my supervisors Laurent and Hannah. Thank you Laurent, for keeping me on track and seeing the bigger picture during this project and the writing of this thesis. Thank you Hannah, for listening to and sorting through all my sometimes not quite simply posed and often random questions.

There are of course also a multitude of people who were not directly related to this project, but still provided much advice and many answers to my questions when other help was not available. Thank you Jesper, for always having an answer to my most obscure and detailed questions. Thank you Warrick, for my often repeated and sudden interruptions of your work with questions that were likely quite trivial (and also the philosocoffee). Thank you Timo for all the useful discussions and for being an excellent office-mate.

I have also found many friends during my $\mathrm{PhD}$, thank you all for listening to my complaints and worries. Thank you Jan, thank you Björn, for helping me find my way through the forest of paperwork and translation that is associated with doing a $\mathrm{PhD}$ in Germany. Thank you coffee group, for all the chocolate.

This thesis project was in part funded by the Deutscher Akademischer Austauschdienst (DAAD) through the Go8 Australia-Germany Joint Research Co-operation Scheme. I also acknowledge research funding by Deutsche Forschungsgemeinschaft (DFG) under grant SFB 963/1 “Astrophysical flow instabilities and turbulence" (Project A18).

Lastly, I would also like to thank the members of my thesis defense committee, for taking the time read this long document and for attending my defense. 



\title{
Curriculum vitae
}

\author{
Martin Bo Nielsen
}

Born: 19.11.1985 Nationality: Danish

Institut für Astrophysik,

Georg-August-Universität Göttingen

Friedrich-Hund-Platz 1, 37077 Göttingen

\section{Main topics}

Helioseismology and asteroseismology

Activity and rotation of Sun-like stars
Phone: +49 (0)551 3914156

Email: nielsen@mps.mpg.de

Kepler data analysis

\section{Education}

2012-2016: PhD Student, Institute for Astrophysics Göttingen and Max Planck Institute For Solar System Research, Germany

Title: Differential rotation of Sun-like stars from starspots and asteroseismology Supervisors: Prof. L. Gizon, Dr. H. Schunker and Prof. A. Reiners

Successfully defended with Summa Cum Laude on Apr. 202016

2009-2012: Master of Science, Aarhus University, Denmark (conferred: May 8 2012, highest marks)

Title: "Photometric observations of star spots"

Supervisors: Prof. H. Kjeldsen and Dr. C. Karoff

2006-2009: Bachelor of Science, Aarhus University, Denmark (conferred: Apr. 19 2010, highest marks)

Major: Astronomy and Astrophysics

Title: "A numerical investigation of the properties

of Lagrange points in the restricted three-body problem"

Supervisor: Prof. H. Kjeldsen

\section{Publications}

Refereed Papers:

1. "Constraining differential rotation of Sun-like stars from asteroseismic and starspot rotation periods" - M. B. Nielsen, L. Gizon, H. Schunker, W. H. Ball. 2015 A\&A, 582, A10.

2. "Rotational splitting as a function of mode frequency for six Sun-like stars" - M. B. Nielsen, L. Gizon, H. Schunker, J. Schou. 2014, A\&A, 568, L12.

3. "Rotation periods of 12000 main-sequence Kepler stars" - M. B. Nielsen, L. Gizon, H. Schunker, C. Karoff. 2013, A\&A, 557, L10. 
Co-authored refereed Papers:

1. H. Schunker \& 4 co-authors incl. M. B. Nielsen. 2016, A\&A, 586, A79.

2. S. Aigrain \& 12 co-authors incl. M. B. Nielsen. 2015, MNRAS, 450, 3211-3226.

3. R. Garcia \& 18 co-authors incl. M. B. Nielsen. 2014, A\&A, 572, A34.

4. M. Lund \& 14 co-authors incl. M. B. Nielsen. 2014, A\&A, 570, A54..

5. H. Rauer \& 159 co-authors incl. M. B. Nielsen. 2014, Exp. Astron. 38, 249-330.

6. C. Karoff \& 12 co-authors incl. M. B. Nielsen. 2013, MNRAS, 433, 3227.

7. J. P. Merrison \& 11 co-authors incl. M. B. Nielsen. 2012, P\&SS, 60, 328.

8. A. S. Baran \& 3 co-authors incl. M. B. Nielsen. 2011, Acta Astron., 61, 325.

Additional papers:

1. Kepler White Paper: Asteroseismology of Solar-Like Oscillators in a 2-Wheel Mission

W. J. Chaplin \& 56 co-authors incl. M. B. Nielsen. 2013, ArXiv:1309.0702.

2. M. B. Nielsen \& C. Karoff. 2012, AN, 333, 1036.

3. M. B. Nielsen, L. Gizon, H. Schunker, C. Karoff. 2013, ASPC, 479, 137.

\section{Presentations}

Contributed Talks:

- "Differential rotation in Sun-like stars: Comparison of rotation from asteroseismology and surface activity" KASC8/TASC1 Conference, Jun. 182015 Aarhus, Denmark

- "Constraints on the radial differential rotation of six Sun-like stars" Plato 2.0 Science Conference, Dec. 5 2014, Taormina, Italy.

- "Measuring rotation from star spots in the Kepler field" SpaceInn Workshop 4.1 Peak-bagging in Helio- and Asteroseismology, Mar. 12 2014, Tenerife, Spain.

Seminars:

- "Constraining radial differential rotation in Sun-like stars"

Presented at Sydney Institute for Astronomy, Nov. 27 , 2015, Sydney, Australia; and at the Solar System School Seminar, Dec. 16 2015, Max Planck Institute for Solar System Research, Göttingen, Germany.

- "Asteroseismic determination of internal rotation in Sun-like stars" Solar System School Seminar, Nov. 5 2014, Max Planck Institute for Solar System Research, Göttingen, Germany.

- "Measuring stellar rotation in the Kepler field" CRC Colloquium Talk, Jun. 4 2014, Institute for Astrophysics, Göttingen, Germany. 
- "Stellar rotation from starspot variability" Solar System School Seminar, Aug. 19 2013, Max Planck Institute for Solar System Research, Göttingen, Germany.

Poster Presentations:

- "Rotational splitting as a function of frequency in six Sun-like stars" HELAS 6 meeting, Sep. 2014, Göttingen, Germany.

- "Stellar rotation from starspot variability" Second Kepler Science Conference, Nov. 2013, NASA Ames, USA.

- "Searching for Stellar Rotation with Kepler"

Fachbeirat evaluation, 2013, Max Planck Institute for Solar System Research, Göttingen, Germany.

- "A study of stellar rotation from star spot signals in the Kepler field"

Fifth KASC Workshop, Balatonalmádi, Hungary

Annual Danish Astronomy meeting, 2012, Hven, Denmark

The Modern Era of Helio- Asteroseismology ESF Conference, 2012, Obergurgl, Austria.

- "Revealing the roots of stellar cycles"

Fourth KASC Workshop, Jul. 2011, Boulder, USA.

- "The SONG about the stars"

Third KASC Workshop, Jun. 2010, Aarhus, Denmark.

\section{Teaching and Outreach}

2016: Research featured in the Max Planck Gesellschaft Year Book

2015: Lab Course in Observational Astronomy Methods (Course planning and tutoring)

2013-2014: Data Analysis in Astrophysics (Teaching assistant and lecturing)

\section{International Research Visits}

2015: 1 Month at Sydney University, under grant by the German Academic Exchange Service (DAAD)

\section{Schools}

- Nordic Optical Telescope Winter School 2011

- Wroclaw Spectroscopy school 2013

- Aarhus Spectroscopy school 2014 


\section{Research Related Positions:}

2011-2012: Student Support Astronomer at Nordic Optical Telescope,

Santa Cruz de La Palma, Spain

2009-2011: Accelerator Operator at AMS Carbon-14 Dating Centre, Aarhus University, Denmark

\section{Skills}

Programming Languages

- Python (Astropy, EMCEE, Numpy, Scipy, Multiprocessing, f2py)

- Matlab (wrote a simple stellar structure modeling code as course exam project)

- Fortran (basic use of equations to speed up computations in Python)

Languages

- Danish: Native

- English: Excellent (written and verbal)

- German: Basic (Entry level course taken in 2014)

Other

- Basic first aid

\section{Contacts}

Prof. Laurent Gizon (PhD supervisor)

Director of the Max Planck Institute for Solar System Research

Justus-von-Liebig-Weg 3, 37077, Göttingen, Germany

Phone:+49 551384979439

Fax:+49 551384979240

Email: gizon@mps.mpg.de

Prof. Hans Kjeldsen (Masters and Bachelors supervisor)

Professor of astrophysics at the Department of Astrophysics at Aarhus University

Ny Munkegade 120, building 1520, room 527, 8000 Aarhus C, Denmark

Phone:+458715 5687

Email: hans@phys.au.dk

Dr. Hannah Schunker (PhD thesis advisory committee member)

Researcher at Max Planck Institute for Solar System Research

Justus-von-Liebig-Weg 3, 37077, Göttingen, Germany

Phone: +49551384979440

Email: schunker@mps.mpg.de 
Prof. Angar Reiners (PhD thesis advisory committee member) Professor of astrophysics at Institut für Astrophysik, Universität Göttingen Friedrich-Hund-Platz 1, D-37077 Göttingen

Phone: +49 5513913825

Email: Ansgar.Reiners@phys.uni-goettingen.de 